Louisiana State University

LSU Digital Commons

1998

\title{
An Examination of Automatic Versus Strategic Semantic Priming Effects in Broca's Aphasia.
}

Janice Feagin Del toro

Louisiana State University and Agricultural \& Mechanical College

Follow this and additional works at: https://digitalcommons.Isu.edu/gradschool_disstheses

\section{Recommended Citation}

Del toro, Janice Feagin, "An Examination of Automatic Versus Strategic Semantic Priming Effects in Broca's Aphasia." (1998). LSU Historical Dissertations and Theses. 6823.

https://digitalcommons.Isu.edu/gradschool_disstheses/6823

This Dissertation is brought to you for free and open access by the Graduate School at LSU Digital Commons. It has been accepted for inclusion in LSU Historical Dissertations and Theses by an authorized administrator of LSU Digital Commons. For more information, please contact gradetd@lsu.edu. 


\section{INFORMATION TO USERS}

This manuscript has been reproduced from the microfilm master. UMI films the text directly from the original or copy submitted. Thus, some thesis and dissertation copies are in typewriter face, while others may be from any type of computer printer.

The quality of this reproduction is dependent upon the quality of the copy submitted. Broken or indistinct print, colored or poor quality illustrations and photographs, print bleedthrough, substandard margins, and improper alignment can adversely affect reproduction.

In the unlikely event that the author did not send UMI a complete manuscript and there are missing pages, these will be noted. Also, if unauthorized copyright material had to be removed, a note will indicate the deletion.

Oversize materials (e.g., maps, drawings, charts) are reproduced by sectioning the original, beginning at the upper left-hand corner and continuing from left to right in equal sections with small overlaps. Each original is also photographed in one exposure and is included in reduced form at the back of the book.

Photographs included in the original manuscript have been reproduced xerographically in this copy. Higher quality 6" $\times 9$ " black and white photographic prints are available for any photographs or illustrations appearing in this copy for an additional charge. Contact UMI directly to order.

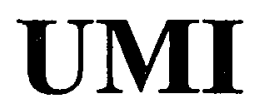

A Bell \& Howell Information Company 300 North Zeeb Road, Ann Arbor MI 48106-1346 USA

$313 / 761-4700 \quad 800 / 521-0600$ 


\section{Reproduced with permission of the copyright owner. Further reproduction prohibited without permission.}




\title{
AN EXAMINATION OF AUTOMATIC VERSUS STRATEGIC SEMANTIC PRIMING EFFECTS IN BROCA'S APHASIA
}

\author{
A Dissertation \\ Submitted to the Graduate Faculty of the \\ Louisiana State University and \\ Agricultural and Mechanical College \\ in partial fulfillment of the \\ requirements for the degree of \\ Doctor of Philosophy \\ in \\ Communication Sciences and Disorders
}

by

Janice Feagin Del Toro

B.A., University of Florida, 1981

M.A., University of Florida, 1987

December, 1998 
UMI Number: 9922072

UMI Microform 9922072

Copyright 1999, by UMI Company. All rights reserved.

This microform edition is protected against unauthorized copying under Title 17, United States Code.

\section{UMI}

300 North Zeeb Road

Ann Arbor, MI 48103

Reproduced with permission of the copyright owner. Further reproduction prohibited without permission. 


\section{ACKNOWLEDGEMENTS}

In my own stubborn and independent way, I chose to pursue a dissertation research topic unfamiliar to most of my committee members. I would like to thank my major professor, Dr. Hugh Buckingham, for allowing me the freedom to do so, and for supporting my venture by reading and discussing with me critical pieces of the literature I needed to understand in order to formulate the hypotheses that guided my experiments. I would also like to thank Dr. Janet McDonald for agreeing to serve as my co-chair, and for providing me with the guidance that I needed to bring the project to its completion. I am grateful for the remaining members of my committee, Dr. Paul Hoffman, Dr. Janna Oetting, and Dr. Drew Gouvier for their encouragement, and for patiently allowing me to teach them something about semantic priming.

I am indebted to the College of Arts and Sciences and to the faculty of the Department of Communication Sciences and Disorders for offering me an instructorship, and the concomitant funds needed to set up my laboratory. I would like to express my gratitude to Dr. Kenneth Forster of the University of Arizona for assisting me in selecting the hardware and software I needed to perform priming experiments, and for his patient guidance with programming the specific files needed for my dissertation work. Setting up my lab was no easy task, and Dr. Lee Mendoza helped me with numerous technological quandaries. I thank him for helping me past a myriad of seemingly impassable hurdles and for his ongoing moral support. I would also like to acknowledge Stephanie Daniels of the Veteran's Administration Medical Center in New Orleans, Susan Graham, of Our Lady of the Lake Regional Medical Center in Baton 
Rouge, and Jeanne Fisher of the Louisiana State University Speech and Hearing Clinic in Baton Rouge for their assistance in helping me locate subjects with Broca's aphasia. I appreciate all the wonderful encouragement I have received from the students and faculty of the Department of Communication Sciences and Disorders. I feel very fortunate to work with such a terrific group of people. A special thanks goes out to those wonderfully intelligent, witty, and fun women of the Dean French House -- aren't we lucky to have each other as friends? Finally, I would like to thank my family for all of their love and encouragement as I have faced the challenges of the past two years. They have taught me I really don't have to go it alone. 


\section{TABLE OF CONTENTS}

Acknowledgments........................................................................................ ii

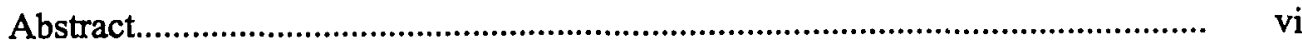

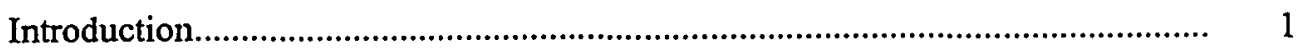

End Notes.............................................................................................. 5

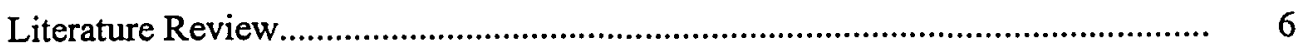

Semantic Priming .................................................................................... 6

Automatic Priming Effects ......................................................... 7

Strategic Priming Effects .......................................................... 10

Expectancy ................................................................. 11

Postlexical Priming Mechanisms .................................. 15

The Neely and Keefe Three Process Theory of Priming........................... $\quad 21$

Semantic Priming in Broca's Aphasia.......................................................... $\quad 30$

End Notes......................................................................................... 41

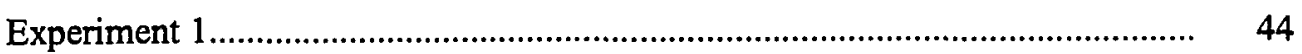

Methods.......................................................................................... 45

Stimulus Materials and List Construction ................................. 45

Procedure......................................................................... 50

Subjects.......................................................................... 52

Results............................................................................................. 53

Word Target Data................................................................... 56

Reaction Time Analysis............................................... 56

Error Analysis................................................................ 67

Nonword Target Data............................................................... 75

Reaction Time Analysis................................................ 75

Error Analysis............................................................ 79

Discussion............................................................................................ 81

Word Target Data...................................................................... 81

Nonword Target Data............................................................... 84

End Notes........................................................................................ 85

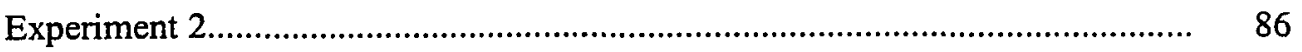

Pilot Study 1............................................................................... 86

Methods.......................................................................... 87

Design........................................................................ 87

Subjects..................................................................... 87

Stimuli and Procedures................................................... 88 


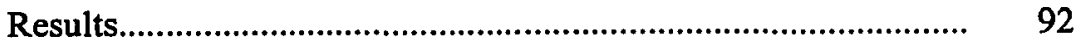

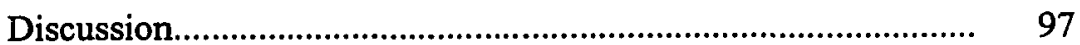

Pilot Study 2.A.................................................................................. 99

Methods................................................................................ 99

Procedure.................................................................... 99

Subjects...................................................................... 99

Results..................................................................................... 100

Reaction Time Analysis.............................................. 100

Error Analysis................................................................. 103

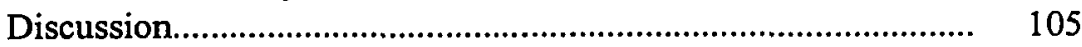

Pilot Study 2.B.................................................................................... 107

Methods............................................................................ 107

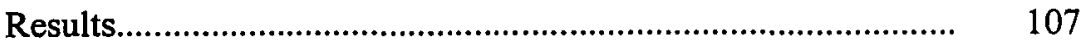

Reaction Time Analysis................................................ 107

Error Analysis.............................................................. 112

Discussion........................................................................... 112

Experiment 2: Broca's Aphasics and Age Matched Controls................... 119

Methods.............................................................................. 119

Procedure ..................................................................... 119

Subjects....................................................................... 119

Results.................................................................................. 122

Reaction Time Analysis................................................ 122

Aphasics Subjects................................................ 126

Old Normal Subjects........................................... 127

Young Normal Subjects...................................... 128

Summary of the RT Results................................. 129

Error Analysis................................................................ 130

Aphasics Subjects.............................................. 131

Old Normal Subjects........................................... 133

Young Normal Subjects..................................... 133

Summary of the Error Results............................ 133

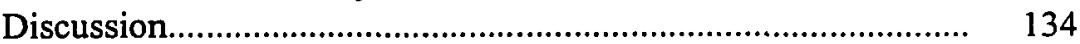

End Notes....................................................................................... 139

General Discussion....................................................................................... 141

The Neely Keefe Three Process Model of Priming ................................. 141

Semantic Priming in Broca's Aphasia................................................... $\quad 150$

Summary and Conclusions...................................................................... 161

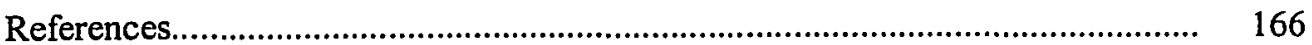

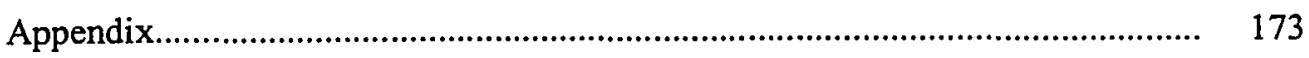

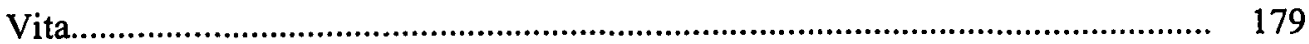




\begin{abstract}
The ability to recognize words is frequently investigated using a lexical decision (LD) priming task in which subjects make rapid yes/no judgements as to whether visually presented letter strings (targets) are words or not. A well established finding is that words are recognized faster when preceded by related words. This "semantic priming effect" is believed to represent both automatic and conscious, strategic driven processes. One factor that influences the degree to which subjects recognize words automatically or consciously, is the time interval between the presentation of the prime and the presentation of the target. This is referred to as the stimulus onset asynchrony (SOA). Automatic processing tends to occur with short SOAs whereas strategic processing is generally observed with longer SOAs. Experiment 1 examined the claims of the Neely and Keefe hybrid three process theory of priming (1989). An attempt was made to dissociate the three priming mechanisms described within this theory (automatic spreading activation, expectancy, and semantic matching) by examining priming across multiple SOAs in a visual pairwise LD task. The results provided clear support for the operation of automatic spreading activation in the short SOA conditions, but less convincing support for strategic processing in the longer SOA conditions.

Experiment 2 examined the nature of automatic versus strategic priming effects in Broca's aphasia. Broca's aphasics, age-matched controls, and young controls participated in a visual pairwise $L D$ task in a 250 SOA condition. A neutral priming condition was included to measure the contribution of facilitatory and inhibitory influences to the overall priming effect. Unlike the young normal controls, the age-
\end{abstract}


matched controls and the Broca's aphasics displayed inhibitory priming effects, indicating the use of strategic processing in this short SOA condition. These results suggest that automatic processing is impaired in Broca's aphasia, and perhaps declines with normal aging as well. Furthermore, they support the hypothesis that subjects will resort to the use of conscious strategies in the LD task, even in short SOA conditions, if unable to process the stimuli automatically.

vii 


\section{INTRODUCTION}

A basic question in psycholinguistic research concerns how humans store and retrieve linguistic information as rapidly as they do. At the level of lexical processing, much effort has been devoted to identifying how much of lexical retrieval is driven by a word stimulus alone, versus how much of lexical retrieval may be facilitated by other contextual information. The lexical decision priming paradigm has been used extensively to investigate the nature of lexical processing in normal subjects and is increasingly being used to examine the nature of lexical processing in aphasic individuals. The typical task in this paradigm involves having subjects make rapid yes/no judgements as to whether target letter strings are words or not. A robust and reliable finding in the literature is the semantic priming effect, first reported by Meyer and Schvaneveldt (1971). This effect refers to the repeatedly demonstrated finding that a lexical decision for a word target is faster when that word is preceded by a related word (the prime) than when it is preceded by an unrelated word. The label 'semantic' priming effect is actually somewhat of a misnomer since the effect has most often been demonstrated with prime-target associates that are syntagmatically related (e. g. doctornurse), as determined by word association norms (Shelton \& Martin, 1992). Nonetheless, positive priming effects have also been demonstrated with prime-target pairs that have a zero association value, but are paradigmatically related (e. g. wifenurse) (e. g. Fischler, 1977).

Semantic priming effects obtained using the lexical decision task ${ }^{1}$ are believed to reflect both automatic and attention driven, strategic processes (den Heyer, Briand, \& 
Dannenbring, 1983; Forster, 1981; Neely, 1977; 1991). These two processes are most frequently discussed within the framework of Posner and Snyder's (1975a, 1975b) twoprocess theory of attention. According to this theory, automatic processing results from automatic spreading activation that is stimulated by the prime. When a prime word is encountered, a node associated with that word is activated, which then automatically spreads to the nodes of related words. Recognition latency is a function of activation level, and therefore, faster responses are observed for related target words as a consequence of their higher levels of activation. Responses to target words unrelated to their prime are unaffected by automatic spreading activation. Automatic priming effects may be considered pre-lexical since word recognition is facilitated by increasing the activation level of word nodes before the target word is actually presented. They may be considered stimulus-driven, since they are observed to occur independent of subject strategies or contextual factors other than the prime-target relationship (e. g. Favreau \& Segalowitz, 1983; Neely 1976, 1977 ).

In contrast, priming effects mediated by strategic processes are relatively slow to enact and may be influenced by a variety of experimental manipulations. According to the Posner and Snyder framework, these effects result when subjects form conscious expectancies about what words may follow primes as targets. Such expectancies have been induced by manipulating the composition of stimuli used in an experiment and by the types of instructions subjects receive. Since strategic effects are influenced by experimental manipulation of contextual factors beyond the prime-target relationship, these are often referred to as contextual effects. Strategic priming has been alternately 
interpreted as either a pre-lexical process that directly impacts word recognition (e.g. Becker, 1980, 1985; Posner \& Snyder, 1975a, 1975b; Neely, 1976, 1977) or as a postlexical process that facilitates the integration of target words once they have been accessed (e.g. Balota \& Lorch, 1986; de Groot, 1984, de Groot et al., 1985; Forster, 1979, 1981; Seidenberg, Waters, Sanders, \& Langer, 1984; Stanovich \& West, 1983).

The lexical decision task is becoming increasingly recognized as a valuable tool for studying lexical processing in individuals with aphasia. Early investigations of lexical processing in aphasia employed explicit, volitional, language tasks, such as those requiring semantic judgements about stimulus triads, or categorization of stimuli (Goodglass \& Baker, 1976; Grober, Perecman, Kellar, \& Brown, 1980; Zurif, Caramazza, Myerson, \& Galvin, 1974). The observation that Broca's aphasics accurately perform these tasks with little difficulty led to the hypothesis that lexical semantic processing was more or less intact in these patients. More recently, the results of studies using the semantic priming lexical decision task have challenged this view. Specifically, investigations using this paradigm have revealed that, unlike non-brain damaged subjects and Wernicke's aphasics, Broca's aphasics display inconsistent evidence of, and even irregular patterns of, semantic priming. In a number of investigations, priming results similar to those obtained with normal subjects have been reported (Blumstein, Milberg, \& Shrier, 1982; Chenery, Ingram \& Murdoch, 1990; Hagoort, 1997; Katz, 1988; Ostrin \& Tyler, 1993; Tyler, Ostrin, Cooke, \& Moss, 1995). However, in some instances, Broca's aphasics have either 1) failed to display a significant priming effect across all conditions tested (Hagoort, 1993; Milberg \& 
Blumstein, 1981; Milberg, Blumstein, \& Dworetsky, 1987), 2) displayed intact, but delayed priming (Prather, Zurif, Stern, \& Rosen, 1992; Prather, Zurif, Love, \& Brownell, 1997), 3) displayed a pattern of reverse priming, in that responses to related targets were slower than responses to unrelated targets (Bushell, 1996), or 4) displayed an unusual pattern of automatic versus strategic effects (Milberg, Katz, Gershberg, \& Brown, 1995). These disparate findings have led to the suggestion that Broca's aphasics may have problems with the automatic access of lexical information (Milberg, et al., 1987). In particular, it has been suggested that automatic lexical access in Broca's aphasics may be "slowed" (Prather, et al., 1992; Swinney, Zurif, \& Nicol, 1989). Other investigators have suggested that it may not be the time course, but rather the strenght of automatic lexical activation that is disturbed in these patients (Milberg, et al., 1995). In contrast to these views which point to a disruption of automatic processing, an opposing view is that attention driven strategic processing involved in postlexical integration is disrupted in these patients (Hagoort, 1997).

Distinguishing between priming effects that are mediated by automatic versus strategic mechanisms may be useful for understanding the nature of lexical impairments in Broca's aphasics. If automatic activation of lexical information is disrupted in these patients, a prelexical locus of impairment involving the decoding and recognition of words may be implicated. Impairment of strategic, conscious attention driven lexical processing could indicate either a prelexical disruption of word recognition itself (depending on one's theoretical stance, e. g. Neely, 1991) due to a reduced ability to use 
contextual information to form expectancies, or a postlexical impairment (e. g. de Groot, 1984; Forster, 1981) in the ability to integrate words once they are accessed.

The primary purpose of the experiments reported here was to examine the nature of automatic versus strategic semantic priming in Broca's aphasics using a visual lexical decision priming paradigm. An additional goal of the proposed research was to examine the claims of a specific theory of semantic priming that attempt to isolate and account for the priming effects mediated by automatic versus strategic processes -Neely and Keefe's (1989) hybrid three process theory.

\section{End Notes}

1. Another task used to study lexical processing is the pronunciation task. Rather than measuring the time it takes subjects to make decisions regarding the lexicality of target words, the time between the presentation of a target and the onset of the subject's pronunciation of the target is measured. Unless otherwise indicated, priming results discussed in this paper will refer specifically to those results obtained with the lexical decision task. 


\section{LITERATURE REVIEW}

\section{Semantic Priming}

The semantic priming effect, as measured with the standard lexical decision task, is typically based on a comparison of reaction times and error rates for recognition of words that follow a related prime versus those that follow an unrelated prime. The typical finding is that responses are faster and more accurate in the related condition compared to the unrelated condition. This "overall" semantic priming effect is often interpreted as evidence that correct responses to word targets are facilitated when they are preceded by a related prime. However, under some conditions, the overall priming effect may actually reflect facilitation of responses to words preceded by a related prime and/or inhibition of responses to words preceded by an unrelated prime (e.g., Becker, 1980; den Heyer, Briand, \& Smith, 1985). To measure the relative contribution of facilitatory and inhibitory effects to the overall semantic priming effect, a neutral priming condition must be used. The purpose of using a neutral prime is to provide a baseline priming condition that is semantically neutral in comparison to the related and unrelated conditions. Most investigators have used a row of XXXXX's or a word such as 'ready' or 'blank' to serve as the neutral prime. Facilitatory effects refer to faster and/or more accurate responses in the related word condition as compared to the neutral priming condition. Inhibitory effects refer to slower and/or less accurate responses in the unrelated word condition as compared to the neutral priming condition.

Posner and Snyder (1975a, 1975b), developed a two-process theory of attention to account for the pattern of facilitatory and inhibitory effects observed across a variety 
of different priming conditions. They noted that facilitation occurred in many situations without inhibition, and that when both processes were observed, facilitatory effects developed earlier than inhibition. Inhibitory effects not only appeared later than facilitatory effects, they were only observed under conditions where subjects appeared to use information about the prime to influence their responses. Posner and Synder argued that early facilitatory effects observed in the absence of inhibition reflected the operation of an automatic attentional mechanism, whereas inhibitory effects signaled the operation of a conscious attentional mechanism that allowed subjects to develop expectations about forthcoming targets based on their conscious awareness of the prime. This two-process model has provided the most popular framework for interpreting automatic versus strategic priming effects. However, as will be discussed below, it now appears that more than one mechanism likely contributes to these inhibitory effects.

\section{Automatic Priming Effects}

Posner and Synder discussed automatic attentional processing in terms of automatic spreading activation (ASA). Priming induced by ASA occurs early on, occurs without a person's intention or awareness, and is not under a person's conscious control. The concept of ASA as a semantic priming mechanism is based on the assumption that semantically/associatively related word nodes are stored or linked closely together in lexical memory. It is presumed that each node has a resting state, and a maximal level of activation that can be triggered if a particular activation threshold is activated. The presentation of a prime activates the threshold for a corresponding node in memory, which then automatically spreads to the nodes of 
related words to activate their thresholds. Once triggered, the maximum level of activation decays rapidly, to return to its resting state. Since this spread of activation only occurs between word nodes that are semantically/associatively related, the presentation of a prime does not impact the processing of words unrelated to the prime. Therefore, ASA should impact the processing of related target words because of their increased activation levels, but should have no effect whatsoever on the processing of unrelated targets. The assumption that priming mediated by ASA is enacted as soon as the prime is presented, and decays rapidly, predicts that the effects of ASA should be observed at short stimulus onset asynchronies (SOAs). This refers to the amount of time between the initiation of the presentation of the prime and the initiation of the presentation of the target. The assumption that priming mediated by ASA occurs independent of subjects' awareness or intentions predicts that ASA mediated priming should be immune to any manipulations that may influence subjects' expectations or use of conscious strategies. Finally, the claim that ASA only influences the activation of related words, but not unrelated words, predicts that there should be no inhibition associated with the recognition of unrelated target words. Therefore, the priming pattern predicted by ASA is facilitation without inhibition. In summary, the manner in which ASA contributes to semantic priming effects predicts that facilitation of related targets without inhibition of unrelated targets should be observed with short SOAs, regardless of any experimental conditions incorporated to encourage subjects' use of conscious strategies. 
Evidence supporting these predictions is well documented in the literature. For example, Favreau and Segalowitz (1983) and Neely (1977) examined facilitatory versus inhibitory priming effects across several different SOAs. In order to distinguish between the priming effects produced purely by ASA from those produced by strategic processes, they specifically manipulated subjects' expectations using a 'category-shift instruction paradigm.' Subjects were told that word targets following the prime bird would be the name of a type of bird "on most trials." However, following the prime body, word targets would usually be the name of something to do with a building. Thus, subjects were instructed to expect stimulus pairs such as bird-robin and bodydoor, but to not expect pairs such as bird-arm and body-heart. These investigators observed that facilitation was determined by semantic relationships alone, regardless of the subjects' expectancies (e. g. for bird-robin and body-heart trials) when prime-target pairs were presented with a $250 \mathrm{~ms}$ SOA. In contradistinction, at this short SOA, neither unexpected nor expected targets that were unrelated their to prime (e. g. birdarm, body-door) produced inhibition relative to a neutral priming condition. This pattern of facilitation without inhibition driven solely by the prime-target relation, regardless of expectations and observed in the short SOA condition was attributed to a fast-acting, inhibitionless automatic spreading activation process.

Additional evidence for distinguishing between automatic versus strategic priming comes from studies in which the relatedness proportion has been manipulated. The relatedness proportion refers to the proportion of related word-prime/word-target trials used in an experiment. A consistent finding in the literature is that the magnitude 
of overall semantic priming effect observed in the lexical decision task increases with increases in the relatedness proportion (de Groot, 1984; den Heyer, 1985; den Heyer, Briand, \& Dannenbring, 1983; Seidenberg et al., 1984; Tweedy \& Lapinski, 1981; Tweedy, Lapinski, \& Schvaneveldt, 1977). A popular interpretation of this relatedness proportion effect is that increases in the relatedness proportion encourage subjects to consciously make predictions, or form expectancies, about what related targets may follow any given prime. This speeds responses to expected, related word targets (facilitation), but slows down responses to unexpected, unrelated word targets (inhibition). The fact that this relatedness proportion effect may be induced by manipulating the composition of stimulus materials used in an experiment, indicates that it is a consequence of strategic rather than automatic processing. Support for the interpretation of ASA as a fast acting, automatic process, that operates free of contextual influences comes from the observation that the relatedness proportion effect is significantly reduced or eliminated with SOAs shorter than $240 \mathrm{~ms}$ (de Groot, 1984; den Heyer, Briand, \& Dannenbring, 1983).

\section{Strategic Priming Effects}

A considerable body of data supports the likelihood that overall semantic priming effects obtained with the lexical decision task often include both a fast-acting, inhibitionless automatic component based on semantic/associative relationships alone, and an inhibition producing, strategic component resulting from subjects' conscious appreciation of the prime (e. g., den Heyer, Briand, \& Dannenbring, 1983). Strategic processes contrast with automatic processes in that they are (1) slow acting, (2) under a 
person's strategic control, (3) cannot occur without a person's awareness and intention, and (4) produce both facilitatory and inhibitory effects. However, the exact nature of strategic priming is not completely understood and it appears that at least two mechanisms, expectancy and postlexical checking, may contribute to strategic priming effects. Several investigators have argued that expectancy operates prelexically because it represents a process that begins to operate before the target word is actually presented. (e. g., Balota, 1983; Becker, 1980; Favreau \& Segalowitz, 1983; Neely, 1977). Others have argued that the pattern of facilitatory and inhibitory effects attributed to expectancy actually reflects influences at a postlexical decision stage (e.g., Forster, 1981; Seidenberg, Waters, Sanders, \& Langer, 1984). In addition, whereas the prelexical versus postlexical nature of expectancy remains unresolved, some priming data clearly require some sort of postlexical checking explanation.

\section{Expectancy}

The basic assumption of expectancy based explanations of strategic priming is that, once the strategy takes hold, subjects use the prime to generate expectations and direct attention to the nodes of likely word targets. If the subsequent target is indeed one of the expected words, reaction times are facilitated. If the target is not an expected word, reaction times are inhibited because subjects must devote more attentional resources to activate the node for a word not present in the expectancy set.

Perhaps the most striking support for a relatively slow acting, inhibition producing expectancy mechanism, that is sensitive to contextual influences, comes from the studies by Favreau and Segalowitz (1983) and Neely (1977) described above. 
Recall that in these studies, subjects' expectancies were manipulated by the types of instructions they received. With $250 \mathrm{~ms}$ SOAs, priming was facilitatory dominant and based on semantic relationships alone, regardless of the subjects' expectancies. However, with SOAs of $700 \mathrm{~ms}$ and greater, a different pattern of results emerged. With longer SOAs, only responses to those targets subjects had been told to expect were facilitated, even if they were unrelated to their prime (e.g. body-door). Furthermore, responses to targets that were unexpected based on the directions that had been given were inhibited, even if they were related to the prime (e.g. body-heart). This combination of facilitatory and inhibitory effects, driven by conscious expectancies rather than prime-target relation, and observed only at long SOAs accords with the basic predictions of conscious, strategic based priming as first elaborated by Posner and Snyder (1975a, 1975b).

Expectancy also provides a plausible explanation for the relatedness proportion effect discussed earlier, by assuming that subjects become more likely to generate an expectancy set for related words as the proportion of related prime-target pairs increases. The result is faster responses to expected (related) targets and slower responses to unrelated targets, which in turn increases the magnitude of overall semantic priming. The facts that 1 ) this effect may be experimentally induced by manipulating the proportion of related-prime target pairs used, and that 2) it is only observed at relatively long SOAs are congruent with the characterization of expectancy as a strategic mechanism. 
Becker $(1980,1985)$ provided a detailed analysis of how expectancy may produce facilitatory and/or inhibitory effects. According to his analysis, when a target is presented, the visual features corresponding to its letters are extracted, which in turn activate a set of word nodes that are visually similar to that target. If a target is preceded by a prime void of semantic context (i. e. a neutral prime), attention is immediately directed to a search of the word nodes in this 'visually defined set,' and word recognition results when a match for the target word is found. However, if the prime preceding the target is a word, an expectancy set, containing nodes of words related in meaning to the prime is generated. When the target appears, attention is first directed to a search of the word nodes activated within this semantically defined expectancy set. If a match for the target is found in the expectancy set, the word is recognized and a response is made. If no match is found after an exhaustive search of the expectancy set, attention is then directed to search the visually defined set. The point to be emphasized in this analysis is that when targets follow word primes, attention is first directed to search through a semantically defined expectancy set. If this search fails (because the target is unrelated, and thereby, not represented in the expectancy set), attention is redirected to search a visually defined set. Following neutral primes however, attention is allocated directly to the visually defined set. This feature of Becker's analysis predicts that lexical decisions to related words will be faster (i.e. facilitated) the smaller the expectancy set is, and that lexical decisions to unrelated words will be longer (i.e. inhibited), the larger the expectancy set is. To examine this hypothesis, Becker (1980) looked at semantic priming with two types of prime-target 
pairs, antonymic word associates (e.g. hot-cold) and category-exemplar pairs (e. g. fruitapple). He reasoned that antonymic associative primes should generate small expectancy sets. Category name primes should generate larger expectancy sets, containing many or possibly all category exemplars. Becker found that for antonymic word associates the priming pattern observed was facilitation without inhibition, as would be expected if recognition of the target depended upon a brief search through a small expectancy set. However, when category-exemplar primes were used (e. g., fruitapple), the priming pattem observed was inhibition with an insignificant amount of facilitation, as would be expected if recognition of the target required a search through a large expectancy set.

These data support the hypothesis that subjects use the prime to generate an expectancy set, and that the relative amount of facilitation and inhibition observed in the overall priming effect may depend upon the predictive value of the prime types used. In a subsequent experiment, Becker (1980) demonstrated that the characteristics of the stimulus list also influence subjects' expectancies. He had obtained the inhibition dominant patterns described above for category exemplar pairs, when they were embedded in a stimulus list consisting of only category exemplar pairs. However, when the prime target pairs included in the stimulus list represented a broader range of semantic relationships (antonymic pairs, common associate pairs selected from association norms, and category-exemplar pairs), category exemplars yielded a facilitation dominant pattern of priming. Specifically, there was a significant $44 \mathrm{~ms}$ facilitation effect for related targets, and a nonsignificant $11 \mathrm{~ms}$ inhibition for unrelated 
targets. Error data indicated that the reaction time data may have underestimated the inhibitory effect due to a speed accuracy trade off. Becker speculated that in the mixed list condition, subjects may have generating fewer expectancies following category primes. This would reduce the overall size of the expectancy set, which would increase facilitation for related targets but decrease inhibition for unrelated targets. In any event, what Becker's results indicate is that both the nature of the prime target relationship, and the characteristics of the stimulus list, influence subjects use of strategic processing.

\section{Postlexical Priming Mechanisms}

The lexical decision data just reviewed supports the characterization of expectancy as a prospective, prelexical mechanism that 1) begins to operate before the target is presented, and 2) directly influences the speed of lexical access. However, another interpretation of these data is possible. Some investigators (e.g. Forster, 1979, 1981; Seidenberg et al., 1984; Stanovich \& West, 1983) have argued that all strategic effects observed with the lexical decision task, including those reviewed above in support of a expectancy based priming, may actually reflect postlexical decision processes that occur after lexical access of the target has occurred. Measurements obtained with the lexical decision task include not only the time required to access a lexical entry, but also the time it takes to make a yes or no decision about the appropriate response, and the time required to execute the response by pressing a response button. Therefore, a plausible interpretation of the data described in support of a prelexical expectancy mechanism is that both expected targets and unexpected targets are processed at the same rate, but that the decision to accept or reject the target as a 
word is influenced by subject expectations. That is, the decision to respond 'yes' to expected word targets may be made with less hesitation than the decision to respond 'yes' to word targets that are unexpected. This would result in the same pattern of facilitation for expected targets and inhibition for unexpected targets as predicted by expectancy. Another possibility is that subjects may assess the semantic/associative relation between the prime and target to assist with their decision. If the prime and target are related, subjects would be biased to decide that the target is a word (since it is impossible for a nonword to be related to its prime) and therefore their correct response to related targets would be facilitated. If the prime and target are not related, subjects would be biased to respond 'no.' Thus correct responses to unrelated word targets would be slowed down (i.e. inhibited) as subjects recover from the incorrect 'no' bias.

Since the results obtained with the lexical decision task are subject to postlexical decision bias arguments such as these, semantic priming has also been examined using a pronunciation task. In the pronunciation task, subjects read aloud target words as quickly as possible, and the critical measure is the time between the target word presentation and the onset of the subject's pronunciation. Since the pronunciation task does not require that subjects make a binary decision about the lexicality of target letter strings, many investigators assume that the results obtained with this task provide a more accurate measure of automatic lexical access (e.g. Balota \& Lorch, 1986; de Groot, 1985; Forster, 1981; Lorch, Balota, \& Stamm, 1986; Keefe \& Neely, 1990; Seidenberg et al., 1984). This view is supported by the observation that inhibitory effects observed with long SOAs for unrelated targets in the lexical decision task are 
either nonexistent or significantly diminished in pronunciation (e.g., Lorch, Balota, \& Stamm, 1986; Keefe \& Neely, 1990).

The pattern of facilitation with little or no inhibition observed in the pronunciation task supports the claim that this task provides a measure of priming that is relatively free of post-lexical effects. Therefore, priming effects that are obtained with the lexical decision task, but not with the pronunciation task, are argued to reflect postlexical decision biases. It is conflicting data obtained with these two tasks that forms the basis for the argument as to whether expectancy operates pre- or postlexically. As noted previously, expectancy has been offered as an explanation for the relatedness proportion effect, an effect that has been demonstrated numerous times with the lexical decision task. Since increases in the relatedness proportion appear to increase priming by encouraging subjects to form expectations about forthcoming targets, many have argued that expectancy operates prelexically. However, Seidenberg, Waters, Sanders, \& Langer (1984) failed to replicate the relatedness proportion effect with the pronunciation task. Based on this finding, they argued that expectancy does not contribute to priming in the pronunciation task, and that the relatedness proportion effect observed in the lexical decision task must originate at the postlexical decision stage.

Recently, this view that expectancy does not operate in the pronunciation task, and the related argument that expectancy must therefore represent a postlexical priming mechanism, have been challenged (e.g. Balota, Black, \& Chenery, 1992; Durgunoglu, 1986; Huttenlocher \& Kubicek, 1983; Keefe \& Neely, 1990; Pring \& Snowling, 1986). 
However, I will defer consideration of this issue for the present and turn to two strategic effects that undoubtedly require a postlexical priming explanation: the backward priming effect, and the nonword facilitation effect. Both of these effects are presumed to result from postlexical processing, because they cannot logically be accounted for by a prelexical process. Interestingly, both of these effects have been observed with the lexical decision task, but not with the pronunciation task.

The backward priming effect (Koriat, 1981; Seidenberg et al., 1984) is observed when asymmetrical associates such as lip stick are used as stimuli. These words are highly related in the forward direction, but not in the backward direction. Backward priming derives from the observation that the second member of such pairs prime the first member. For example, following the prime stick, reaction times are faster to the target lip than to an unrelated word such as sofa. It seems logical to assume that the semantic relation between prime-target pairs such as stick-lip cannot be recognized until after the target has been accessed. Therefore, this effect cannot logically be explained in terms of either automatic spreading activation or a prospective expectancy mechanism. However, the effect is easily explained by a postlexical checking strategy that subjects avail themselves of once both the prime and target have been recognized. Another priming effect that requires a postlexical explanation is the nonword facilitation effect. This is the observation that reaction times to nonword targets are faster when they are preceded by word primes than when they are preceded by neutral primes. This effect cannot be explained by ASA or expectancy, since neither can account for facilitation of unrelated targets. Nonwords are considered unrelated targets 
because in the standard lexical decision paradigm, they are derived by changing one or two letters of word targets unrelated to their primes. Therefore, even in their original form they were unrelated to their primes. Automatic spreading activation cannot explain the nonword facilitation effect, since this process only influences the processing of related targets. Expectancy explanations cannot account for the nonword facilitation effect, since this mechanism would be expected to produce inhibition for unrelated targets, not facilitation. Furthermore, an expectancy mechanism cannot account for the fact that nonword facilitation has been observed for nonwords formed from words from unexpected categories (Neely, 1977; Favreau \& Segalowitz, 1983).

Neely (1991) argues that postlexical processing, which he refers to as "semantic matching," provides a plausible explanation for both the backward priming effect and the nonword facilitation effect. According to his interpretation, semantic matching only applies when targets are preceded by word primes, and may be engaged only after a target's lexical and semantic representations have been activated, but before subjects have had time to complete their yes/no response. Using this strategy, subjects look back to see if the target is related to the word prime that precedes it. If a word target is related to its prime, subjects will be biased to respond yes. This will result in facilitation of 'yes' responses to related target words relative to a neutral priming condition. If a target is unrelated to its prime, subjects will be biased to respond 'no'. For unrelated word targets, this will result in inhibition of correct responses, since subjects must overcome the 'no' bias to provide the correct response that 'yes', the target is a word. For nonword targets, which are always unrelated to their prime, subjects will 
be biased to respond 'no', which in the case of nonwords is the correct response. Thus nonword responses will be facilitated relative to a neutral control condition in which no semantic matching strategy is used. For the most part, the nonword facilitation effect has been observed at relatively long SOAs (den Heyer, Goring, \& Dannenbring, 1983; Favreau, \& Segalowitz, 1983; Neely, 1976; 1977; Neely, Keefe, \& Ross, 1989; but see de Groot, 1984; de Groot, Thomassen, \& Hudson, 1986).

Not only does semantic matching provide a plausible explanation for the nonword facilitation effect and the backward priming effect, it also predicts results similar to those predicted by an expectancy mechanism. That is, both expectancy and semantic matching predict facilitation for related prime-target pairs and inhibition for unrelated prime-target pairs, at long SOAs. Additionally, the semantic matching strategy can account for the relatedness proportion effect and instruction induced priming observed at long SOAs. Semantic matching can account for the relatedness proportion effect if one assumes that as the relatedness proportion increases, subjects become more likely to use information about the relation between primes and targets to bias their lexical decisions. Semantic matching can account for instruction induced priming if one assumes that subjects respond on the basis of similarity between the semantic features of the expectancy generated, and the semantic features activated by the target letter string. For example, when told to expect target words having to do with building parts following the prime body, responses to the target door would be facilitated, since the target matches the semantic features of what subjects have been to led to expect. Similarly, if the target does not meet their expectations (i.e. does not share 
semantic features with their expectation) then they will be biased to respond 'no'. This will inhibit their responses to unexpected word targets (e.g. body-sparrow, body-heart).

That the semantic matching strategy can account for priming effects associated with expectancy based priming, and can also account for priming effects that an expectancy mechanism cannot explain, begs the question as to whether a prospective, pre-lexical expectancy mechanism contributes to strategic priming effects at all. An alternate possibility is that all strategic priming effects are mediated by a retrospective, post-lexical, semantic matching strategy. Indeed, many investigators have aligned themselves with either a prelexical or postlexical interpretation of strategic effects. However, Neely and Keefe (1989) argue that expectancy and semantic matching are distinct mechanisms whose effects can be dissociated under certain experimental conditions. Furthermore, they claim that expectancy, not semantic matching, contributes to semantic priming in the pronunciation task, and therefore must be considered a prelexical priming mechanism. What is of special interest to the experiments to be proposed in this study, is that Neely and Keefe's work suggests a means for dissociating the effects of three different priming mechanisms (ASA, expectancy, and semantic matching) within a single lexical decision experiment. This is an appealing possibility since the use of a pronunciation task is not feasible with aphasic individuals.

The Neely and Keefe Three Process Theory of Priming The automatic spreading activation (ASA) mechanism within the Neely and Keefe (1989) hybrid three process theory is consistent with that described earlier. This 
mechanism mediates automatic priming that is driven by the prime-target relation alone, occurs at short SOAs, and results in facilitation but no inhibition. Expectancy and semantic matching also operate much as has already been described above. Neely and Keefe argue that these are independent, dissociable strategic mechanisms because they may be individually modulated by different contextual influences. They base their argument on evidence obtained in a series of experiments specifically designed to determine the degree to which the relatedness proportion effect is mediated by a prelexical expectancy mechanism, a postlexical semantic matching mechanism, or both. As already noted, both of these strategic mechanisms provide a plausible explanation for the relatedness proportion effect.

Neely, Keefe, and Ross (1989, Experiment 1) sought to replicate the relatedness proportion effect using category names as primes, and high- and low- category dominance exemplars as targets (e.g. bird-robin vs. bird-ostrich). Previously, the effect had been demonstrated using strongly associated prime-target pairs (de Groot, 1984; den Heyer, Briand, \& Dannenbring, 1983; Den Heyer, 1985; Tweedy \& Lapinski, 1981). Neely et al. chose to use high- and low-category dominance exemplar prime targets in order to attempt to dissociate priming effects due to expectancy versus priming due to semantic matching. They reasoned that high-dominance targets should be more likely than low-dominance targets to be included in an expectancy set and that if priming effects are mediated by expectancy, the overall magnitude of priming should be greater for high- versus low-dominance exemplars. Furthermore, if the relatedness proportion effect is mediated by expectancy, increases in the relatedness proportion 
should result in a larger relatedness proportion effect for high-dominance exemplar targets than for low-dominance exemplars targets. In contrast, if the relatedness proportion effect is mediated by a decision biasing semantic matching strategy, increases in the relatedness proportion should impact high- and low-dominance exemplars equally. ${ }^{2}$ Using an SOA of $1000 \mathrm{~ms}$ (which is sufficiently long to expect the contribution of strategic processing), Neely et al. observed significant priming effects for both high- and low-dominance exemplars across three relatedness proportions ( .33 , $.67, .88)$. Collapsed across the three relatedness proportion conditions, reaction time data, but not error data, revealed greater overall priming for high- than for lowdominance exemplars. However, increases in the relatedness proportion resulted in nearly identical increases in priming for both high- and low-dominance exemplars. These results indicate that the standard relatedness proportion effect can be replicated using category names as primes and category exemplars as targets. Moreover, they appear most damaging for an expectancy based explanation for the relatedness proportion effect since increases in the relatedness proportion impacted priming effects for high- and low-dominance exemplar targets equally.

One problem with the interpretation of the results obtained by Neely et al (1989, Experiment 1), is that increases in the relatedness proportion were perfectly confounded with increases in the nonword ratio. The nonword ratio refers to the proportion of trials in which a target is a nonword, given the total number of unrelated word and nonword targets trials. For example, if in a given block of stimuli there are 12 pairs of related words, 6 pairs of unrelated words, and 2 word/nonword pairs, the nonword ratio is $2: 8$ 
or .25 . Neely et al. were the first to point out that these two variables have been invariably confounded in the literature because incremental increases in the relatedness proportion have been consistently accompanied by incremental increases in the nonword ratio. This leads to the possibility that the standard relatedness proportion effect ${ }^{3}$ may actually be due to increases in the nonword ratio, and not due to increases in the relatedness proportion. Since increases in the nonword ratio result in decreases in the number of unrelated prime-target pairs, as the nonword ratio increases, information that the target is unrelated to its prime beccmes an increasingly better predictor that the target is a nonword. The fact that increases in the nonword ratio provide subjects with increased information regarding the semantic relatedness of prime-target pairs increases the likelihood that subjects would use a retrospective semantic matching strategy to bias their lexical decisions. Another observation in Neely et al.'s data that could be attributed to increases in the nonword ratio, and hence to the operation of a semantic matching strategy, is that nonword facilitation increased with increases in the confounded relatedness proportion/nonword ratio. Though these increases were not statistically significant in their data, other investigators had reported statistically significant increases in the nonword facilitation effect with increases in the (confounded) relatedness proportion (de Groot, 1984; den Heyer, Briand, \& Dannenbring, 1983; den Heyer, 1985).

Though the evidence reviewed thus far seems to favor a postlexical interpretation of the standard relatedness proportion effect, Neely et al. (1989) argued that this conclusion was premature since the relatedness proportion and the nonword 
ratio had been repeatedly confounded. They argued that both an expectancy mechanism (modulated by the relatedness proportion), and a semantic matching mechanism (modulated by the nonword ratio) could be responsible for the standard relatedness proportion effect. To tease apart these two variables, they conducted a second experiment (Experiment 2) in which they independently varied the relatedness proportion from .20 to .89 , and the nonword ratio from .08 to .91 across 12 subject groups. They predicted that if use of the semantic matching strategy was indeed modulated by the nonword ratio, increases in the nonword ratio (unconfounded by the relatedness proportion) would lead to increases in priming effects for both high- and low-dominance exemplars and to increases in the nonword facilitation effect. If expectancy based priming was modulated by the relatedness proportion, increases in the relatedness proportion (unconfounded by the effects of the nonword ratio) would have no effect on nonword facilitation, but would produce greater increases in priming for high-dominance exemplars than for low-dominance exemplars. Their results closely supported these predictions. Using a multiple linear regression analysis, Neely et al. observed that priming for low-dominance exemplars and increases in the nonword facilitation effect were significantly affected only by changes in the nonword ratio. Specifically, overall priming for low-dominance exemplars increased by $3.8 \mathrm{~ms}$ for every .10 change in the nonword ratio and nonword facilitation increased by $1.2 \mathrm{~ms}$ for every .10 increase in the nonword ratio. Neither of these effects increased with independent increases in the relatedness proportion. On the other hand, overall priming for high-dominance exemplars was significantly affected by changes in both the 
nonword ratio and the relatedness proportion, with the latter variable producing slightly larger effects. Specifically, overall priming for high-dominance exemplars increased by $2.7 \mathrm{~ms}$ for every .10 change in the nonword ratio, and by $4.1 \mathrm{~ms}$ for every .10 change in the relatedness proportion. Based on these data, Neely, et al. concluded that two separate mechanisms must be postulated to account for the standard relatedness proportion effect: 1) a prelexical expectancy mechanism that contributes to the standard relatedness proportion effect for high-dominance, but not low-dominance exemplars, and 2) a retrospective, postlexical semantic matching mechanism that contributes to the standard relatedness proportion effect for both high- and low-dominance exemplars. Furthermore, whereas expectancy is modulated by increases in the relatedness proportion, semantic matching is modulated by increases in the nonword ratio.

Neely, Keefe, and Ross's (1989) data provide compelling evidence that two dissociable processes (expectancy and semantic matching), independently modulated by the relatedness proportion and the nonword ratio, contribute to the standard relatedness proportion effect. However, they do not provide conclusive evidence that expectancy operates prelexically. In order to test the hypothesis that a prelexical expectancy mechanism contributes to the increased priming for high-dominance exemplars observed by Neely et al. (1989), Keefe and Neely (1990) replicated Neely et al.'s Experiment 1 using the pronunciation task. As noted earlier, many investigators assume that the results obtained with this task are less likely to reflect postlexical decision biases (e.g. Balota \& Lorch, 1986; de Groot, 1985; Forster, 1981; Lorch, Balota, \& Stamm, 1986; Seidenberg et al., 1984). Keefe and Neely reasoned that if postlexical 
priming mechanisms do not operate in the pronunciation task, then only those standard relatedness proportion effects mediated by a prelexical expectancy mechanism should be evident when this task is used, even if increases in the relatedness proportion are confounded with increases in the nonword ratio (as they were in Neely et al., 1989, Experiment 1). Specifically, Keefe and Neely predicted that the priming results obtained with the pronunciation task would reflect increased priming for highdominance exemplars, but not low-dominance exemplars. Priming for low-dominance exemplars should not be observed, since this was modulated by the postlexical semantic matching strategy which would be of no value in a pronunciation task. Furthermore, using the regression analysis data reported by Neely et al., Keefe and Neely were able to predict the exact magnitude of overall priming for high- and low- dominance exemplars. Specifically, they predicted that an increase in the relatedness proportion from .33 to .88 should result in a $22 \mathrm{~ms}$ increase in priming for high-dominance exemplars, but a $0 \mathrm{~ms}$ increase in priming for low-dominance exemplars. Their results closely conformed to these predictions. An increase in the relatedness proportion from .33 to .88 resulted in an 18 to $19 \mathrm{~ms}$ greater overall priming effect for high-dominance exemplars, whereas overall priming was only 1 to 2 ms greater for low-dominance targets. Thus, only those priming effects that Neely et al. had observed with independent manipulations of the relatedness proportion in a lexical decision task, were observed with the pronunciation task. Given the assumption that priming effects measured with pronunciation are free of postlexical influences, Keefe and Neely interpreted these results as evidence that 
increases in the relatedness proportion modulate the use of a prelexical expectancy priming mechanism, not a postlexical priming mechanism. ${ }^{4}$

Independent evidence that expectancy contributes to priming in the pronunciation task comes from studies by Balota, Black, \& Chenery (1992), Durgunoglu (1986, Experiment 5), Pring \& Snowling (1986). These investigators all reported category induced priming with the pronunciation task using the category shift instruction paradigm described earlier. Since postlexical decision processes do not influence priming in pronunciation, these results cannot be explained by a retrospective semantic matching strategy but they are congruent with a prelexical expectancy explanation. Consistent with Keefe and Neely's (1990) data, Durgunoglu (1986) observed instruction induced expectancy based priming for high-dominance, but not low-category dominance exemplars.

To summarize the Neely and Keefe three process theory of priming, Neely and colleagues $(1989 ; 1990 ; 1991)$ argue that in addition to ASA, two dissociable strategic processing mechanisms contribute to semantic priming. ASA mediated priming occurs with short SOAs and results in facilitation only, regardless of any contextual manipulations designed to invoke the use of subject strategies. Strategic priming occurs with long SOAs, results in both facilitation and inhibition, and may be induced by various contextual manipulations. In particular, Neely and Keefe argue that two strategic mechanisms contribute to what has been reported as the standard relatedness proportion effect, expectancy and semantic matching. Expectancy is argued to represent a prelexical strategy that, 1) results in subjects forming expectancies about 
word targets before they are actually presented, and 2) influences the recognition of target words once they are presented. Responses to word targets that are contained in the expectancy set are facilitated, relative to a neutral priming condition. Responses to word targets not contained in the expectancy set are inhibited, relative to a neutral priming condition. It is presumed that the expectancy mechanism is modulated by increases in the relatedness proportion because such increases make it more likely that subjects will use the prime to generate an expectancy set of potential targets. Because it is assumed that high-dominance targets are more likely than low-dominance targets to be included in an expectancy set, expectancy can account for a greater overall magnitude of priming for high- versus low-dominance exemplars, and for greater increases in priming for high-versus low-dominance exemplars as the relatedness proportion increases. Empirical evidence that expectancy operates prelexically comes from those studies that have demonstrated a relatedness proportion effect (Keefe and Neely, 1990) or instruction induced expectancy based priming (Balota, Black, \& Chenery, 1992; Durgunoglu, 1986; Pring \& Snowling, 1985) using the pronunciation task.

The semantic matching strategy represents a post-access checking procedure whereby subjects "look back" to see if a target is related to the word prime that preceded it. Semantic matching is considered a retrospective, postlexical mechanism since it influences priming by biasing subjects yes/no lexical decisions, not by influencing access to the target. If a word target is related to its prime, subjects are biased to respond 'yes,' which results in facilitation of responses to related target words. If a 
target is unrelated to its prime, subjects will be biased to respond 'no'. This results in inhibition of responses to unrelated words and facilitation of responses to (unrelated) nonwords. Therefore, the semantic matching strategy can account for the nonword facilitation effect and overall semantic priming effects. Semantic matching can also account for the backward priming effect. It is assumed that the semantic matching mechanism is modulated by increases in the nonword ratio because such increases provide the subject with more information about prime-target relatedness. Since semantic matching is assumed to impact priming for high- and low-dominance exemplars equally (see Footnote 3 ), semantic matching can account for why increases in the nonword ratio increases priming for both high- and low-dominance exemplars. Furthermore, since the semantic matching mechanism provides an explanation for the nonword facilitation effect, this mechanism can also account for why increases in the nonword ratio result in increases in the nonword facilitation effect.

The purpose of the first experiment to described below, was to determine if these three semantic priming mechanisms may be dissociated in a single lexical decision experiment by examining priming effects elicited with various SOAs. However, before considering the details of Experiment 1, let us turn to a review of semantic priming studies that have been conducted with Broca's aphasics.

\section{Semantic Priming in Broca's Aphasia}

Milberg, Blumstein, and their colleagues (Milberg \& Blumstein, 1981; Blumstein, et al, 1982; Milberg, Blumstein, and Dworetzky, 1987) were the first to observe abnormal semantic priming patterns in Broca's aphasics. When semantic 
priming was measured using the standard visual pairwise lexical decision task ${ }^{5}$, Broca's subjects failed to display a statistically significant semantic priming effect (Milberg \& Blumstein, 1981), however, they did show evidence of significant priming when an auditory pairwise lexical decision task was used (Blumstein, et al., 1982). Furthermore, although Wernicke's aphasics and normal age matched controls displayed overall semantic priming with the auditory triplet lexical decision paradigm, Broca's subjects did not (Milberg, Blumstein, and Dworetzky, 1987). Milberg and his colleagues suggested that these anomalous results could represent either an impairment at the level of lexical representation, or a deficit to the processes involved in accessing lexical information. They favored the latter hypothesis since an impaired lexical representation hypothesis could not account for the normal priming that was demonstrated by Broca's aphasics under some experimental conditions (e. g., Blumstein et. al, 1982; Katz, 1988). Nor could an impaired lexical representation hypothesis explain why Broca's patients tend to perform well on semantic judgement tasks (e.g., Goodglass \& Baker, 1976, Milberg et. al, 1981). Since Broca's aphasics displayed intact lexical knowledge under some conditions, but did not consistently use this information to rapidly access information under priming conditions, Milberg and colleagues suggested that the nature of their impairment likely involved difficulty in accessing lexical semantic information via "automatic" processing routines. Since that time, much of the debate regarding how to interpret the priming results obtained with Broca's aphasics has centered around the automatic versus strategic processing distinction. 
Though it is frequently reported that the priming performance of individuals with Broca's aphasia is inconsistent, if one carefully examines the task materials and experimental paradigms used across studies, it becomes clear that this claim only applies to a subset of the investigations reported to date. Table 1 provides a summary of the semantic priming results obtained with Broca's aphasics and indicates the experimental paradigm and interstimulus interval (ISI) or stimulus onset asynchrony (SOA) that were used (see note 6) As can be seen in Table 1, all investigations using an auditory, pair-wise, lexical decision task have obtained significant overall priming results from Broca's aphasics (Blumstein, et al., 1982; Chenery, et al., 1990; Katz, 1988; Milberg, et al., 1988; Milberg et al. 1995; Ostrin \& Tyler, 1993; Tyler, et al., 1995). Investigations using the auditory, triplet, lexical decision task have yielded less consistent results. Using this paradigm and an ISI of $500 \mathrm{~ms}$, Milberg, et al. (1987) failed to find semantic priming in Broca's subjects. However, Hagoort (1993) reported intact priming at 100 and $500 \mathrm{~ms}$, but not at $1250 \mathrm{~ms}$ with the auditory triplet paradigm. Overall priming effects obtained with the visual, pair-wise, lexical decision task have also yielded inconsistent results. Bushell (1996), and Milberg and Blumstein (1981) failed to find normal priming performance in Broca's subjects using this paradigm with SOAs of $1500 \mathrm{~ms},>2000 \mathrm{~ms}^{6}$, and $2500 \mathrm{~ms}$, but Hagoort (1997) reported normal priming with SOAs of $300 \mathrm{~ms}$ and $1400 \mathrm{~ms}$. In contrast, intact, but delayed priming has been reported in two Broca's aphasics when priming was examined with a visual, listpriming, lexical decision task (Prather, et al.,1997; Prather, et al., 1992). With this latter 
Table 1. Summary of semantic priming results obtained with Broca's aphasics.*

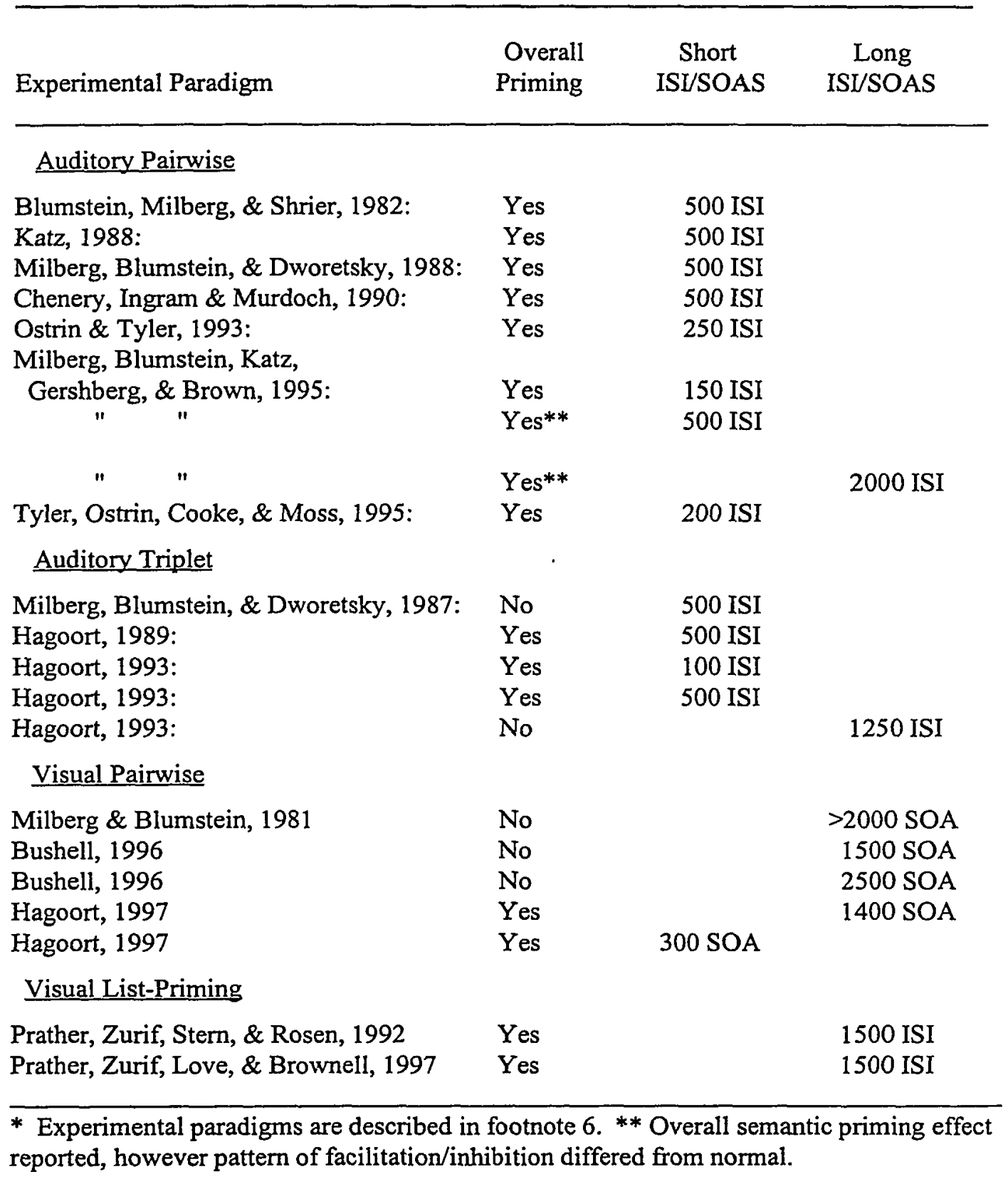


paradigm, Broca's displayed overall semantic priming with an ISI of $1500 \mathrm{~ms}$, whereas elderly and college aged controls displayed overall semantic priming with ISIs ranging from 500 to $800 \mathrm{~ms}$.

Of course, the investigations cited here vary in more details than simply the type of priming paradigm and the ISI/SOA that were used. Nonetheless, this analysis allows for a few preliminary observations. First, Broca's patients prime more consistently when an auditory stimulus presentation is used, especially when stimuli are presented in pairs rather than triplets. Second, they prime less consistently when a visual pairwise lexical decision task is used than when a visual list-priming task is used. Third, if we carefully examine the results obtained with the pairwise and triplet paradigms, we see that with one exception (Milberg et al., 1987), a significant overall priming effect was reported when an ISI or SOA of $500 \mathrm{~ms}$ or less was used. When an ISI or SOA of 1400 ms or greater was used, significant priming was reported in only two (Hagoort, 1997; Milberg et. al, 1995) of six experiments. Fourth, in those cases where priming has been examined in Broca's aphasics using a paradigm that purportedly reduces strategic effect, (i.e. the visual list priming paradigm), significant priming was observed, but at a longer than normal prime-target interval.

To summarize these data further, we can see from Table 1 that in fact, overall semantic priming has been reported to be absent in Broca's aphasics only under the following conditions: 1) when targets were preceded by two primes (i.e. with the auditory triplet paradigm) in combination with an ISI/SOA of $500 \mathrm{~ms}$ or greater (Hagoort, 1993; Milberg et al., 1987), and 2) when the SOA between primes and targets 
in a pairwise visual presentation was $1500 \mathrm{~ms}$ or greater (Milberg \& Blumstein, 1981, Bushell, 1996). Furthermore, an overall priming effect has been reported with an SOA as short as $300 \mathrm{~ms}$ with the visual pairwise paradigm, and with an ISI as short as 150 ms with the auditory pairwise paradigm (Hagoort, 1997; Milberg, et al., 1995, see Table 1). If automatic, but not strategic, processing is measured at short ISI/SOAs, these data suggest that the locus of Broca's aphasics' lexical processing impairment has more to do with stategic, rather than automatic processing. This is the argument made by several investigators who claim that current evidence fails to support an automatic access deficit (Hagoort, 1997; Ostrin \& Tyler, 1993; Tyler et al., 1995).

Other investigators have suggested that automatic processing is intact, but slowed down in Broca's aphasia. Swinney, Zurif, and Nicol (1987) examined priming in Broca's aphasics using a cross-modal lexical decision paradigm. With this task, subjects made lexical decisions to visual letter strings while simultaneously listening to auditorily presented sentence contexts. The lexical decision targets were related to one of two meanings of ambiguous words contained in the sentences. Normal subjects demonstrated priming for words related to both meanings of the ambiguous words. In contrast, the Broca's aphasics only demonstrated priming for the most frequent meaning of these words, regardless of the prior sentence context. Swinney et al., argued that automatic lexical access in Broca's aphasics operates on a "slower-than-normal rise time, only the most frequent meaning representation being engaged within the time frame imposed by the experimental paradigm" (p. 32). Prather and colleagues (1992, 1997) argue that their data also support the hypothesis of intact, but slowed automatic 
activation in Broca's aphasia. Recall that they examined priming in Broca's aphasics using a visual, list priming task and observed that Broca's aphasics displayed priming with an ISI of $1500 \mathrm{~ms}$. This was in contrast to young and age-matched controls who displayed priming with ISIs ranging from 500 to $800 \mathrm{~ms}$.

Prather and colleagues $(1992,1997)$ argue that priming data obtained with the pairwise and triplet lexical decision paradigms, even with short SOA/ISI conditions, are inadequate for making claims about the integrity of automatic processing, unless an internal check is used to specifically rule out the contribution of strategic processing. One way of providing such an internal check would be to examine the relative contributions of facilitatory and inhibitory effects to the overall priming effect. This of course would require the use of a neutral priming condition against which to measure the effects obtained in related and unrelated word pair conditions.

To date, only one lexical priming study involving Broca's aphasics has used a neutral context to examine the combined influence of facilitatory and inhibitory effects on overall semantic priming. Milberg, et al., (1995) examined the extent to which the auditory pairwise lexical decision performance of aphasic patients could be influenced by contextual manipulations known to induce strategic processing in normals. In one experiment, they manipulated prime-target predictability ${ }^{7}$ while holding the ISI constant at $500 \mathrm{~ms}$. In the second experiment, prime-target predictability was held constant and prime target pairs were presented with a short ISI $(150 \mathrm{~ms})$ and a long ISI (2000 ms). The relatedness proportion was .50 in both experiments. To distinguish between automatic effects and strategic effects, they used a neutral priming condition to observe 
the relative contributions of inhibition and facilitation to the overall priming effect. Neutral primes were nonwords such as "yandle" and "pircle." Strategic processing, as indicated by a priming pattern of facilitation and inhibition, would be expected in the high prime-target predictability and long ISI conditions, whereas automatic processing, as indicated by a priming pattern of facilitation without inhibition, would be expected in the low probability and short ISI conditions.

Broca's aphasics displayed significant overall priming in all conditions, as did normal subjects. Furthermore, similar to young normal controls, Broca's patients displayed 1) facilitation and inhibition in the first half of the high predictability condition, 2) facilitation without inhibition in the second half of the high predictability condition, and 3) facilitation without inhibition in the short ISI condition. Surprisingly however, unlike young and old controls, they displayed inhibition without facilitation in the first half of the low predictability condition, and facilitation without inhibition in the long ISI condition of Experiment 2. Therefore, even though Broca's aphasics displayed significant overall priming across all conditions, in two conditions the pattern of combined facilitatory and inhibitory effects that contributed to the overall priming effect was different than that observed in normals. Specifically, Broca's aphasics 1) failed to display inhibition in a long SOA condition, where strategic effects were expected, 2) failed to show facilitation in a low predictability condition wherein facilitation due to automatic activation was expected, and 3) displayed inhibition in a low probability condition where strategic effects were not expected. 
The results observed in the low probability condition were particularly informative. In the experimental trials immediately following the low probability induction set, control subjects displayed a facilitation effect, but control subjects did not. However, by the second half of the experiment, all three groups displayed a signficant facilitation effect. Milberg et al. argued that the facilitatory effect produced by Broca's subjects in the second half of the experiment must have resulted from their developing a strategy, not ASA. If the effect were due to ASA, facilitation should also have been observed in the first half of the experiment (as it was in the normal controls), since ASA occurs independent of contextual manipulations. Based on these results, Milberg et al. hypothesized that the activation levels of lexical nodes are reduced in Broca's aphasics. A reduction in the activation of word nodes within a semantic network would result in less consistent, and decreased magnitudes of facilitation, especially in a task not conducive to the use of strategic processing. Thus, Milberg et al. argued that facilitation was not observed immediately following the low induction set because the lexical entries for target words were not sufficiently activated, and because there was inadequate information available for them to develop a strategy. However, after being exposed to semantically related pairs during the first half of the experiment, they were able to use this information to develop a strategy that resulted in facilitation in the second half of the experiment. This intrepretation seems reasonable. However, it becomes more complex when one considers the explanation offered for why Broca's aphasics (unlike normal controls) displayed an inhibitory effect immediately following the low probability induction set: 
"However, the appearance of inhibition in the first half of the low induction set is particularly important.... What these findings suggest is that the Broca's aphasics rely on heuristics even in the absence of "information" from the data set to help them determine whether the particular heuristic is appropriate." (p. 43)

Thus, they argued that facilitation was not observed immediately after the low induction set due to both reduced lexical activation levels and the inability to develop a strategy. However, the anomalous inhibition effect observed in the same condition was attributed to the ability to develop a strategy in the presence of limited contextual cues. This argument seems implausible.

Milberg et al., suggested that Broca's aphasics, in comparison to normal controls, display an "overreliance" on the use of conscious strategies. Furthermore, they suggested that this overreliance on strategic processing may have previously masked their impairment in automatic processing. For example, they attributed the facilitation without inhibition produced by the Broca's aphasics in the 150 ISI condition of experiment 2 to their ability to use strategic processing even at that short ISI. This implies that previous reports of intact priming effects in Broca's aphasics were actually due to their ability to use strategies to perform the task, rather than intact automatic activation. However, one inconsistency in Milberg et al.'s arguments is that they provided no clear account as to why an inhibition effect was not observed in either the 150 or the 2000 ISI conditions, even though they clearly stated that they attributed priming in both of these conditions to controlled processing. Thus, they appealed to the overuse of strategies to explain the inhibition observed in the low probability condition, but failed to account for the absence of inhibition in the 150 and 2000 ISI conditions, 
other than to suggest that Broca's aphasics may use an "evolving set of heuristics, some of which are less likely to produce inhibition than others" (p. 43).

Clearly the data reported by Milberg et al. (1995) suggest that Broca's aphasics do not display the same sensitivity to facilitatory and inhibitory contextual influences as do normal subjects, even when overall significant priming effects are observed. Additional evidence that Broca's aphasics do not show the same strategic processing as normal control subjects comes from data reported by Bushell (1996). Bushell looked at semantic priming across two relatedness conditions to determine if the standard relatedness proportion (RP) effect would be observed with Broca's subjects. Stimulus onset asynchronies were sufficiently long to ensure that strategic processing was likely ( $\geq 1500 \mathrm{~ms})$. In a low RP condition (.20), Broca's subjects, like normals, did not show priming. As expected, normal subjects displayed an increase in semantic priming in a high RP condition (.80). However, Broca's subjects displayed reverse priming in the high $R P$ condition. That is, their responses to related word pairs was slower than their responses to unrelated word pairs. Bushell concluded that controlled processing in her Broca's subjects was abnormal, possibly resulting from a struggle to access the prime's representation in anticipation of generating an expectancy for related targets.

The fact that Milberg, et al. (1995) observed significant overall priming, but abnormal patterns of facilitation and inhibition with both short and long SOAs, raises the question as to whether abnormal facilitatory and inhibitory influences contributed to the supposedly normal overall priming results that have been reported in previous studies that did not specifically look at these two factors (Blumstein, et al, 1982; 
Chenery, et al., 1990; Hagoort, 1997; Katz, 1988; Ostrin \& Tyler, 1993; Tyler, et al., 1995). Clearly the data reported by Milberg et al., (1995) and Bushell (1996) suggest that strategic processing differs from normal in Broca's aphasia. What is less clear is whether or not automatic priming is intact in Broca's aphasia, as has been claimed by those investigators who have obtained significant overall priming effects when short SOA/ISIs were used (Hagoort, 1997; Ostrin \& Tyler, 1993; Tyler, et al., (1995).

Another manner in which to observe the relative contributions of automatic versus strategic effects to overall semantic priming would be to examine the relative pattern of facilitatory and inhibitory effects across both short and long SOA conditions. Prather and Swinney (1988) have claimed that there is a brief temporal window during which ASA mediated priming operates immune from contextual influences, although the beginning and end points of this temporal window vary with the research paradigm used. The purpose of the series of experiments presented in the current paper was to determine whether the temporal window for automatic versus strategic priming effects is the same in Broca's aphasics as it is in normal subjects. Experiment 1 examined the time course of automatic versus strategic priming effects across multiple SOAs using normal subjects, to serve as a baseline for Experiment 2. Experiment 2 examined whether the same pattern of facilitatory and inhibitory effects observed in normal subjects could be obtained with Broca's aphasics.

\section{End Notes}

1. Neely (1991) provides a comprehensive review of eighteen "core phenomena" reported in the semantic priming literature which require explanation. Within that review, Neely argues that the Neely-Keefe hybrid three process theory can account for 
all but three of these effects. The present discussion will only review those patterns of priming data pertinent to the proposed experiments.

2. This assumes that before subjects have completed their yes/no response, they always have time to access and recognize the relation between low-dominance exemplars and their category. The point to be emphasized is that unlike with an expectancy mechanism, there is no reason to assume that the semantic matching strategy would differentially affect the priming of high-versus low-dominance exemplars, provided there is sufficient time for the mechanism to operate after target recognition is complete, and before a response has been given.

3. The expression, "standard relatedness proportion effect" will be used to refer to increases in overall semantic priming that has been observed in those studies wherein the relatedness proportion and nonword ratio have been confounded. As will become evident, Neely and colleagues argue that this effect is actually modulated by both increases in the relatedness proportion and the nonword ratio. The former modulates expectancy and impacts priming for high-dominance exemplars only. The latter modulates semantic matching and impacts priming for high- and low- dominance exemplars.

4. Notice that Keefe and Neely's observation of a relatedness proportion effect for highbut not low-category dominance exemplars with the pronunciation task is at odds with Seidenberg, Waters, Sanders \& Langer's (1984) failure to find a relatedness proportion effect with the pronunciation task. Keefe and Neely suggested that the relatedness proportion manipulation that Seidenberg et. al., used (from .20 to .33) may have been too small to elicit the standard relatedness proportion effect.

5. Four different lexical decision paradigms have been used to study semantic priming in Broca's aphasics. These are summarized in Table 1. The visual pairwise lexical decision paradigm refers to the standard lexical decision task wherein subjects make decisions about visually presented letter string targets preceded by a single prime. The auditory pairwise lexical decision task is similar, except primes and targets are presented auditorily. In the auditory triplet lexical decision task, subjects make lexical decisions about a single target word or nonword that is preceded by two consecutively presented primes. This task is used to determine sensitivity to multiple meanings of ambiguous words. The SOAs (stimulus onset asynchronies) reported for the visual pairwise paradigms refer to the time interval between the onset of the prime and the onset of the target. Thus the SOA includes both the prime duration and the interstimulus interval (ISI) which is the interval of time between the offset of the prime and the onset of the target. For auditory lexical decision tasks, typically only the ISI is reported, since the prime duration for auditorily presented stimuli cannot be controlled as a constant time interval. In the list-priming lexical decision task, visual letter strings are presented in a continuous list format. The ISI is varied between each stimulus and 
subjects respond to all stimuli. The logic behind this task is that subjects will be less likely to look for a relationship between words that are not obviously grouped together as pairs or triplets, and therefore, will be less likely to use strategic processing to complete the task. Some investigators have argued that this task provides a more accurate measure of automatic processing than do the pairwise or triplet presentations (e.g., Shelton \& Martin, 1992). For the two list-priming studies reported in Table 1, each letter string (i.e. primes and targets) remained on the screen until a response was made, or for a maximum of $2000 \mathrm{~ms}$.

6. In the Milberg and Blumstein (1981) study, subjects made lexical decisions to both primes and targets stimuli. These authors report that there was "approximately a 2-sec interval between prime and target word presentations and a $4 \mathrm{sec}$ interval between trials" (p. 376). Exposure durations for the primes and targets were not reported. It may be inferred that the total SOA exceeded $2000 \mathrm{~ms}$ if the interval between prime and target stimuli was close to 2 seconds, since the total SOA would also have to include the duration of the prime and the subject's reaction time to respond to the prime.

7. Milberg et al., (1987) used a novel approach to manipulate prime-target predictability in Experiment 1. Prior to the onset of experimental trials in the high probability condition, subjects were presented with a series of trials in which all real word pairs were semantically related. This was referred to as the high probability induction set. For the low probability condition, experimental trials were preceded by a low probability induction set. In this set, none of the word pairs were semantically related. To examine how these induction sets affected priming over the course of the experiment, data obtained in the first half of the experimental trials was analyzed separately from those obtained the second half. 


\section{EXPERIMENT 1}

The objective of Experiment 1 was to examine whether the effects associated with each of the three priming mechanisms described within the Neely and Keefe hybrid three process theory could be dissociated when priming effects were measured across multiple SOAs of increasing duration. One aspect of Neely and Keefe's hybrid three process theory critical to the proposed experiment is the fact that the operation of the three processes is temporally ordered. Since automatic spreading activation is enacted more rapidly than strategic priming mechanisms, effects associated with automatic spreading activation should appear earlier (i.e. at shorter SOAs) than effects associated with strategic mechanisms. Furthermore, Neely and Keefe's interpretation of the expectancy mechanism as a prelexical process implies that this strategy operates sooner than the semantic matching strategy, since the later is available only after word recognition has occurred.

Experiment 1 examined the overall contribution of facilitatory and inhibitory effects to overall priming across nine separate SOA conditions, ranging from $150 \mathrm{~ms}$ to $950 \mathrm{~ms}$. Both a high relatedness proportion and a high nonword ratio were used to induce strategic processing. In order to dissociate the effects of expectancy based priming and strategic matching, category-name primes and high- and low-dominance targets were used. Based on the Neely and Keefe model, three predictions were made. First, facilitation without inhibition was expected in the shortest SOA conditions. This would be consistent with the operation of a fast acting automatic spreading activation mechanism that operates without strategic influences, and contributes to overall priming 
effects by facilitating the processing of related targets without influencing the processing of unrelated targets. Second, inhibitory effects were expected to be observed in all but the shortest SOA conditions, as an indication that subjects were engaged in strategic processing. Third, provided the time span in which these two mechanism operate was sufficiently distinct to be distinguished across the SOAs examined in this experiment, significant inhibitory effects for high-dominance exemplars were expected to appear before (i.e. with shorter SOAs) significant inhibitory effects for lowdominance exemplars and before significant nonword facilitation effects were observed.

\section{Methods}

\section{Stimulus Materials and List Construction}

A base list of 360 prime-target trials containing 180 nonrepeated word targets and 180 nonrepeated nonword targets was constructed as follows. Eighteen categories that could be represented as a single word (e.g. clothing) were selected from the Battig and Montague (1969) norms of free associations to categories. These 18 category names served as word primes, and were used an equal number of times preceding a word or a nonword target. Ten category exemplars, were selected from each of these categories to serve as word targets. Five category exemplars were chosen from among the six most frequent responses in each of the 18 category rank lists to serve as highcategory dominance $(\mathrm{HCD})$ exemplars. The mean response frequency for the total number of HCD exemplar targets was 288.29. These were matched with five low category dominance (LCD) exemplars chosen from each of the 18 category rank lists that matched the HCD exemplars in terms of word length. The mean response 
frequency for the total number of LCD exemplar targets was 21.31. Target word length ranged from 3 to 9 letters with an average target word length of 4.92 . There were insufficient numbers of high- and low- category dominance exemplars within each category to match the high-and low dominance targets for word frequency. The mean frequency of occurrence counts (Kucera and Francis, 1967) for the complete set of HCD and LCD exemplar targets were 71.09 and 13.08 , respectively.

Orthographically and phonologically legal nonword targets were created by substituting and/or transposing one or two letters of category exemplars not selected for word targets. These nonword targets never followed a prime that was the name of the category of which the word used to create the nonword was a member. Nonword targets were matched with word targets for word length.

Of the 180 word targets, 120 were preceded by a category name (word) prime and 60 were preceded by the neutral prime 'blank.' To achieve a relatedness proportion of .80 for the word prime/word target pairs, 96 of the 120 word primes preceded word targets that were exemplars of the category named by the prime (e. g., color-blue). These were designated as related word-target pairs. The remaining 24 word prime/word target pairs were created by matching category exemplars with a category other than the one the exemplar was selected from (e.g., flower-wool). These were designated as unrelated word-target pairs. The remaining 60 word targets were preceded by the neutral prime, 'blank,' and were designated as neutral word-target pairs. Of the 180 nonword targets, 120 were preceded by word primes (category names), and 60 were preceded by the neutral prime 'blank.' These were designated as word-NW pairs and 
neutral-NW pairs, respectively. Each individual category name prime occured an equal number of times preceding a word or nonword target. Half of the word targets in each priming condition were low category dominance exemplars (LCD), and half were high category dominance (HCD) exemplars. Half of the nonword targets were derived from HCD words, and half were derived from LCD words. A list of stimulus items used, including their category association frequencies and word frequencies, are provided in the Appendix.

In summary, the ratio of word-prime/word-target pairs to neutral-prime/wordtarget pairs contained in the base list was 120:60. The ratio of word-NW pairs to neutral-NW pairs was also 120:60. The ratio of related, unrelated, and neutral wordtarget pairs was $96: 24: 60$. These proportions yielded a nonword ratio of .83 , which is the ratio of word-NW target pairs (120) to the total number of word-NW target pairs plus unrelated word-target pairs $(120+24)$ and a relatedness proportion of .80 as described above.

A subset of the total 360 prime-target pairs contained within the base list were designated as critical for purposes of statistical analysis and counterbalancing. There were 24 (12 high- and 12 low-dominance) critical observations for each word-target priming condition (related, unrelated, and neutral) and 24 critical observations for each nonword-target priming condition (word-NW and neutral-NW). This resulted in a total of 120 critical prime-target observations contained within the entire set of 360 primetarget pairs. The remaining prime-target pairs serve as fillers and were included to achieve the desired relatedness proportion and nonword ratio described earlier. 
To counterbalance critical target items across the different priming conditions, five additional list versions were constructed from the base list. These were designated as lists $A, B, C, D, E$, and $F$ for identification purposes. These additional lists were formed by exchanging the items that served as primes for the critical targets. For the critical conditions only, prime type was alternated across the six list versions such that each critical word target appears twice each in the related, unrelated, and the neutral priming conditions and each critical nonword target appears three times in the word and the neutral priming conditions. As noted in Table 2, lists $\mathrm{A}, \mathrm{C}$, and $\mathrm{E}$ contained the same word-NW pairs and neutral-NW pairs, but differed across the three priming conditions matched with word targets. For example, the target cotton was preceded by the related prime cloth, in list $\mathrm{A}$, by the neutral target blank, in list $\mathrm{C}$, and the unrelated target fruit, in list $\mathrm{E}$. Lists $\mathrm{B}, \mathrm{D}$, and $\mathrm{F}$ were identical to lists $\mathrm{A}, \mathrm{C}$, and $\mathrm{E}$, respectively, with regard to prime-word target pairs, but the primes for NW targets were reversed. Therefore, whereas in lists $\mathrm{A}, \mathrm{C}$, and $\mathrm{E}$, the NW target pamb was preceded by a word prime, in lists $\mathrm{B}, \mathrm{D}$, and $\mathrm{F}$, it was preceded by the neutral prime, blank. Primes for filler items were left unchanged across the six list versions. These six versions of the stimulus lists were counterbalanced across subjects within each SOA group.

Each list version was subdivided into 12 blocks of 30 prime-target pairs containing the same ratio of each type of prime target pair as that represented in the complete list. Prior to each experimental session (i.e. for each individual subject), the order of items within each of these blocks was randomized, then the order of the 12 blocks was randomized. Scrambling the stimuli in this manner increased the likelihood 
Table 2. Word and NW prime-target conditions across stimulus list versions. Stimulus lists $\mathrm{A}, \mathrm{C}$, and $\mathrm{E}$ contain the same NW pairs, but across the three lists, word targets are paired with three different priming conditions. Lists $B, D$, and $F$ are identical to lists $A$, $\mathrm{C}$, and $\mathrm{E}$, with regard to prime-word target pairs, but primes for NW targets are reversed.

\section{Word Target Conditions $\quad$ NW Target Conditions}

\begin{tabular}{|c|c|c|c|c|c|c|}
\hline List A & cloth & COTTON & body & PAMB & blank & GOCK \\
\hline List $C$ & blank & COTTON & body & PAMB & blank & GOCK \\
\hline List $E$ & fruit & COTTON & body & PAMB & blank & GOCK \\
\hline List B & cloth & COTTON & blank & PAMB & body & GOCK \\
\hline List D & blank & COTTON & blank & PAMB & body & GOCK \\
\hline List F & fruit & COTTON & blank & PAMB & body & GOCK \\
\hline
\end{tabular}


that the critical conditions were evenly distributed across the experiment. Also, since no two subjects received the same sequence of items, the possibility of extraneous serial effects such as practice or fatigue were minimized.

A practice block of 48 prime-target trials containing 24 word targets and 24 nonword targets was constructed in a manner similar to that used to construct the experimental base list. Primes and targets for the practice block were selected from four categories not used in the experimental list. Half of the target words represented high category dominance exemplars, and half represented low category dominance exemplars. Eight word targets were preceded by related (category name) primes, eight were preceded by unrelated (other-category name) primes, and eight were preceded by the neutral prime 'blank.' Twelve of the nonword targets were preceded by word primes, and twelve were preceded by the neutral prime 'blank.' The implication of using a relateness proportion and nonword ratio in the practice block (.50 and .60 , respectively), that differed from those used in the experimental trials, will be addressed in the general discussion.

\section{Procedure}

All prime target pairs were presented consecutively on the center line of a Dell computer monitor. Stimulus presentation was controlled by a Dell pentium processor via DMASTR software'. Subjects responded by pressing the left and right keys of a mouse wired to interface with the Dell processor via a Metrabyte parallel I/O port. The DMASTR software polls this device every millisecond, thus allowing for the synchronization of stimulus presentation and the measurement of reaction times within 
one millisecond accuracies. Pressing the right key of the mouse generated a 'yes' response, pressing the left key generated a 'no' response. All subjects responded by pressing these keys with the first two fingers of their nondominant hand.

Nine SOA intervals, ranging from $150 \mathrm{~ms}$ to $950 \mathrm{~ms}$ were varied between subjects. Across the nine SOA conditions, the interstimulus interval (ISI) was held constant at $50 \mathrm{~ms}$. This refers to the time interval from the offset of the prime to the onset of the target, during which the computer screen is blank. SOA intervals were varied by increasing the prime duration by increments of $100 \mathrm{~ms}$ for each condition. The prime duration is the time interval the prime is visually present on the screen. Thus in the shortest SOA condition (150 ms), the prime duration was $100 \mathrm{~ms}$ and the ISI was $50 \mathrm{~ms}$. In the $250 \mathrm{SOA}$ condition, prime duration was $200 \mathrm{~ms}$, in the $350 \mathrm{SOA}$ condition the prime duration was $300 \mathrm{~ms}$, and so on. Primes were presented in lowercase letters, targets were presented in uppercase letters. The target remained on the screen for $4000 \mathrm{~ms}$ or until the subject responded, whichever occurred first. The subsequent prime appeared $2000 \mathrm{~ms}$ (intertrial interval) after the previous target was cleared from the screen. If a subject failed to respond to a target within $4000 \mathrm{~ms}$, that item was recorded as a no response error, the intertrial interval was initiated, followed by presentation of the subsequent prime.

Subjects were read general instructions describing the task. These instructions were also presented visually on the computer screen, prior to presentation of 48 practice items. Subjects were told that they would see pairs of letter strings appear on the computer screen, and that they were to decide as quickly and as accurately as possible, 
whether or not the second letter string was a word or not. It was emphasized that they should try to silently read each of the letter strings, but to respond only to the second in each pair, which was in uppercase letters. Subjects were told that the target would automatically clear from the screen if they did not respond within four seconds. They were asked not to respond to one of the practice items so that they could see that they were being given a reasonable amount of time in which to respond. They were encouraged to respond as quickly as possible, without sacrificing accuracy. Practice items were repeated as necessary until the examiner was convinced that the subject understood the task. Test materials were presented in 12 blocks of 30 prime-target pairs. Subjects were given a break not to exceed one minute following each block of test materials.

\section{Subjects}

A total of 162 undergraduate psychology students from the Louisiana State University served as subjects. All subjects were right handed, native and monolingual speakers of English. Subjects demonstrated sufficient visual acuity (aided or unaided) to read stimulus words presented white on black, such as those used in the experiments. This was determined by having subjects match five words printed on paper (black on white) to words presented on the computer screen (white on black). No subjects had a history of neurological disorder, psychiatric disorder, or drug or alcohol abuse. Each subject was assigned to one of the nine SOA groups. Eighteen subjects participated in each experimental group. As noted previously, presentation of the 6 list versions was counterbalanced across the subjects in each group. All subjects received extra credit for 
participation in this study. All subjects whose overall error rate exceeded $10 \%$ were replaced, and their data were discarded

\section{Results}

Recall that for purposes of statistical analysis 24 target items were designated as critical for each priming condition. There were three priming conditions for wordtargets (related, unrelated, and neutral), and two priming conditions for nonword-targets (neutral and word). Half of the critical word-targets were high category dominance (HCD) exemplars, and half were low category dominance (LCD) exemplars. Therefore, there were $12 \mathrm{HCD}$ targets and $12 \mathrm{LCD}$ targets in each of the three word-target priming conditions. Half of the 24 nonword-targets in each of the two nonword-target priming conditions were derived from HCD words and half were derived from LCD words. Of course 'dominance' is actually a pseudodistinction in this case since it is meaningless to classify nonwords in terms of word dominance. Nonetheless, in order to create the desired 24 nonwords for each of the two priming conditions, 12 were derived from HCD words and 12 were derived from LCD words. For ease of reference, these will be referred to as "HCD" nonwords and "LCD" nonwords.

Error rates for all critical word targets are listed in Table 3, and error rates for all critical nonword targets are listed in Table 4. These tables list each set of critical targets with the list versions and priming conditions in which they were presented. For example, the HCD word target "cotton" was preceded by a related prime in list versions $A$ and $B$, by a neutral prime in list versions $C$ and $D$, and by an unrelated prime in list versions $\mathrm{E}$ and $\mathrm{F}$. Initial examination of the raw data revealed an excessively high error 
Table 3. Error rates for critical word targets collapsed across list versions and priming conditions. Items removed from the analysis are marked with an asterisk.

List Versions/Priming Conditions
$\mathrm{AB}$ - Related
AB - Unrelated
AB - Neutral
CD - Neutral
CD - Related
CD - Unrelated
EF - Unrelated
EF - Neutral
EF- Related

\begin{tabular}{llllll}
\hline HCD & & HCD & & HCD \\
Targets & $\%$ errors & $\begin{array}{l}\text { Targets } \\
\text { Targets }\end{array}$ & $\%$ errors \\
COTTON & .01 & WOOL* & .03 & RAYON* & .13 \\
DOG & .01 & CAT & .01 & HORSE & .00 \\
BLUE & .00 & GREEN & .01 & RED & .01 \\
DAISY & .01 & TULIP & .01 & ROSE & .01 \\
BOMB* & .02 & KNIFE & .01 & CLUB* & .04 \\
RAPE* & .03 & THEFT* & .05 & MURDER & .00 \\
LEGS & .01 & FOOT & .01 & HEAD & .01 \\
APPLE & .00 & GRAPE & .01 & PEAR & .02 \\
TRAIN & .01 & CAR & .01 & TRUCK & .01 \\
BEANS & .01 & CARROT & .00 & CORN & .00 \\
MAPLE & .00 & PINE & .01 & OAK & .00 \\
PANTS & .01 & SHOES & .01 & SHIRT & .00 \\
& & & & & \\
LCD & & LCD & & LCD & \\
Targets & $\%$ errors & Targets & $\%$ errors & Targets & $\%$ errors \\
& & & & & \\
MADRAS* & .81 & FELT* & .11 & TWEED & .19 \\
FOX & .00 & ELK & .06 & MOOSE & .01 \\
AQUA & .05 & MAUVE & .10 & TAN & .04 \\
POPPY & .09 & LILAC & .06 & POSY* & .57 \\
ROCK & .00 & STICK & .01 & WHIP & .01 \\
LIBEL* & .73 & FRAUD & .10 & BIGAMY* & .45 \\
BACK & .06 & BONE & .03 & CHIN & .14 \\
PRUNE & .07 & BERRY & .02 & LIME & .02 \\
CANOE & .01 & JET & .01 & BUGGY & .06 \\
ONION & .02 & TURNIP & .01 & LEAK & .09 \\
HOLLY & .05 & PALM & .01 & ASH & .03 \\
SMOCK & .18 & PARKA* & .37 & BOOTS & .01 \\
\hline & & & & & \\
\hline
\end{tabular}

Reproduced with permission of the copyright owner. Further reproduction prohibited without permission. 
Table 4. Error rates for critical nonword targets collapsed across list versions and priming conditions. Items removed from the analysis are marked with an asterisk.

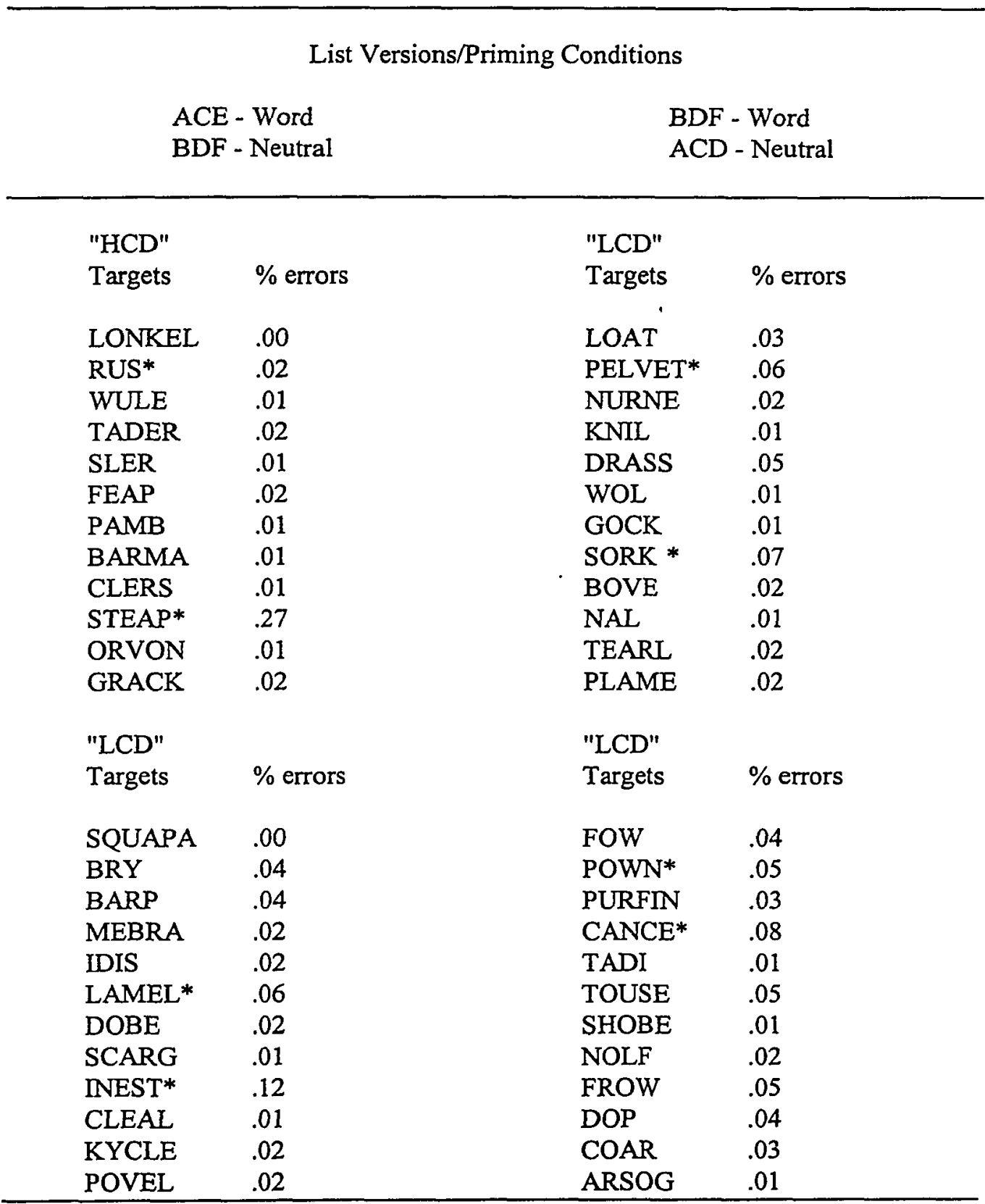


rate for some of the target stimuli, especially for LCD targets. For example, the LCD target "madras" was responded to in error by $81 \%$ of the subjects and "libel" was responded to in error by $73 \%$ of the subjects (across the three priming conditions). Such high error rates suggest that these targets were simply not in the lexicon of a large number of subjects. In order to remove such items from the analysis, the number of critical targets for each dominance/priming condition was reduced from 12 to 10 by removing the two items that incurred the greatest number of errors across priming conditions and across stimulus list versions. For example, of the twelve LCD target words that were preceded by a related prime in lists $A$ and $B$, a neutral prime in lists $C$ and D, and an unrelated prime in lists $E$ and F, "madras" and "libel" incurred the greatest number of errors, and therefore these two items were removed from further analysis. In this manner, an equal number of target stimuli were maintained across all dominance/priming conditions and list versions. Those targets removed from further analysis are marked with an asterisk in Tables 3 and 4.

\section{Word-target data}

\section{$\underline{\text { Reaction Time Analysis }}$}

The mean reaction times (RTs) for correct responses to word targets in each of the three priming conditions (related, unrelated, and neutral), the two dominance conditions (HCD and LCD), and each SOA condition, are presented in Table 5. Differences between these means are reported in Table 6 as facilitation (neutral minus related), inhibitory (neutral minus unrelated) and overall priming effects (unrelated minus related). Before these mean values were calculated, all RTs $\geq 2000 \mathrm{~ms}$ were 
Table 5. Experiment 1 reaction time word-target data summary. Mean reaction times in milliseconds to word-target pairs in all dominance, priming ( $R=$ related, $U=$ unrelated, $N=$ neutral, and SOA conditions.

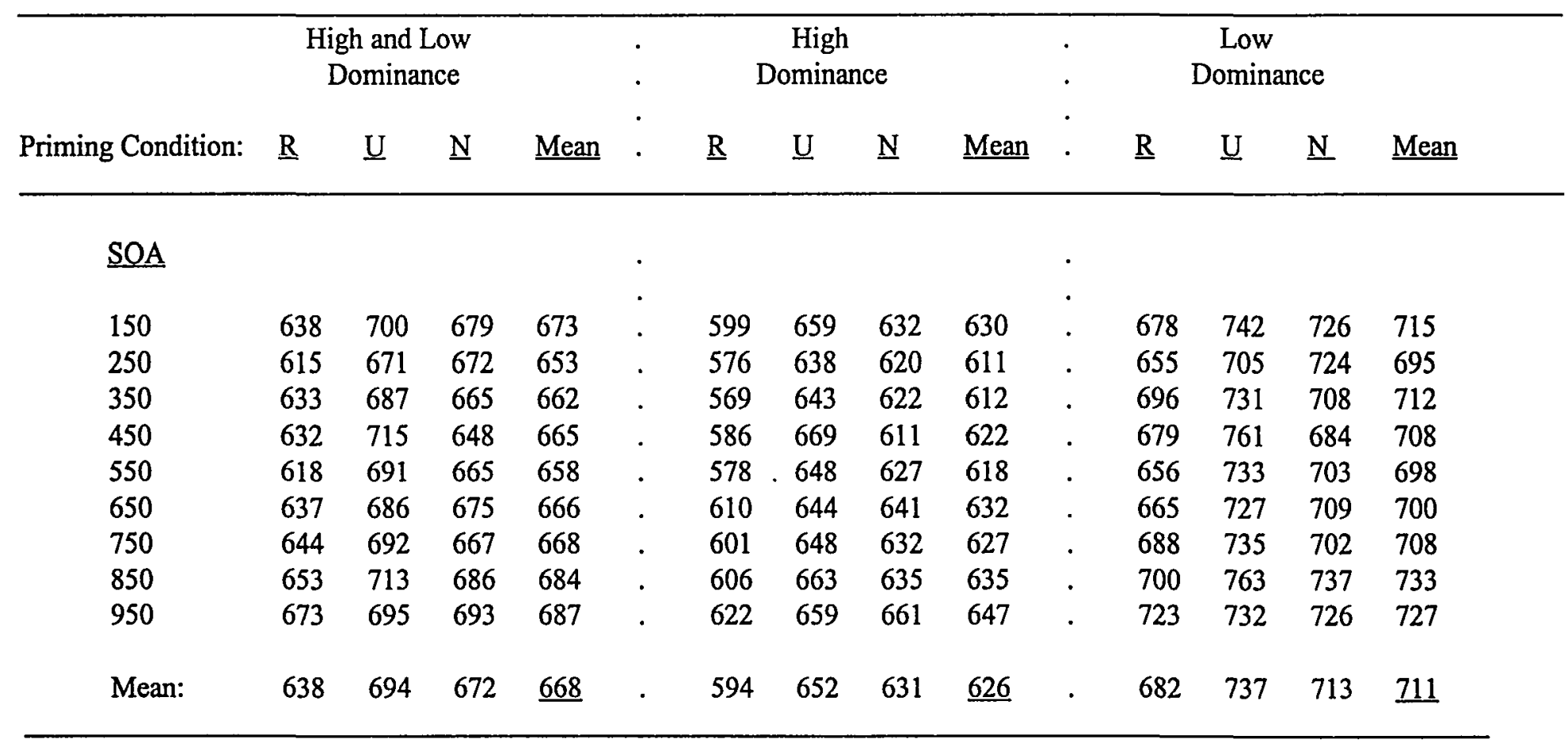


Table 6. Experiment 1 reaction time priming effects summary. Priming effects representing the differences between mean reaction times for each of the three priming conditions are presented for each SOA and dominance condition. (Facil=neutral minus related, Inhib=neutral minus unrelated, and Overall=unrelated minus related).

\begin{tabular}{|c|c|c|c|c|c|c|c|c|c|c|c|}
\hline \multirow[b]{2}{*}{ Priming Effects: } & \multicolumn{3}{|c|}{$\begin{array}{l}\text { High and Low } \\
\text { Dominance }\end{array}$} & \multirow{2}{*}{. } & \multicolumn{3}{|c|}{$\begin{array}{c}\text { High } \\
\text { Dominance }\end{array}$} & \multirow{2}{*}{$\begin{array}{l}. \\
. \\
.\end{array}$} & \multicolumn{3}{|c|}{$\begin{array}{c}\text { Low } \\
\text { Dominance }\end{array}$} \\
\hline & Facil & $\underline{\text { Inhib }}$ & Qverall & & $\underline{\text { Facil }}$ & Inhib & Qverall & & Facil & $\underline{\text { Inhib }}$ & Overall \\
\hline SOA & & & & & & & & & & & \\
\hline 150 & $+40^{\mathrm{a}}$ & -22 & $+62^{\mathrm{a}}$ & . & $+33^{b}$ & -27 & $+60^{\mathrm{a}}$ & . & $+47^{d}$ & -16 & $+64^{a}$ \\
\hline 250 & $+57^{\mathrm{a}}$ & +01 & $+56^{\mathrm{a}}$ & . & $+44^{a}$ & -17 & $+62^{a}$ & . & $+69^{a}$ & +18 & $+51^{d}$ \\
\hline 350 & $+32^{b}$ & -22 & $+54^{a}$ & . & $+52^{d}$ & -21 & $+74^{\mathrm{a}}$ & $\cdot$ & +12 & -23 & +35 \\
\hline 450 & +15 & $-67^{a}$ & $+83^{a}$ & . & +25 & $-58^{a}$ & $+83^{a}$ & $\cdot$ & +06 & $-77^{\mathrm{a}}$ & $+83^{a}$ \\
\hline 550 & $+47^{b}$ & -25 & $+72^{a}$ & $\cdot$ & $+49^{b}$ & -21 & $+70^{\mathrm{a}}$ & . & +47 & -30 & $+77^{a}$ \\
\hline 650 & $+38^{c}$ & -11 & $+48^{a}$ & $\cdot$ & +31 & -04 & +34 & ${ }^{\circ}$ & +44 & -18 & $+63^{b}$ \\
\hline 750 & +22 & -25 & $+47^{a}$ & . & +31 & -16 & $+47^{b}$ & • & +14 & -33 & $+47^{a}$ \\
\hline 850 & $+33^{d}$ & $-27^{\mathrm{h}}$ & $+60^{a}$ & $\cdot$ & $+30^{h}$ & -28 & $+57^{\mathrm{b}}$ & $\cdot$ & +37 & -26 & $+63^{a}$ \\
\hline 950 & +20 & -02 & $+22^{-}$ & . & +38 & +02 & $+36^{-}$ & $\cdot$ & +03 & -06 & +09 \\
\hline Mean: & $+34^{c}$ & $-22^{f}$ & $+56^{a}$ & . & +37 & -21 & +58 & . & +31 & -23 & +54 \\
\hline
\end{tabular}

Note: Priming effects without superscript are not significant by either analysis. a Significant at the .01 level by both the subject and item analysis. 'Significant at the .05 level by both the subject and item analysis. "Significant at the .01 level by the subject analysis and at the .05 level by the item analysis. 'Significant at the .05 level by the subject analysis and at the .01 level by the item analysis. ${ }^{\circ}$ Significant at the .05 level by the subject analysis but not significant by the item analysis. 'Significant at the .01 level by the subject analysis but not significant by the item analysis. ${ }^{8}$ Significant at the .01 level by the item analysis but not significant by the subject analysis. "Significant at the .05 level by the item analysis but not significant by the subject analysis. -Main effect for priming was significant, but individual differences between means did not reach significant level with Tukey post hoc analysis. 
discarded from the reaction time analysis and treated as errors. These long latencies occurred on $.02 \%$ of the correct word target responses. To further reduce variability in the data, each subject's data was cleaned individually. Missing values (errors) and extreme values (RTs +/-2 SD from the subject's mean per condition) were replaced with the subject's mean RT for that condition. Similar methods for reducing variability in reaction time data have been reported by other investigators (de Groot, 1986; Neely, Keefe, \& Ross, 1989; Forster \& Shen, 1996, Hagoort, 1997; Katz, 1988; Ostrin \& Tyler, 1993). Missing value replacements accounted for $3.14 \%$ of the data and extreme value replacements accounted for $3.97 \%$ of the data. These error rates are similar to those reported in the literature (Becker, 1980; de Groot, 1986, Lorch, Balota \& Stamm, 1986).

A separate $9(\mathrm{SOA}) \times 3$ (prime) $\times 2$ (dominance) analysis of variance was performed with subject means (averaged across targets) and item means (averaged across subjects). For the subject mean analysis, SOA was treated as a between subjects factor, and prime and dominance served as within subjects factors. For the item mean analysis dominance served as a between items factor and SOA and prime served as within items factors.

The main effect for prime was reliable on both analyses $\left[F_{1}(2,306)=82.44, p<\right.$ $\left..001 ; F_{2}(2,116)=79.34, p<.001\right]$. The difference between the mean RTs for each of the three priming conditions was examined using a Tukey post hoc analysis. These mean values (averaged across SOA and dominance conditions) are shown at the bottom of the first panel of Table 5. The difference values are shown at the bottom of the first panel of Table 6. As shown in Table 6, the mean RT to word targets was 34 ms faster in 
the related condition than in the neutral condition (facilitation), 22 seconds slower in the unrelated condition than in the neutral condition (inhibition), and $56 \mathrm{~ms}$ faster in the related condition than in the unrelated condition (overall priming). For the subject mean analysis all three of these differences were significant [Tukey HSD cv $=18.21,<.01]^{2}$ For the item mean analysis, the overall priming [Tukey HSD cv $=40.56, \mathrm{p}<.01$ ] and facilitation effects [Tukey HSD cv $=32.12, \mathrm{p}<.05$ ] were reliable, however the $-22 \mathrm{~ms}$ inhibition effect was not statistically significant.

The main effect for dominance was also reliable on both analyses $\left[F_{1}(1,153)=\right.$ 506.37, $\left.\mathrm{p}<.001 ; \mathrm{F}_{2}(1,58)=79.51, \mathrm{p}<.001\right]$. This analysis indicates that, averaged across SOA and prime conditions, the $85 \mathrm{~ms}$ difference between the $626 \mathrm{~ms}$ mean RT to HCD targets, and the $711 \mathrm{~ms}$ mean RT to LCD targets, was statistically reliable. These mean values are shown at the bottom of the last two panels of Table 5 .

The main effect for SOA was reliable only on the item mean analysis $\left[F_{1}(8,153)\right.$ $\left.=.225, \mathrm{p}<.99 ; \mathrm{F}_{2}(8,464)=8.89, \mathrm{p}<.001\right]$. The great disparity between these two $\mathrm{F}$ values is likely due to the larger degree of variability observed across subjects (overall standard deviation $(\mathrm{SD})=99.89$ ) than across items (overall $\mathrm{SD}=62.66$ ). The mean $\mathrm{RT}$ for each SOA condition, averaged across prime and dominance conditions, is shown in the last column of the first panel of Table 5. Tukey post hoc analyses of the difference between mean RTs (across items) for each SOA condition indicated that the mean RT obtained in the 950 condition ( $687 \mathrm{~ms}$ ) was reliably slower than the mean RT obtained in the 550 condition $(658 \mathrm{~ms})$ and the mean RT obtained in the 250 condition ( $653 \mathrm{~ms}$ ) [Tukey HSD cv $=28.89, \mathrm{p}<.05$, and Tukey HSD cv $=33.43, \mathrm{p}<.01$, respectively]. 
Also, the mean RT obtained in the 850 condition $(684 \mathrm{~ms})$ was reliably slower than that obtained in the 250 condition. No other differences were significant.

The interaction between prime and SOA (averaged across dominance conditions) approached significance with the subject mean analysis $\left[F_{1}(16,306)=1.64\right.$, $\mathrm{p}<.06]$, and was significant with the item mean analysis $\left[F_{2}(16,928,=1.66, p<.05]\right.$. A one-way analysis of variance for subject and item mean RTs obtained in the three priming conditions was performed separately for each SOA condition as a test of simple main effects to determine the nature of this interaction (Maxwell \& Delaney, 1990). Relevant statistics from this analysis are reported in Table 7. Both item and subject mean analyses yielded a main effect for priming in all but the 950 SOA condition. A Tukey post hoc analysis of the difference between the mean RTs for each of the three priming conditions was performed for each SOA condition. These differences are reported in the first panel of Table 6 as the facilitation, inhibition and overall priming effects. Tukey HSD critical difference values are reported in Table 7. Because the statistical significance of the priming effects reported in these tables was determined by separate analyses for each SOA group, the magnitude of the effect required to meet the .05 or greater level of significance varied between the 9 SOA groups. As indicated in the first panel of Table 6, the overall priming effect (unrelated minus related) was reliable in all but the 950 SOA condition. Though some degree of facilitation (neutral minus related) was observed in each SOA condition, this effect failed to reach statistical significance in the 450,750 and 950 SOA conditions (by either the subject or item analysis). Inhibition contributed to overall priming in all but the 250 and 950 SOA 
Table 7. Results of one-way ANOVAs for mean RTs obtained in the the three priming conditions (averaged across dominance) for each SOA condition in Experiment 1.

\begin{tabular}{|c|c|c|c|c|c|c|c|c|}
\hline \multirow[b]{2}{*}{$\underline{\mathrm{SQA}}$} & \multicolumn{4}{|c|}{$\begin{array}{c}\text { Tukey CV } \\
q(3,34)\end{array}$} & & & \multicolumn{2}{|c|}{$\begin{array}{c}\text { Tukey CV } \\
\text { q }(3,118)\end{array}$} \\
\hline & \multicolumn{2}{|c|}{$E_{1}(2,34), p$ value } & .05 & .01 & \multicolumn{2}{|c|}{$\mathrm{E}_{2}(2,118), \mathrm{p}$ value } & .05 & .01 \\
\hline 150 & 13.38 & $<.001$ & 29.98 & 38.23 & 11.84 & $<.001$ & 30.71 & 38.39 \\
\hline 250 & 16.38 & $<.001$ & 28.14 & 35.87 & 16.93 & $<.001$ & 26.64 & 33.31 \\
\hline 350 & 9.60 & $<.001$ & 30.78 & 39.25 & 12.12 & $<.001$ & 26.38 & 32.97 \\
\hline 450 & 17.28 & $<.001$ & 37.00 & 47.17 & 22.15 & $<.001$ & 31.45 & 39.31 \\
\hline 550 & 9.81 & $<.001$ & 40.80 & 52.02 & 7.96 & $<.002$ & 43.61 & 54.52 \\
\hline 650 & 9.25 & $<.002$ & 29.18 & 37.20 & 5.64 & $<.006$ & 35.95 & 44.94 \\
\hline 750 & 7.53 & $<.001$ & 29.94 & 38.18 & 9.28 & $<.001$ & 25.97 & 32.47 \\
\hline 850 & 11.74 & $<.001$ & 30.64 & 39.07 & 17.77 & $<.001$ & 23.99 & 29.98 \\
\hline 950 & 1.57 & $<.224$ & $\mathrm{n} / \mathrm{a}$ & $\mathrm{n} / \mathrm{a}$ & 1.94 & $<.149$ & $\mathrm{n} / \mathrm{a}$ & $\mathrm{n} / \mathrm{a}$ \\
\hline
\end{tabular}


conditions, however this effect was only significant (by both the subject and item analyses) in the 450 SOA condition. These patterns of inhibition and facilitation are shown in Figure 1, which provides a graph of mean RTs for correct responses to word targets in the unrelated, neutral, and related priming conditions (averaged across dominance) as a function of SOA.

The interaction between dominance and SOA was not statistically significant by either the subject mean or item mean analysis $\left[F_{1}(8,153)=.745, p<.66 ; F_{2}(8,464)=\right.$ $1.626, \mathrm{p}<.12]$. As may be noted in the last two panels of Table 5, the mean RT for HCD targets (averaged across the three priming conditions) was faster than that for LCD targets in each of the nine SOA conditions.

The interaction between prime and dominance $\left[F_{1}(2,306)=.309, p<.74 ; F_{2}\right.$ $(2,116)=.261, \mathrm{p}<.78]$ was also not significant. This result, in combination with the main effect for prime, suggests that the priming effects reported for HCD and LCD targets combined (reported at the bottom of the first panel of Table 7 ) were equally significant for HCD and LCD targets (averaged across SOAs). As may be noted on the last line of the last two panels of Table 6, averaged across SOAs, the magnitude of the facilitation, inhibition, and overall priming effects was similar for HCD and LCD targets $(+37,-21,+58$ and $+31,-23,+54 \mathrm{~ms}$, respectively).

There was no significant three-way interaction between prime, dominance, and $\operatorname{SOA}\left[F_{1}(2,16,306)=.74, p<.76 ; F_{2}(2,16,116)=.591, p<.90\right]$ by either the subject mean or item mean analysis. 


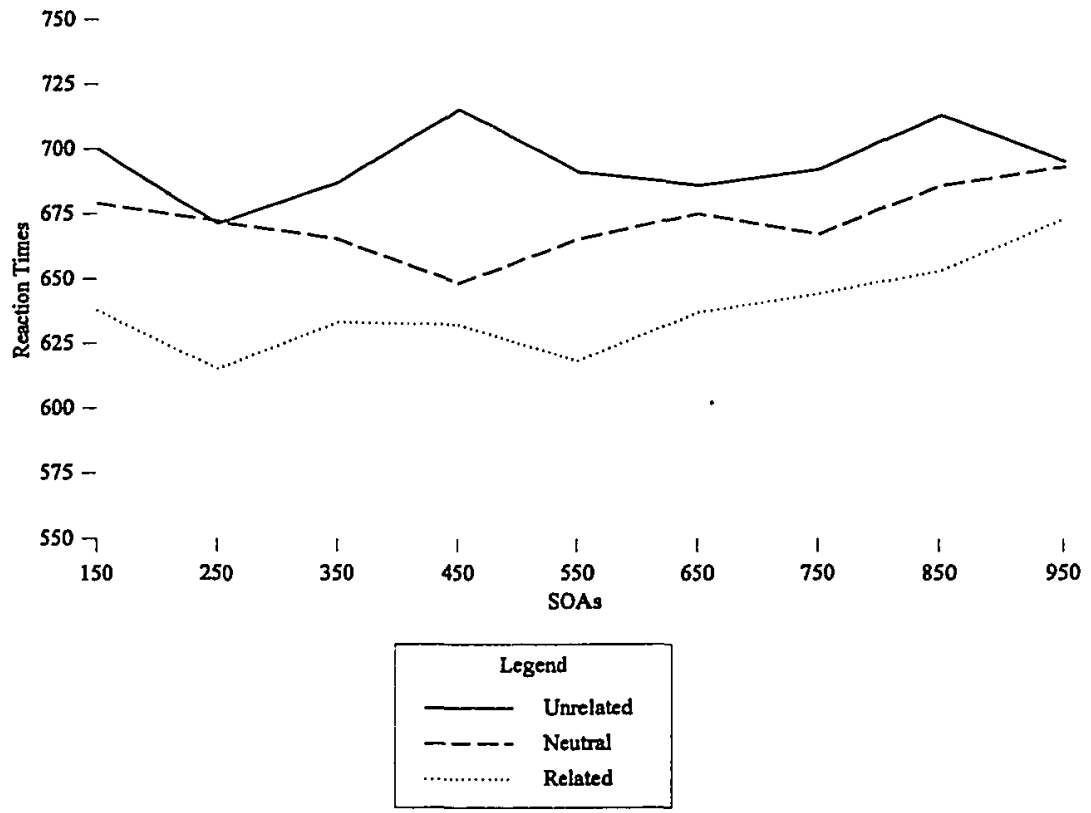

Figure 1. Mean reaction time (in milliseconds) for correct responses to word targets in the unrelated, neutral, and related priming conditions (collapsed across dominance) as a function of SOA. 
A test of simple main effects is not generally recommended in the absence of a significant interaction (Maxwell \& Delaney, 1990). Nonetheless, a separate one-way ANOVA for subject and item mean RTs obtained in the three priming conditions was performed separately for each of the individual SOA/dominance conditions in order to determine whether the facilitation, inhibition, and overall priming effects were significant in each of the individual SOA/dominance conditions. Such an analysis is justified as a test of the "existence proof" of whether priming was or was not present in each of the relevant conditions (cf. Swinney, Zurif, and Nicol, 1989, notes 2 and 4). The Neely Keefe model predicted that facilitation without inhibition would be observed in short SOA conditions, and that inhibition effects would be observed for HCD targets prior to (i.e., with a shorter SOA) than for LCD targets. The means for each SOA/dominance condition are reported in the last two panels of Table 5. For those conditions in which a main effect of priming was observed, Tukey post hoc analyses were performed to determine the significance levels of the differences between the means. Relevant statistics from these analyses are reported in Table 8. Differences between the means are reported as priming effects in the last two panels of Table 6. Significance levels are indicated by superscript. As indicated by the $\mathrm{p}$ values reported in Table 8, a main effect for prime was observed for HCD targets in all but the 650 SOA condition, by both the subject and item analyses. As noted in the second panel of Table 6, for HCD targets, the overall priming effect was significant in all but the 650 and 950 SOA conditions (by both subject and item analyses). The facilitation effect was significant by both subject and item analyses in the $150,250,350$, and $550 \mathrm{SOA}$ 
Table 8. Results of one-way ANOVAs for mean RTs obtained in the three priming conditions for high- and low-category dominance word-targets in each SOA condition in Experiment 1.

\section{High Category Dominance}

Tukey CV

$\mathrm{q}(3,34)$
Tukey CV

$q(3,58)$
$\underline{S O A} E_{i}(2,34)$, p value $\quad \underline{.05} \quad \underline{.01}$

$\begin{array}{rrr}150 & 10.14 & <.001 \\ 250 & 12.41 & <.001 \\ 350 & 9.22 & <.002 \\ 450 & 14.08 & <.001 \\ 550 & 9.47 & <.002 \\ 650 & 2.12 & <.137 \\ 750 & 3.35 & <.048 \\ 850 & 10.65 & <.001 \\ 950 & 3.58 & <.040\end{array}$

$150 \quad 10.14<.001$

$32.98 \quad 42.05$

$\begin{array}{ll}31.51 & 40.18\end{array}$

$43.63 \quad 55.63$

$39.51 \quad 50.37$

$40.90 \quad 52.15$

$\mathrm{n} / \mathrm{a} \quad \mathrm{n} / \mathrm{a}$

$\begin{array}{ll}45.89 \quad 58.52 \\ 30.64 & 39.07\end{array}$

$30.64 \quad 39.07$

$39.68 \quad 50.60$
$\mathrm{E}_{2}(2.58)$, p value $\quad .05 \quad .01$

$6.56<.004 \quad 28.22 \quad 35.52$

$13.10<.001$

$12.40<.001$

$10.07<.001$

$4.16<.021$

$1.93<.155$

$4.17<.021$

$12.01<.001$

$3.35<.043$
$21.48 \quad 26.62$

$25.91 \quad 32.61$

$32.16 \quad 40.49$

$42.50 \quad 53.50$

$\mathrm{n} / \mathrm{a} \quad \mathrm{n} / \mathrm{a}$

$40.12 \quad 50.50$

$28.08 \quad 35.35$

$40.12 \quad 50.38$

\section{Low Category Dominance}

Tukey CV

$\mathrm{q}(3,34)$
Tukey CV

$q(3,58)$

$\underline{\text { SOA }} E_{1}(2,34)$, p value $\quad \underline{.05} \quad \underline{.01} \quad E_{2}(2,58)$,p value $\quad .05 \quad \underline{.01}$

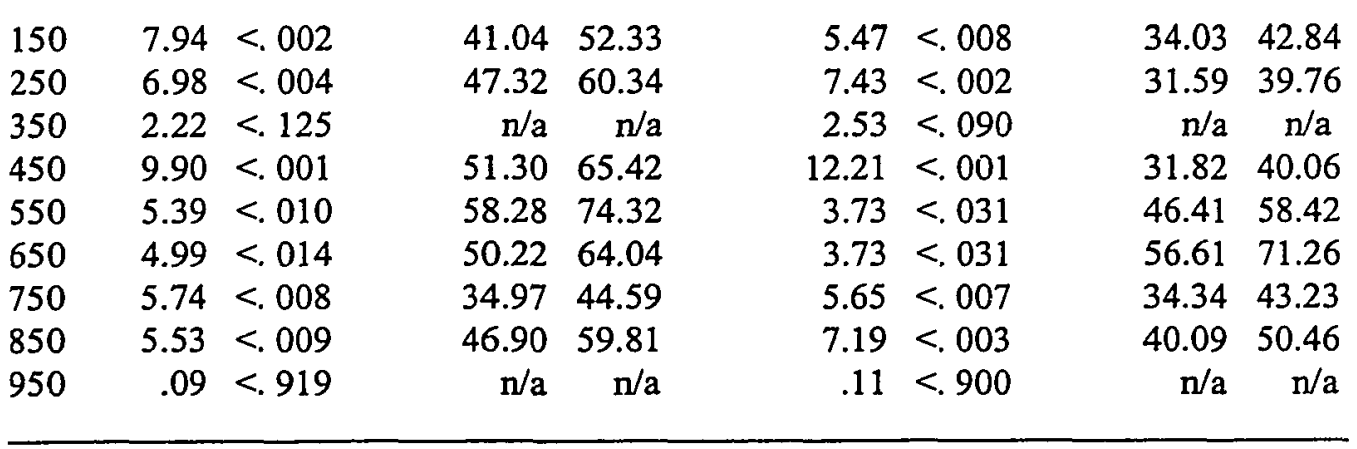


conditions. The inhibition effect was only significant in the 450 SOA condition, again by both item and subject analyses. Note that although there was a main effect for priming for HCD targets in the 950 SOA condition $(p<.040$, Table 8$)$, the $+38 \mathrm{~ms}$ facilitation effect and +36 ms overall priming effect (Table 6) only approached statistical significance with the Tukey post hoc analyses. For LCD targets, a main effect for prime was observed in all but the 350 and 950 SOA conditions, by both the subject and item analyses. As noted in the third panel of Table 6, the overall priming effect for LCD targets was significant by both subject and item analyses in all but the 350 and 950 SOA conditions. The facilitation effect for LCD targets was significant, by both analyses, only in the 150 and 250 SOA conditions. As with the HCD targets, the inhibition effect for LCD targets was only significant in the 450 SOA condition, by both subject and item analyses. The patterns of inhibition and facilitation observed across SOA conditions for the HCD and LCD targets are shown in Figures 2 and 3, respectively. Notice in both cases the relatively large amount of facilitation in the 250 SOA condition, and the relatively large amount of inhibition in the 450 SOA condition. Also notice the robust facilitation effect observed for HCD targets, but not LCD targets, in the 350 SOA condition.

Error Analysis.

The mean percentage of errors for incorrect responses to word-targets in all dominance, priming, and SOA conditions are presented in Table 9 . Table 10 presents the differences between the mean percentage of errors obtained in each of the three priming conditions. The mean percentage of errors per condition were submitted to the 


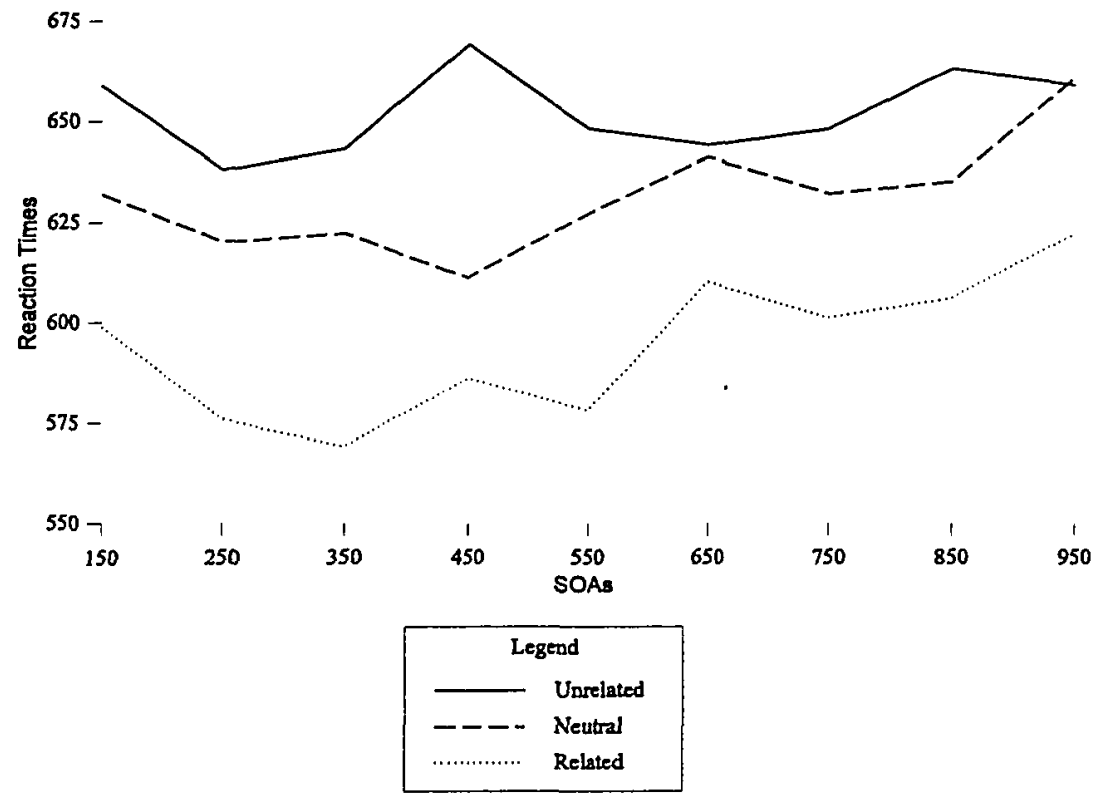

Figure 2. Mean reaction time (in milliseconds) for correct responses to HCD word targets in the unrelated, neutral, and related priming conditions (collapsed across dominance) as a function of SOA. 


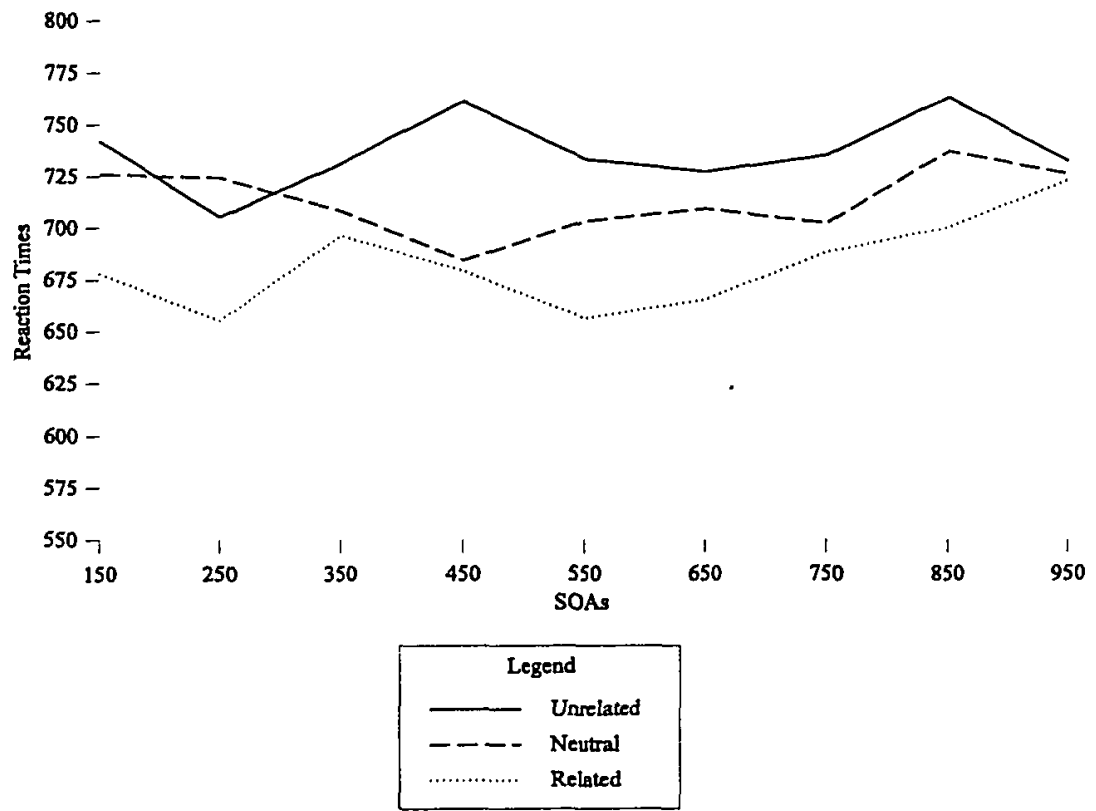

Figure 3. Mean reaction time (in milliseconds) for correct responses to LCD word targets in the unrelated, neutral, and related priming conditions (collapsed across dominance) as a function of SOA. 
Table 9. Experiment 1 error rate summary (in percentages) for word-target pairs in all dominance, priming ( $R=r e l a t e d, ~ U=u n r e l a t e d$, $\mathrm{N}=$ neutral), and SOA conditions.

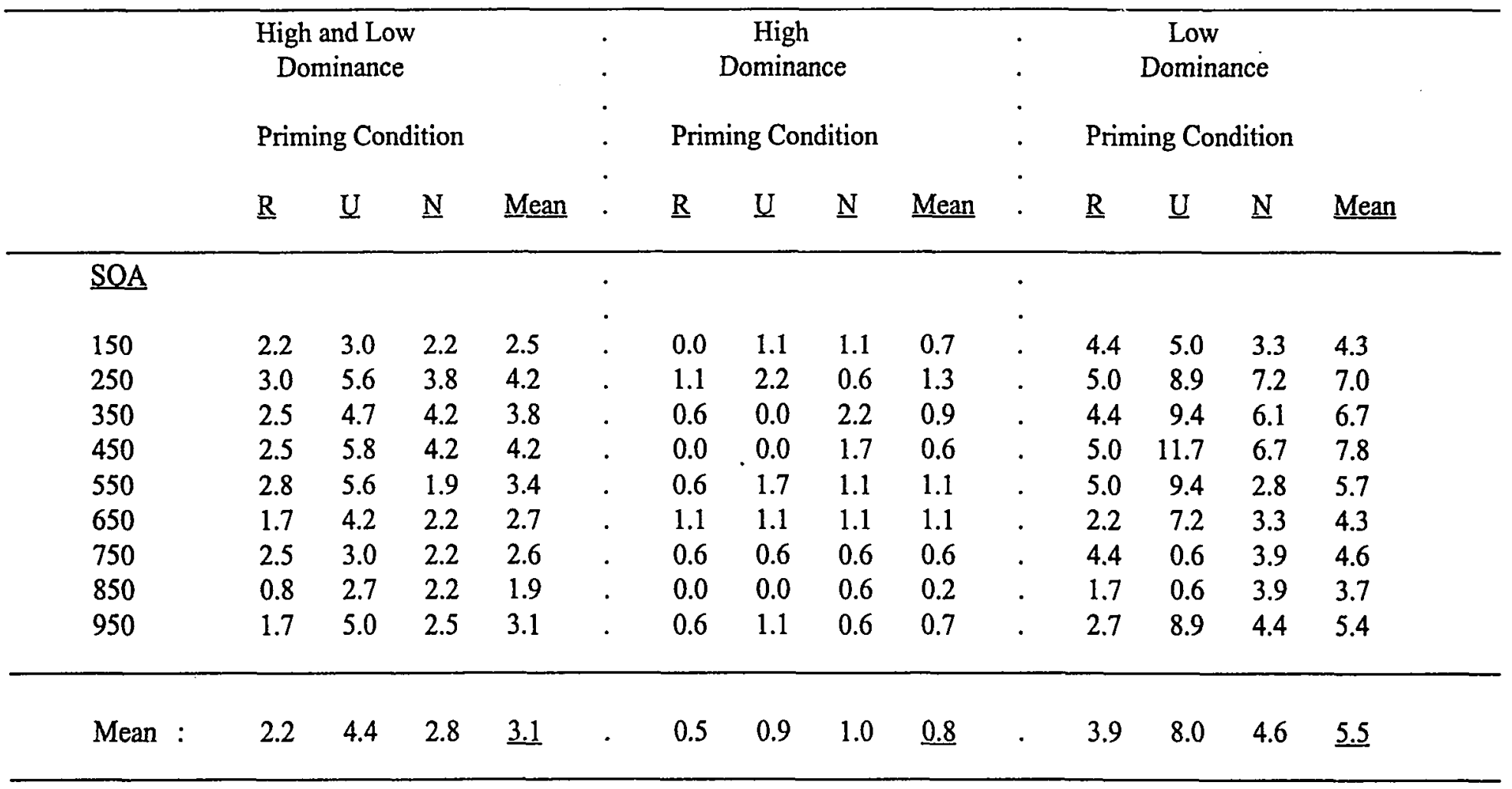


Table 10. Experiment 1 error rate priming effects summary. Priming effects for each SOA and dominance condition representing the differences between percentage of errors observed in each of the three priming conditions: Facil (neutral minus related), Inhib (neutral minus unrelated) and Overall (unrelated minus related).

\begin{tabular}{|c|c|c|c|c|c|c|c|c|c|c|c|}
\hline \multirow{2}{*}{$\begin{array}{l}\text { Priming } \\
\text { Effect: }\end{array}$} & \multicolumn{3}{|c|}{$\begin{array}{l}\text { High and Low } \\
\text { Dominance }\end{array}$} & \multirow{2}{*}{$\begin{array}{l}. \\
\cdot \\
\cdot\end{array}$} & \multicolumn{3}{|c|}{$\begin{array}{c}\text { High } \\
\text { Dominance }\end{array}$} & \multirow{2}{*}{$\begin{array}{l}\cdot \\
\cdot \\
\cdot \\
\cdot\end{array}$} & \multicolumn{3}{|c|}{$\begin{array}{c}\text { Low } \\
\text { Dominance }\end{array}$} \\
\hline & Facil & Inhib & Overall & & Facil & $\underline{\text { Inhib }}$ & Overall & & Facil & Inhib & Overall \\
\hline \multicolumn{12}{|l|}{ SOA } \\
\hline$\overline{150}$ & 0.0 & -0.8 & +0.8 & . & +1.1 & 0.0 & +1.1 & . & +1.1 & -1.7 & +0.6 \\
\hline 250 & +0.8 & -1.7 & +2.5 & . & +0.6 & -1.7 & +1.1 & . & +2.2 & -1.7 & +3.9 \\
\hline 350 & +1.7 & -0.6 & +2.2 & . & +1.7 & +2.2 & -0.6 & . & +1.7 & -3.3 & +5.0 \\
\hline 450 & +1.7 & -1.7 & +3.3 & & +1.7 & +1.7 & 0.0 & . & +1.7 & -5.0 & +6.7 \\
\hline 550 & +0.8 & -3.6 & +2.8 & . & +0.6 & -0.6 & +1.1 & . & +1.7 & -6.7 & +4.4 \\
\hline 650 & +0.6 & -1.9 & +2.5 & . & 0.0 & 0.0 & 0.0 & . & +1.1 & -3.9 & +5.0 \\
\hline 750 & +0.3 & -0.8 & +0.6 & . & 0.0 & -1.2 . & 0.0 & . & +0.6 & -1.7 & +1.1 \\
\hline 850 & +1.4 & -0.6 & +1.9 & . & +0.6 & +0.6 & 0.0 & . & +2.2 & -1.7 & +3.9 \\
\hline 950 & +0.8 & -2.5 & $+3.3^{b}$ & . & 0.0 & -0.6 & +0.6 & . & +1.7 & -4.4 & +6.1 \\
\hline$\overline{\text { Sum: }}$ & +0.6 & $-1.6^{f}$ & $+2.2^{e}$ & . & +0.5 & +1.9 & +3.7 & . & +0.7 & $-3.3^{a}$ & $+4.1^{\mathrm{a}}$ \\
\hline
\end{tabular}

Note: Significance levels are based on Tukey post hoc analyses using mean error rates per condition. Priming effects without superscript are not significant by either analysis. " Significant at the .01 level by both the subject and item analysis. 'Significant at the .05 level by both the subject and item analysis. 'Significant at the .01 level by the subject analysis and at the .05 level by the item analysis. "Significant at the .05 level by the subject analysis and at the .01 level by the item analysis. 'Significant at the .01 level by the subject analysis but not significant by the item analysis. ' Significant at the .05 level by the subject analysis but not significant by the item analysis. ${ }^{8}$ Significant at the .01 level by the item analysis but not significant by the subject analysis. "Significant at the .05 level by the item analysis but not significant by the subject analysis. 
same statistical analyses as were the reaction time data. The $9(\mathrm{SOA}) \times 3$ (prime) $\mathrm{x}$ 2(dominance) ANOVA yielded a main effect for prime type $\left[F_{1}(2,306)=14.515, p<\right.$ $\left..001 ; F_{2}(2,116)=10.213, p<.001\right]$, by both subject and item analyses. Results of Tukey post hoc analyses are indicated in Table 10. Across SOA and dominance conditions, $0.6 \%$ fewer errors were made in the related condition than in the neutral condition, $1.6 \%$ more errors were made in the unrelated condition than in the neutral condition, and $2.2 \%$ more errors were observed in the unrelated condition than in the related condition. The overall priming effect and the inhibitory effect were significant by the subject [Tukey HSD $c v=1.4, p<.05 ; \mathrm{cv}=1.7, \mathrm{p}<.01$ ], but not the item mean [Tukey HSD cv $=3.8, \mathrm{p}<.05$ ] analysis. The facilitation effect was not significant by either analysis.

The main effect for dominance was also significant $\left[F_{l}(1,153)=121.458, p<\right.$ $\left..001 ; F_{2}(1,58)=23.941, p<.001\right]$ by both subject and item mean analyses. The mean percentage of error responses to $\mathrm{HCD}$ targets and to LCD targets are shown at the bottom of the last two panels of Table 9. The main effect for dominance indicates that significantly fewer errors were observed in response to $\mathrm{HCD}$ targets $(.8 \%)$ than in response to LCD targets (5.5\%). These results are consistent with the reaction time data, indicating that responses were faster and more accurate to HCD word-targets than to LCD word-targets, and to word-targets preceded by a related versus a neutral versus an unrelated target.

The main effect for SOA was reliable only on the item mean analysis $\left[F_{1}(8,153)\right.$ $\left.=1.301, \mathrm{p}<.25 ; \mathrm{F}_{2}(8,464)=2.663, \mathrm{p}<.01\right]$. Although overall reaction times were 
significantly slower in the two longest SOA conditions, the greatest number of errors did not occur in these conditions. This suggests the possibility of a slight speed for accuracy tradeoff, however none of the Tukey post hoc [Tukey HSD cv $=3.8, \mathrm{p}<.05$ ] tests examining the difference in error rates between item means in the various SOA conditions were significant. As noted in Table 9, the highest overall error rates occurred in the 250,350 , and 450 SOA conditions.

The interaction between dominance and SOA was not statistically significant by either the subject mean or item mean analysis $\left[F_{1}(8,153)=1.08, p<.38 ; F_{2}(8,464)=\right.$ $1.90, \mathrm{p}<.06]$, although it approached significance with the latter analysis.. As may be noted in Table 9, fewer errors were observed in response to HCD compared to LCD targets in each of the individual SOA conditions. This result is consistent with the reaction time data and indicates that responses were faster and more accurate to $\mathrm{HCD}$ compared to LCD targets, overall, and within each individual SOA condition.

The prime by SOA interaction was not significant by either the subject mean or item mean analysis $\left[F_{1}(16,306)=.572, p<.91 ; F_{2}(16,464,=.60, p<.89]\right.$. The most notable aspect of this result is that the error data, but not the reaction time data, suggest some degree of priming did occur in the 950 SOA condition. As shown in the first panel of Table 9, for the 950 SOA condition, the fewest number of errors were observed in the related condition ( $1.7 \%$ ), the greatest number of errors occurred in the unrelated condition ( $5.0 \%$ ), and half as many errors occurred in the neutral condition $(2.5 \%)$ as did in the unrelated condition. A one-way ANOVA for mean error rates observed in the three priming conditions in the 950 SOA condition yielded a main effect for prime by 
both the subject and item mean analysis $\left[F_{1}(2,34)=4.51, p<.019 ; F_{2}(2,116)=3.83, p\right.$ $<.025]$. Results of the Tukey post hoc analysis are reported in Table 10. Only the overall priming effect reached statistical significance, by both the subject and the item analyses [Tukey HSD $\mathrm{cv}=2.8 \mathrm{p}<.05, \mathrm{cv}=3.1 \mathrm{p}<.05$, respectively]. Note, however, that the $-2.5 \%$ inhibition effect approached significance with the subject analysis.

In contrast to the reaction time data analysis, the error analysis revealed a significant interaction between prime and dominance $\left[F_{1}(2,306)=12.87, p<.001 ; F_{2}\right.$ $(2,116)=8.52, \mathrm{p}<.001]$. To examine this interaction, separate one-way ANOVAs of the mean percentage of errors obtained in each priming condition (averaged across SOA conditions) were performed for each of the two dominance conditions. These analyses revealed a significant main effect for priming only in the $\operatorname{LCD}$ condition $\left[F_{1}(2,322)=\right.$ $\left.15.88, p<.001 ; F_{2}(2,58)=10.09, p<.001\right]$. As shown at the bottom of the last panel of Table 9, for LCD word targets, $8.0 \%$ errors occurred in the unrelated condition, compared to $3.9 \%$ errors in the related condition, and $4.6 \%$ errors in the neutral condition. As reported at the bottom of the last panel of Table 10 , the $-3.3 \%$ inhibition effect and the $+4.1 \%$ overall priming effect were significant by both the subject and item mean analysis [Tukey $\mathrm{HSD}_{1} \mathrm{cv}=1.8 \mathrm{p}<.01$, Tukey $\mathrm{HSD}_{2} \mathrm{cv}=3.0 \mathrm{p}<.01$, respectively]. The .7\% facilitation effect was not significant. These results indicate that, averaged across SOA conditions, a significantly greater number of errors were observed in response to LCD word targets preceded by unrelated primes compared to those preceded by related or neutral primes. The failure to find a main effect for priming for the HCD condition is most likely due to the relatively few errors that 
occurred in response to $\mathrm{HCD}$ targets (total $=39,0.8 \%$ ). Nonetheless, as may be observed in Table 9, the fewest errors occurred in the related priming condition, as would be expected.

As with the reaction time analysis, there was no significant three-way interaction between prime, dominance, and SOA $\left[F_{1}(2,16,306)=.737, p<.76 ; F_{2}(2,16,116)=\right.$ $.591, \mathrm{p}<.90]$ by either the subject mean or item mean analysis.

In summary, across the entire data set, as well as in each individual SOA and dominance condition, responses to HCD word targets were significantly faster and more accurate than responses to LCD word targets. In addition, responses to word targets were faster and more accurate when the target word was preceded by a related prime compared to when the target word was preceded by an unrelated prime. This overall priming effect was observed both within and across individual SOA and dominance conditions, with few exceptions. A much less robust result was the degree to which facilitation and inhibition contributed to the overall priming effect in each of these conditions. Facilitation effects reached statistical significance in all but the 450,750 , and 950 SOA conditions, however, a significant degree of inhibition was only observed in the 450 SOA condition.

\section{Nonword target data}

\section{Reaction Time Analysis}

Mean RTs for correct responses to nonword targets in each of the two priming conditions (word, neutral), in each of the two pseudodominance conditions ("HCD", 
"LCD"), and each of the SOA condition are presented in Table 11. This table also displays the nonword facilitation effect (NWF), which is the difference between mean RTs obtained in the neutral minus the mean RTs obtained in the word priming condition. As with the word RT data, these mean values were calculated after all RTs $\geq$ $2000 \mathrm{~ms}$ were converted to errors, and missing values and extreme values were replaced with each subject's mean RT per condition. A total of $49(0.8 \%)$ of RTs $\geq 2000 \mathrm{~ms}$ were converted to errors. Missing value replacements accounted for $2.7 \%$ of the data and extreme value replacements accounted for $4.0 \%$ of the data.

As shown in Table 11, the overall mean RT (averaged across SOA, pseudodominance, and priming conditions) to nonword targets was $775 \mathrm{~ms}$, which was $107 \mathrm{~ms}$ slower than the $668 \mathrm{~ms}$ overall mean RT to word targets (see Table 5). The overall mean RT (averaged across SOA and priming conditions) to "HCD" nonword targets was $767 \mathrm{~ms}$, which was $16 \mathrm{~ms}$ faster than the 783 ms mean RT to "LCD" nonword targets. Since the pseudodistinction between nonwords derived from $\mathrm{HCD}$ words and those derived from LCD words was expected to be irrelevant, pseudodominance was considered as a factor in the statistical analyses of these data, in order to determine if this $16 \mathrm{~ms}$ difference was significant. A separate 9 (SOA) $\times 2$ (prime) $\times 2$ (pseudodominance) ANOVA was performed for subject means and item means. For the subject mean analysis, SOA was treated as a between subjects factor, and prime and dominance served as within subjects factors. For the item mean analysis, dominance served as the between items factor, and SOA and prime served as within items factors. 
Table 11. Mean reaction times in milliseconds to nonword-target pairs in all pseudodominance ("High", "Low"), priming (word prime, neutral prime) and SOA conditions. The nonword facilitation effect (NWF) represents the difference between RTs obtained in the neutral minus the word priming condition.

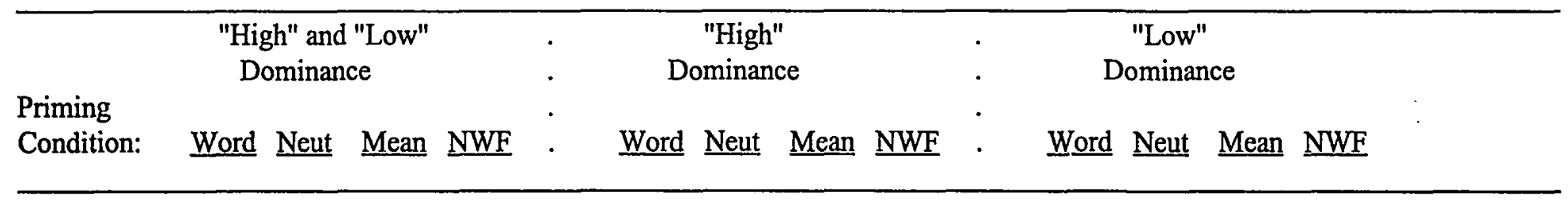

\begin{tabular}{llllllllllllllll}
\hline SOA & & & & &. & & & & & &. & & & & \\
150 & 808 & 797 & 802 & -11 &. & 780 & 793 & 787 & +13 &. & 835 & 800 & 817 & -35 \\
250 & 746 & 769 & 758 & +23 &. & 741 & 750 & 745 & +09 &. & 751 & 789 & 770 & +38 \\
350 & 726 & 730 & 728 & +05 &. & 722 & 730 & 728 & +12 &. & 730 & 727 & 729 & -02 \\
450 & 785 & 786 & 785 & -01 &. & 766 & 789 & 777 & +24 &. & 805 & 782 & 793 & -23 \\
550 & 760 & 773 & 766 & +13 &. & 744 & 757 & 750 & +13 &. & 776 & 789 & 782 & +13 \\
650 & 757 & 770 & 764 & +13 &. & 741 & 780 & 760 & +39 &. & 774 & 761 & 767 & -14 \\
750 & 780 & 798 & 789 & +18 &. & 762 & 791 & 777 & +29 &. & 799 & 804 & 802 & +06 \\
850 & 753 & 785 & 765 & +24 &. & 739 & 769 & 754 & +30 &. & 767 & 785 & 776 & +18 \\
950 & 828 & 805 & 816 & -22 &. & 841 & 809 & 825 & -32 &. & 815 & 801 & 816 & -14 \\
& & & & & & & & & & & & & & \\
Mean: & 771 & 778 & $\mathbf{7 7 5}$ & +07 &. & 760 & 775 & $\underline{767}$ & +16 &. & 783 & 782 & $\underline{783}$ & -02 \\
\hline
\end{tabular}


The main effect for pseudodominance was significant with the subject mean analysis $\left[F_{1}(1,153)=8.969, p<.004\right]$, but only marginal with the item mean analysis $\left.F_{2}(1,38)=3.106, p<.09\right]$. These results suggest that the $16 \mathrm{~ms}$ difference between the mean reaction time to "HCD" targets and the mean reaction time to "LCD" targets was statistically reliable. Averaged across priming and SOA conditions, subjects were reliably faster in their responses to nonwords derived from $\mathrm{HCD}$ words than to nonwords derived from LCD words.

The item analysis, but not the subject analysis, yielded a significant main effect for $\operatorname{SOA}\left[\mathrm{F}_{1}(8,153)=.536, \mathrm{p}<.81, \mathrm{~F}_{2}(8,304)=21.754, \mathrm{p}<.001\right]$, and a significant pseudodominance by SOA interaction $\left[F_{1}(8,153)=1.049, p<.41, F_{2}(8,304)=1.977\right.$, $\mathrm{p}<.05]$. Figure 4 displays the mean reaction time for correct responses to "HCD" and "LCD" nonword targets as a function of SOA. As this figure shows, RTs to both "LCD" and "HCD" nonword targets decrease rather sharply between the 150 SOA condition and the 350 SOA condition, increase sharply in the 450 SOA condition, then show a general (though certainly not linear) increase between the 450 SOA condition and the 950 SOA condition. RTs to "LCD" nonword targets are slower than RTs to "HCD" nonword targets in all but the 350 and 950 SOA conditions. The fact that these results were significant only with the item analysis likely reflects the much larger variability observed across subjects (overall $\mathrm{SD}=147.26$ ) than across items (overall $\mathrm{SD}=28.59$ )

The main effect for prime was not reliable on either analysis $\left[F_{1}(1,153)=\right.$ $\left.2.011, \mathrm{p}<.16 ; \mathrm{F}_{2}(1,38)=1.616, \mathrm{p}<.22\right]$. This result indicates that the $7 \mathrm{~ms}$ difference 
between RTs obtained in the word versus the neutral priming conditions (averaged across SOAs and pseudodominance conditions) was not statistically reliable.

Furthermore, prime did not interact with $\operatorname{SOA}\left[F_{1}(8,153)=1.183, p<.313 ; F_{2}(8,304)\right.$ $=.809, \mathrm{p}<.60]$, or with pseudodominance $\left[F_{1}(1,153)=2.771, \mathrm{p}<.10 ; \mathrm{F}_{2}(1,38)=\right.$ $2.347, \mathrm{p}<.14$ ], by either analysis. Nor was there a three way interaction between prime, pseudodominance, and SOA by either analysis $\left[F_{1}(1,8,153)=.062, p<.47 ; F_{2}\right.$ $(1,8,304)=.695, \mathrm{p}<.70]$. These results suggest that mean RTs to nonword targets preceded by word primes were not significantly different than mean RTs to nonword targets preceded by neutral primes, in any condition (i.e. for "HCD" or "LCD" nonword targets, separately or combined, nor across or within individual SOA conditions). Nonetheless, results reported in the last line of the last two panels of Table 11 suggests a $16 \mathrm{~ms}$ trend (overall) for subjects to respond faster to nonwords preceded by a word prime (rather than a neutral prime) when the nonword targets were derived from HCD words. This trend was not observed with nonword targets derived from LCD words. Error analysis.

The mean error rates for incorrect responses to nonword targets are reported in Table 12. These means were submitted to the same statistical analyses as were the reaction time data. These analyses yielded the same results as the RT data analysis with two exceptions. First, whereas both the subject mean and the item mean analysis of error rates yielded a significant $\mathrm{SOA} x$ pseudodominance interaction $\left[F_{1}(8,153)=\right.$ $3.262, \mathrm{p}<.003 ; \mathrm{F}_{2}(8,304)=3.142, \mathrm{p}<.003$ ], this interaction was only significant with the item mean analysis of the reaction time data. Also, whereas only the subject means 
Table 12. Mean error rates (in percentages) for nonword target pairs in all pseudodominance ("High", "Low"), priming (word prime, neutral prime) and SOA conditions. The nonword facilitation effect (NWF) represents the difference between RTs obtained in the neutral minus the word priming condition.

\begin{tabular}{|c|c|c|c|c|c|c|c|c|c|c|c|c|}
\hline \multirow{2}{*}{$\begin{array}{l}\text { Priming } \\
\text { Condition: }\end{array}$} & \multicolumn{4}{|c|}{$\begin{array}{l}\text { "High" and "Low" } \\
\text { Dominance }\end{array}$} & \multicolumn{4}{|c|}{$\begin{array}{c}\text { "High" } \\
\text { Dominance }\end{array}$} & \multicolumn{4}{|c|}{$\begin{array}{c}\text { "Low" } \\
\text { Dominance }\end{array}$} \\
\hline & Word & $\underline{\text { Neut }}$ & Mean & $\underline{\text { NWF }}$ & Word & Neut & Mean & $\underline{\text { NWF }}$ & Word & Neut & Mean & NWF \\
\hline SOA & & & & & & & & & & & & \\
\hline 150 & 2.8 & 4.4 & 3.6 & +1.7 & 1.7 & 3.4 & 2.8 & +2.2 & 3.9 & 5.0 & 4.4 & +1.1 \\
\hline 250 & 0.8 & 2.5 & 1.7 & +1.7 & 1.7 & 2.8 & 2.2 & +1.1 & 0.0 & 2.2 & 1.1 & +2.2 \\
\hline 350 & 2.2 & 0.6 & 1.4 & -1.7 & 0.6 & 0.6 & 0.6 & +7.7 & 3.9 & 0.6 & 2.2 & -3.3 \\
\hline 450 & 5.3 & 5.0 & 5.1 & -2.8 & 2.2 & 1.1 & 1.7 & -1.1 & 8.3 & 8.9 & 8.6 & +0.6 \\
\hline 550 & 2.5 & 2.8 & 2.6 & +2.8 & 0.6 & 2.2 & 1.4 & +1.7 & 4.4 & 3.3 & 3.9 & -1.1 \\
\hline 650 & 1.7 & 3.1 & 2.4 & +1.4 & 1.1 & 2.8 & 1.9 & +1.7 & 2.2 & 3.3 & 2.8 & +1.1 \\
\hline 750 & 2.8 & 2.5 & 2.6 & -2.8 & 3.3 & 2.2 & .2 .8 & -1.1 & 2.2 & 2.8 & 2.5 & +0.6 \\
\hline 850 & 1.9 & 3.3 & 2.6 & +1.4 & 1.1 & 2.8 & 1.9 & +1.7 & 2.8 & 3.9 & 3.3 & +1.1 \\
\hline 950 & 3.3 & 2.5 & 2.9 & -8.3 & 3.3 & 2.2 & 2.8 & -1.1 & 3.3 & 2.8 & 3.1 & -0.6 \\
\hline Sum: & +2.6 & -3.0 & 2.8 & +3.7 & 1.7 & 2.3 & 2.0 & +0.6 & 3.5 & 3.6 & 3.5 & +1.9 \\
\hline
\end{tabular}


analysis of the reaction time data yielded a significant main effect for pseudodominance (the item mean analysis approached significance), both the subject mean and item mean analysis of error rates yielded a main effect for pseudodominance $\left[F_{1}(1,153)=13.125\right.$, $\left.\mathrm{p}<.001 ; \mathrm{F}_{2}(1,38)=10.259, \mathrm{p}<.004\right]$. This analysis indicates that the $3.5 \%$ errors that occurred in response to "LCD" nonword targets was significantly greater than the $2.0 \%$ errors that occurred in response to "HCD" nonword targets. Therefore the error data provide even stronger support for the patterns suggested by the reaction time data analysis.

In summary, three observations may be stated about the nonword target data. First, subjects were slower to respond to nonword-targets than to word-targets. Second, subjects were generally faster and more accurate in response to nonword targets derived from HCD words than to nonword targets derived from LCD words. As shown in Table 11 and Figure 4, this trend was observed in all but the 950 SOA condition. Finally, there was a trend for subjects to respond faster to nonwords preceded by word primes than to nonwords preceded by neutral primes, but only when the nonwords were derived from HCD words. However, this nonword facilitation effect was not statistically reliable.

\section{Discussion}

\section{Word Target Data}

In general, these results support three findings that have been reported in the literature. First, responses to word targets were faster than responses to nonword targets. This is to be expected if one assumes that an exhaustive search of the lexicon is 


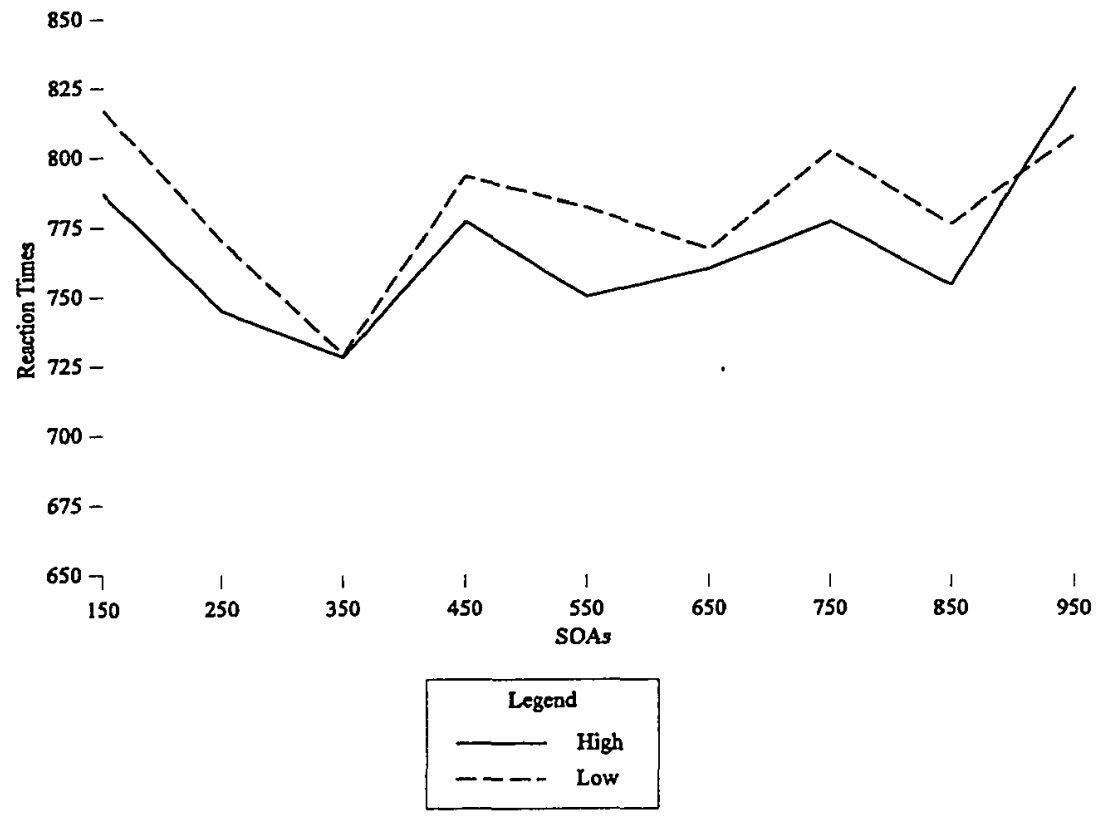

Figure 4. Mean reaction time (in milliseconds) for correct responses to "HCD" and "LCD" nonword targets as a function of SOA. 
required before a nonword target can be rejected (Forster, 1976). Second, responses to word targets preceded by a related prime were faster and more accurate than responses to word targets preceded by an unrelated prime. This observation confirms the standard semantic priming effect first reported by Meyer and Schvaneveldt (1971). Finally, responses to $H C D$ targets were faster and more accurate than responses to LCD targets. This result was especially robust, and was observed both within and across all SOA and priming conditions, without exception. Recall that in this experiment, the high-versus low-category dominance variable was confounded with word frequency (the mean frequency of occurrence for critical HCD word targets was 81.40 , and for critical LCD word targets was 20.83). Therefore, this finding likely replicates the standard word frequency effect, which is the well established finding that responses are faster and more accurate to high-frequency words than to low-frequency words (Rubenstein, Garfield, \& Millikan, 1970; Whaley, 1978). The degree to which dominance alone contributed to this effect, or whether a pure "dominance effect," exists, independent of word frequency, is not clear. Den Heyer, Briand, Smith (1985) reported a dominance effect, even though they controlled for frequency so that targets of dissimilar dominance were roughly equivalent in terms of word frequency. However, Becker (1980), who also controlled for frequency across three levels of category dominance, did not find a dominance effect. Lorch, Balota, and Stamm (1986) also reported a dominance effect, but did not report whether the two levels of dominance they used were matched for word frequency. 
The prediction that a pattern of facilitation without inhibition would be observed in short SOA conditions followed by a pattern of either facilitation and inhibition, or inhibition without facilitation, in the longer SOA conditions was only partially met. As expected, facilitation without inhibition was observed in the shortest SOA conditions (see Table 6). However, the only SOA condition in which a significant amount of inhibition was observed was the 450 SOA condition. If inhibitory effects are the hallmark of time-consuming strategic processing, it is puzzling that significant inhibitory effects were observed in only the 450 SOA condition, and not in the longer SOA conditions. Despite the failure to observe significant inhibitory effects in the longest SOA conditions, the patterns of facilitation and inhibition observed in the 150 $\mathrm{ms}, 250 \mathrm{~ms}, 350 \mathrm{~ms}$, and $450 \mathrm{~ms}$ SOA conditions are consistent with the claim that only automatic spreading activation contributes to priming in short SOA conditions, whereas strategic processing is observed with longer SOAs. These results will be discussed in greater detail in the general discussion.

\section{Nonword Target Data}

The results obtained in the nonword target condition are perplexing for two reasons. First, subjects tended to be faster and more accurate in their response to nonword targets derived from HCD words than in response to nonword targets derived from LCD words. This result was unexpected and suggests that subjects were detecting a subtle difference between these two groups of nonword targets. Second, their was an insignificant trend for subjects to respond faster to nonwords preceded by word primes than to nonwords preceded by neutral primes, especially when the nonwords were 
derived from HCD words. It is important to recall that in all cases, the word prime that preceded a nonword target was unrelated to the word from which the nonword target was derived. Therefore, this observation cannot be explained in terms of the subjects' somehow detecting a relation between the word prime and the nonword target "source," because in its undistorted form, the nonword was never related to the prime.

The prediction that a nonword facilitation effect would be observed only in the longer SOA conditions was clearly not met. In fact, this effect failed to reach statistical significance in any condition. This result contrasts with the results of several other investigators who have reported a nonword facilitation effect, especially in long SOA conditions. There was an inconsistent trend for larger nonword facilitation effects in the longer SOA conditions for "HCD" but not "LCD" targets (see Table 11). However, the presence of a "pseudodominance effect" makes the nonword data difficult to interpret. Since it is not clear how to best interpret this effect, and since the main effect for priming did not reach statistical significance, the nonword data were not especially informative.

\section{End Notes}

1. DMASTR software was developed by Kenneth I. Forster and Jonothan C. Forster at Monash University and at the University of Arizona. Detailed information regarding this software is available at the following website: www.dundee.ac.uk.psychology/ dmastr/ dmastr.htm

2. Tukey HSD cv refers to the Tukey highly significant difference critical value which is computed as the standard error times the $q$ value. If the difference between any of the means exceeds the critical value, the difference is signficant at the specified $p$ value. 


\section{EXPERIMENT 2}

\section{Pilot Study 1}

The purpose of Experiment 2 was to determine whether Broca's aphasics would display the same pattern of facilitatory and inhibitory effects across SOAs as do normal subjects, in a pairwise visual lexical decision task using a high relatedness proportion and a high nonword ratio. However, the availability of appropriate research subjects precluded a direct replication of Experiment 1. When investigating language processing in aphasia, the availability of appropriate research subjects always poses a challenge. Typically it is necessary to use a within-subjects design because only small numbers of subjects who meet appropriate selection criteria can be located.

To examine the impact of SOA within subjects, it is necessary to expose each subject to the same stimuli at various SOA conditions. This introduces the potential confound of repetition effects. Although a number of investigators have demonstrated that repetition effects and semantic priming effects are additive (Den Heyer, Goring, \& Dannenbring, 1985; Durgunoglu, 1988, Wilding, 1986), word frequency effects do interact with repetition effects (e. g., Scarborough, Cortese, \& Scarborough, 1977). That is, whereas overall semantic priming effects are unaffected by repeated stimulus presentations, low frequency words benefit more (i e., are responded to faster) from repetition than do high frequency words. This posed a potential problem for Experiment 2, since the high-category dominance exemplar targets are substantially higher in word frequency than are the low-category dominance exemplar targets. 
Therefore, a pilot study was run simultaneous to Experiment 1 to determine if similar results could be obtained with repeated presentation of the same stimuli within subjects.

\section{Methods}

\section{Design}

The same design used for Experiment 1 was used for this pilot study, with two exceptions. First, the SOA condition was treated as a within-groups factor. Second, to limit the number of subjects needed, only 6 SOA conditions were examined. These included the $250,350,450,550,650$, and 750 SOA conditions. Thus the design for the analysis of word targets in the pilot study was a 6 (SOA) $\times 3$ (prime condition) $\mathrm{x} 2$ (dominance condition) design, with all conditions being treated as within subjects factors.

\section{Subjects}

Six undergraduate students from the Louisiana State University served as subjects. The same subject selection criteria used for Experiment 1 were used for the pilot study. Each of these six subjects performed the lexical decision task six times during six separate experimental sessions. A different version of the six stimulus lists (A-F, as described for Experiment 1) were presented in each of the six experimental sessions. The six SOA conditions were counterbalanced across subjects and list versions as described below. Subjects received extra course credit for their participation. One subject was replaced, and her data discarded, because she failed to return for the sixth experimental session. 


\section{Stimuli and Procedure}

The same stimulus lists that were used in Experiment 1 were used for Pilot 1. Recall that six versions of the base stimulus list were formed to counterbalance critical target items across priming conditions. In order to create the most distance between presentations of identical prime target pairs (and therefore, hopefully reduce the impact of repetition effects), the stimulus lists were presented to each subject in the following order: ADEBCF. This ordering is depicted in Table 13. Ordering the individual lists in this manner assured that identical prime-NW target pairs were alternated across every other experimental session, and that identical prime-word target pairs were separated by two experimental sessions. SOA conditions were counterbalanced across subjects, and across list version. Table 14 depicts how this was accomplished. Table 14 also includes examples of prime-target pairs for each priming condition, to illustrate how these are paired across the six list versions.

The purpose of counterbalancing and ordering the stimulus presentations in this way was to assure that any effects of repetition would be distributed equally across SOA, priming, and category dominance conditions. ${ }^{1}$ Counterbalancing SOA conditions across subjects and lists, and presenting the list versions in the manner described above assured that 1 ) the number of repetitions per each priming condition ( $R, N, U$ for words, and $\mathrm{W}, \mathrm{N}$ for nonwords) was equal, 2) each priming condition appeared an equal number of times in each presentation order and with each SOA condition, 3) the first presentation of each identical prime-target pair was maximally separated from the second presentation of the same pair, 4) the first and second presentation of each 
Table 13. Proposed stimulus list sequence for pilot study.

\begin{tabular}{|c|c|c|c|c|c|c|}
\hline List & \multicolumn{2}{|c|}{ Word Target Conditions } & \multicolumn{4}{|c|}{ NW Target Conditions } \\
\hline A & cloth & COTTON & body & PAMB & blank & GOCK \\
\hline $\mathrm{D}$ & blank & COTTON & blank & PAMB & body & GOCK \\
\hline $\mathrm{E}$ & fruit & COTTON & body & PAMB & blank & GOCK \\
\hline $\mathrm{B}$ & cloth & COTTON & blank & PAMB & body & GOCK \\
\hline $\mathrm{C}$ & blank & COTTON & body & PAMB & blank & GOCK \\
\hline $\mathrm{F}$ & fruit & COTTON & blank & PAMB & body & GOCK \\
\hline
\end{tabular}


Table 14. Related conditions in bold that were analyzed together for a single SOA condition.

\begin{tabular}{|c|c|c|c|c|c|c|}
\hline Session: & 1st & 2nd & $3 \mathrm{rd}$ & 4th & 5 th & 6 th \\
\hline List : & A & $\mathrm{D}$ & $\mathrm{E}$ & B & $\mathrm{C}$ & $\mathrm{F}$ \\
\hline \multirow[t]{9}{*}{ Wd Pairs: } & cloth-COTTON-R & blank-COTTON-N & fruit-COTTON-U & cloth-COTTON-R & blank-COTTON-N & fruit-COTTON-U \\
\hline & animal-DOG- $R$ & blank-DOG-N & vehicle-DOG-U & animal-DOG-R & blank-DOG-N & vehicle-DOG-U \\
\hline & color-BLUE-R & blank-BLUE-N & vegetable-BLUE-U & color-BLUE-R & blank-BLUE-N & vegetable-BLUE-U \\
\hline & blank-RAYON-N & tree-RAYON-U & cloth-RAYON-R & blank-RAYON -N & tree-RAYON-U & cloth-RAYON-R \\
\hline & blank-HORSE-N & clothing-HORSE-U & animal-HORSE-R & blank-HORSE-N & clothing-HORSE-U & animal-HORSE-R \\
\hline & blank-RED-N & cloth-RED-U & color-RED-R & blank-RED-N & cloth-RED-U & color-RED-R \\
\hline & flower-WOOL-U & cloth -WOOL-R & blank-WOOL-N & flower-WOOL--U & cloth -WOOL-R & blank-WOOL-N \\
\hline & weapon-CAT-U & animal-CAT-R & blank-CAT-N & weapon-CAT-U & animal-CAT-R & blank-CAT-N \\
\hline & crime-GREEN-U & color-GREEN-R & blank-GREEN-N. & crime-GREEN-U & color-GREEN-R & blank-GREEN-N \\
\hline \multirow[t]{2}{*}{ NW Pairs: } & body-PAMB-W & blank-PAMB-N & body-PAMB-W & blank-PAMB-N & body-PAMB-W & blank-PAMB-N \\
\hline & blank-GOCK-N & body-GOCK-W & blank-GOCK-N & body-GOCK-W & blank-GOCK-N & body-GOCK-W \\
\hline Subj 1 & 250 & 350 & 450 & 550 & 650 & 750 \\
\hline Subj 2 & 350 & 450 & 550 & 650 & 750 & 250 \\
\hline Subj 3 & 450 & 550 & 650 & 750 & 250 & 350 \\
\hline Subj 4 & 550 & 650 & 750 & 250 & 350 & 450 \\
\hline Subj 5 & 650 & 750 & 250 & 350 & 450 & 550 \\
\hline Subj 6 & 750 & 250 & 350 & 450 & 550 & 650 \\
\hline
\end{tabular}


identical prime target pair was evenly distributed across SOAs (e.g. cloth-COTTON was presented with a $250 \mathrm{~ms}$ SOA on both its first and a second occurrence, with a 350 ms SOA on both its first and a second occurrence, and so on, and 5) identical prime target pairs that were presented first with a short SOA were presented to the same subject with a longer SOA in the second presentation, and half that were first presented with a longer SOA were presented to the same subject with a shorter SOA on the second presentation (for example, subject-1 first saw the pair 'cloth-COTTON' with a $250 \mathrm{SOA}$, and subsequently with a 550 SOA, subject-4 first saw 'cloth-COTTON' with a 450 SOA, and later with a $150 \mathrm{SOA})$.

Table 14 illustrates with bolded text how related conditions collected across subjects and experimental sessions were analyzed together for a single SOA condition. Within each list version there were 24 (12 high- and 12 low-dominance) critical observations for each word-target priming condition (related, unrelated, and neutral) and 24 critical observations for each nonword-target priming condition (word-NW and neutral-NW). This yielded 144 measurements for each priming condition for each SOA condition, and 72 measurements for each dominance condition for each SOA condition. In order to further reduce the potential impact of repetition effects, experimental sessions were spaced two to three days apart by placing subjects on a Monday, Wednesday, Friday schedule for two consecutive weeks. Scarborough, Cortese, and Scarborough (1977) and Den Heyer, Goring, and Dannenbring (1985) reported data that suggest that spacing experimental sessions in this way would help reduce the confound of repetition effect. 


\section{Results}

The same critical target items that were removed from the Experiment 1 data analysis were removed from the Pilot 1 data analysis (and all subsequent pilot data analyses). Therefore, reaction times and error responses to a total of 20 critical target items (10 HCD and $10 \mathrm{LCD})$ were analyzed for each word-target priming condition (related, unrelated, and neutral). Nonword target data were not considered for analysis because of their limited informativeness in the Experiment 1 data. Recall that for Pilot 1, only six SOA conditions were used, $250,350,450,550,650$, and $750 \mathrm{~ms}$. Also, since each subject completed the experiment 6 times, once each with each SOA condition, SOA served as a within subjects factor.

The mean reaction times (RTs) for correct responses to word targets in each of the three priming conditions (related, unrelated, and neutral), the two dominance conditions (HCD and LCD), and each SOA condition, are presented in Table 15. These mean values were calculated after missing values and extreme values were replaced with each subject's mean RT per condition. No individual RTs exceeded $1999 \mathrm{~ms}$. Missing value replacements accounted for $3.38 \%$ of the data and extreme value replacements accounted for $4.12 \%$ of the data. Differences between the means displayed in Table 15 are reported in Table 16 as facilitation (neutral minus related), inhibitory (neutral minus unrelated), and overall priming (unrelated minus related) effects. Note that some degree of overall priming was observed in all but three conditions (for HCD targets in the 350 and 450 SOA conditions, and for LCD targets in 
Table 15. Pilot 1 reaction time data summary. Mean reaction times in milliseconds to word-target pairs in all dominance, priming ( $R=$ related, $U=$ unrelated, $N=$ neutral, and SOA conditions.

\begin{tabular}{|c|c|c|c|c|c|c|c|c|c|c|c|c|c|c|}
\hline \multirow[b]{2}{*}{ Priming Condition: } & \multicolumn{3}{|c|}{$\begin{array}{l}\text { High and Low } \\
\text { Dominance }\end{array}$} & \multirow[b]{2}{*}{ Mean } & . & \multicolumn{3}{|c|}{$\begin{array}{c}\text { High } \\
\text { Dominance }\end{array}$} & \multirow[b]{2}{*}{ Mean } & . & \multicolumn{3}{|c|}{$\begin{array}{c}\text { Low } \\
\text { Dominance }\end{array}$} & \multirow[b]{2}{*}{ Mean } \\
\hline & $\underline{\mathrm{R}}$ & $\underline{\mathrm{U}}$ & $\underline{N}$ & & . & $\underline{\mathrm{R}}$ & $\underline{U}$ & $\mathrm{~N}$ & & & $\underline{\mathrm{R}}$ & $\underline{\mathrm{U}}$ & $\underline{\mathbf{N}}$ & \\
\hline$\underline{\mathrm{SOA}}$ & & & & & • & & & & & & & & & \\
\hline 250 & 570 & 578 & 567 & 572 & . & 517 & 550 & 539 & 535 & & 623 & 606 & 596 & 609 \\
\hline 350 & 566 & 578 & 576 & 574 & . & 541 & 542 & 543 & 542 & & 591 & 615 & 610 & 574 \\
\hline 450 & 620 & 637 & 625 & 627 & . & 595 & 589 & 585 & 590 & & 644 & 685 & 664 & 627 \\
\hline 550 & 595 & 615 & 610 & 607 & . & 564 & 591 & 572 & 576 & & 626 & 639 & 649 & 607 \\
\hline 650 & 557 & 591 & 606 & 585 & . & 532 & 564 & 589 & 562 & & 583 & 619 & 623 & 585 \\
\hline 750 & 609 & 628 & 629 & 621 & . & 586 & .606 & 613 & 602 & & 632 & 650 & 644 & 622 \\
\hline Mean: & 586 & 605 & 602 & $\underline{598}$ & . & 556 & 574 & 574 & $\underline{568}$ & & 617 & 636 & 631 & $\underline{628}$ \\
\hline
\end{tabular}


Table 16. Pilot 1 priming effect summary. Priming effects for each SOA and dominance condition representing the differences between mean reaction times for each of the three priming conditions: Facil (neutral minus related), Inhib (neutral minus unrelated) and Overall (unrelated minus related).

\begin{tabular}{|c|c|c|c|c|c|c|c|c|c|c|c|}
\hline \multirow[b]{2}{*}{ Priming Effects: } & \multicolumn{3}{|c|}{$\begin{array}{l}\text { High and Low } \\
\text { Dominance }\end{array}$} & \multirow{2}{*}{$\begin{array}{l}\cdot \\
\dot{v} \\
\cdot\end{array}$} & \multicolumn{3}{|c|}{$\begin{array}{c}\text { High } \\
\text { Dominance }\end{array}$} & \multicolumn{4}{|c|}{$\begin{array}{c}\text { Low } \\
\text { Dominance }\end{array}$} \\
\hline & $\underline{\text { Facil }}$ & $\underline{\text { Inhib }}$ & Overall & & $\underline{\text { Facil }}$ & $\underline{\text { Inhib }}$ & Overall & . & $\underline{\text { Facil }}$ & $\underline{\text { Inhib }}$ & Overall \\
\hline$\underline{\mathrm{SOA}}$ & & & & . & & & & . & & & \\
\hline 250 & -03 & -11 & +07 & . & +21 & -11 & +32 & . & -27 & -11 & -17 \\
\hline 350 & +10 & -02 & +12 & . & +01 & +01 & 0 & . & +18 & -05 & +24 \\
\hline 450 & +05 & -12 & +17 & . & -09 & -04 & -05 & . & +19 & -21 & +40 \\
\hline 550 & +15 & -05 & +20 & . & +08 & -20 & +28 & . & +23 & +10 & +13 \\
\hline 650 & $+49^{a}$ & +15 & $+34^{h}$ & . & $+57^{\mathrm{h}}$ & +25 & +32 & . & +40 & +04 & +36 \\
\hline 750 & +20 & +01 & +19 & . & +28 & +07 & +20 & . & +12 & -06 & +18 \\
\hline Mean: & +16 & -02 & $+18^{-}$ & . & +18 & 0 & $+18^{-}$ & . & +14 & -05 & +19 \\
\hline
\end{tabular}

Note: Priming effects without superscript are not significant by either analysis. ${ }^{2}$ Significant at the .01 level by both the subject and item analysis. 'Significant at the .05 level by both the subject and item analysis. 'Significant at the .01 level by the subject analysis and at the .05 level by the item analysis. 'Significant at the .05 level by the subject analysis and at the .01 level by the item analysis. ' Significant at the .05 level by the subject analysis but not significant by the item analysis. 'Significant at the .01 level by the subject

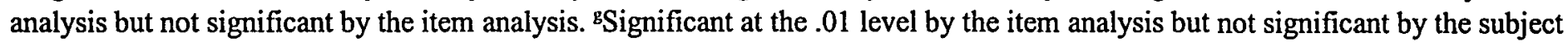
analysis. "'Significant at the .05 level by the item analysis but not significant by the subject analysis. -Main effect for priming was significant, but individual differences between means did not reach significant level with Tukey post hoc analysis. 
the 250 SOA condition), although in all conditions the magnitude of the effect is considerably smaller than that observed in Experiment 1 (see Table 6).

A separate 6(SOA) $\times 3$ (prime) $\times 2$ (dominance) ANOVA was performed with subject means (collapsed across targets) and item means (collapsed across subjects). For the subject mean analysis, all three factors were treated as within subjects factors. For the item mean analysis dominance served as a between items factor and SOA and prime served as within items factors.

The only main effect that was significant with the subject means analysis was a main effect for dominance, which was also significant with the item means analysis $\left[F_{1}\right.$ $\left.(1,5)=34.250, \mathrm{p}<.003 ; \mathrm{F}_{2}(1,58)=59.235, \mathrm{p}<.001\right]$. This analysis indicates that, averaged across SOA and prime conditions, the $60 \mathrm{~ms}$ difference between the $568 \mathrm{~ms}$ mean RT to HCD targets and the 628 ms mean RT to LCD targets was statistically reliable. These means, and the mean RTs for HCD targets and LCD targets in each SOA condition, are shown in the last two panels of Table 15. Note that as in Experiment I, RTs to HCD targets are faster than RTs to LCD targets in each of the individual SOA conditions.

The main effect for prime was significant with the item analysis but not the subject analysis $\left[F_{1}(2,10)=2.693, p<.12 ; F_{2}(1,116)=3.843, p<.03\right]$. As shown at the bottom of the first panel of Table 16, the mean RT to word targets (collapsed across pseudodominance and SOA conditions) was $16 \mathrm{~ms}$ faster in the related condition than in the neutral condition (facilitation), 2 ms slower in the unrelated condition than in the neutral condition (inhibition), and $18 \mathrm{~ms}$ faster in the related condition than in the 
unrelated condition (overall priming). A Tukey post hoc analysis of the differences between these means (averaged across items) indicated that none of these differences were significant [Tukey HSD $\mathrm{cv}=41.87, \mathrm{p}<.05$ ]

The main effect for SOA was also reliable only on the item mean analysis $\left[F_{1}\right.$ $\left.(5,25)=.504, p<.78 ; F_{2}(5,290)=24.438, p<.001\right]$. The mean RT for each SOA condition, averaged across pseudodominance and priming conditions, is shown in the last column of the first panel of Table 15. Tukey post hoc analyses of the differences between these means (across items) [Tukey HSD cv $=34.00, \mathrm{p}<.05, \mathrm{cv}=40.34, \mathrm{p}<$ $.01]$ indicate that the mean RTs obtained in the 450 and 750 SOA conditions were slower than those in the 250,350 , and 550 SOA conditions, and that the mean RT obtained in the 550 condition was reliably slower than that obtained in the 250 condition. No other differences were significant.

In summary, responses to $\mathrm{HCD}$ word targets were faster than responses to $\mathrm{LCD}$ word targets within each individual SOA and dominance condition. This finding replicates the dominance effect observed in Experiment 1. However, the priming effects observed in the Pilot 1 data were clearly not sufficiently robust to replicate the results of Experiment 1. As may be seen in Table 16, there was a trend for subjects to respond more quickly in the related priming condition compared to the neutral and unrelated conditions. There was also a somewhat less consistent trend for subject to respond more slowly in the unrelated priming condition compared to the neutral condition. However, a main effect for prime was only observed with the item analysis. Furthermore, post hoc analysis of the difference between mean RTs in the 
three priming conditions revealed no significant individual priming effects. Since analysis of the primary data of interest (i.e. reaction time means) obtained in Pilot 1 failed to replicate the results of Experiment 1, no further analyses of the Pilot 1 data were conducted.

\section{Discussion}

The pilot data clearly replicated the dominance effect observed in Experiment 1, although the magnitude of the effect was smaller. However, despite the careful counterbalancing and spacing of experimental sessions, the results of Pilot 1 failed to replicate the priming results observed in Experiment 1. An obvious factor that may have contributed to this finding is that individual differences between subjects may have been too great. Of course this is always a problem when conducting research with a small number of subjects. A clear example of how damaging this problem could potentially be was identified in the Experiment 1 data by examining individual subject's priming effects. Within each $\mathrm{SOA} /$ dominance condition, an average of 5 subjects (out of the total 18 subjects per SOA group) displayed an pattern of priming that was opposite that of the group. For example, in the 450 SOA condition, mean reaction times across the entire group showed a clear pattern of inhibition dominance. Within each dominance condition however, the mean reaction times of five subjects reflected a pattern of facilitation dominance. Had these subjects been included in a study with a smaller $\mathrm{n}$, the statistical analysis would have yielded quite different results. Small $\mathrm{n}$ studies always run the risk of including a higher proportion of subjects whose performance does not conform to the norms identified in larger $\mathbf{n}$ studies. Such may 
have been the case with the subjects who were included in the pilot study. Nonetheless, this is a problem that is difficult to circumvent when conducting research with special populations that limit subject availability.

The fact that the each subject was exposed to the same task six times in the present experiment may also have contributed to the failure to replicate the priming results of Experiment 1. As noted earlier, den Heyer, Goring \& McPherson (1985) reported that repetition effects and semantic priming effects are additive, as are practice effects and semantic priming effects. However, in their work, stimuli were presented with the same SOA, across 3 sessions. In the present data, it is not possible to determine how repetition and practice effects may have interacted with variations in SOA across a total of six sessions. One cannot discount the possiblity that these factors also contributed to the rather weak effects that were observed, despite the steps taken to minimize their influence.

Bushell (1996) replicated the standard relatedness proportion effect by varying the relatedness proportion across two experimental sessions with a total of eight subjects. Her work demonstrated that it is possible to replicate a standard semantic priming effect using a within subjects design with a small number of subjects. This suggests that one way to increase the likelihood of replicating the results of Experiment 1 might be to reduce the number of sessions/SOA conditions. In order to address this possibility, two pilot studies were planned. The first of these was designed to determine if the results obtained in the $250 \mathrm{~ms}$ and $450 \mathrm{~ms}$ SOA conditions in Experiment 1 could be replicated with a smaller group of subjects, using the same between subjects design 
that was used in Experiment 1. The second of these was designed to determine if these results could be replicated by manipulating SOA within-subjects. The $250 \mathrm{SOA}$ condition was selected since the results obtained in this condition in Experiment 1 clearly conformed to a pattern consistent with automatic spreading activation. Similarly, the 450 SOA condition was selected since the results obtained in that condition conformed to a pattern consistent with strategic processing. Thus, examining priming in only these two SOA conditions continued to provide a means for examining the nature of automatic versus strategic priming effects.

Pilot Study 2. A.

\section{Methods}

Procedure

The purpose of Pilot Study 2. A. was to determine if the results obtained in the $250 \mathrm{~ms}$ and $450 \mathrm{~ms}$ SOA conditions could be replicated with a smaller group of subjects, using the same between subjects design used in Experiment 1. The stimulus materials and procedure were identical to those used in Experiment 1 with one exception. All subject's whose reaction time standard deviation exceeded $200 \mathrm{~ms}$ were replaced without further consideration of their data. This procedure was implemented to further reduce variability in the data

\section{Subjects}

Twelve undergraduate students from the Louisiana State University served as subjects. Selection criteria were identical to those used for Experiment 1 . Six subjects participated in each of the two SOA conditions. All subjects received extra credit for 
participation in the study. All subjects whose overall error rate exceeded $10 \%$, or whose reaction time standard deviation exceed $200 \mathrm{~ms}$, were replaced, and their data were discarded. A total of 5 subjects were replaced due to high standard deviations, and a total of 3 subjects were replaced due to error rates in excess of $10 \%{ }^{2}$

\section{$\underline{\text { Results }}$}

Reaction Time Analysis.

The mean reaction times (RTs) for correct responses to word targets in each of the three priming conditions (related, unrelated, and neutral), the two dominance conditions (HCD and LCD), and each SOA condition, are presented in the upper half of Table 17. Differences between these means are reported in the lower half of the table. Before these mean values were calculated, all RTs $\geq 2000 \mathrm{~ms}$ were converted to errors. These long latencies occurred on $.97 \%$ of the correct word target responses. To further reduce variability in the data, each subject's data was cleaned individually. Missing values (errors) and extreme values (RTs +/- 2 SD from the subject's mean per condition) were replaced with the subject's mean RT for that condition. Missing value replacements accounted for $2.5 \%$ of the data and extreme value replacements accounted for $3.47 \%$ of the data.

A separate 2(SOA) $\times$ 3(prime) $\times 2$ (dominance) ANOVA was performed with subject means (averaged across items) and item means (averaged across subjects). For the subject mean analysis, SOA was treated as a between subjects factor, and prime and dominance served as within subjects factors. For the item mean analysis dominance served as a between items factor and SOA and prime served as within items factors. 
Table 17. Pilot 2.A. word-target data summary. Mean reaction times in milliseconds to word-target pairs in all dominance, priming ( $R=$-related, $U=$ =unrelated, $N=$ neutral, and SOA conditions; and priming effects for each SOA and dominance condition representing the differences between mean reaction times for each of the three priming conditions. Facil (neutral minus related), Inhib (neutral minus unrelated) and Overall (unrelated minus related).

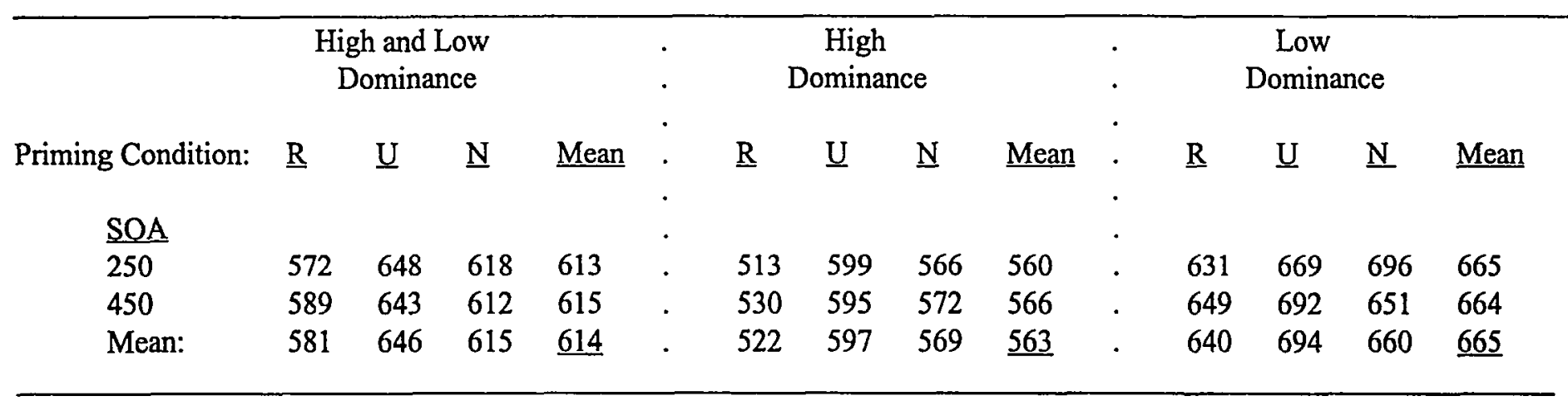

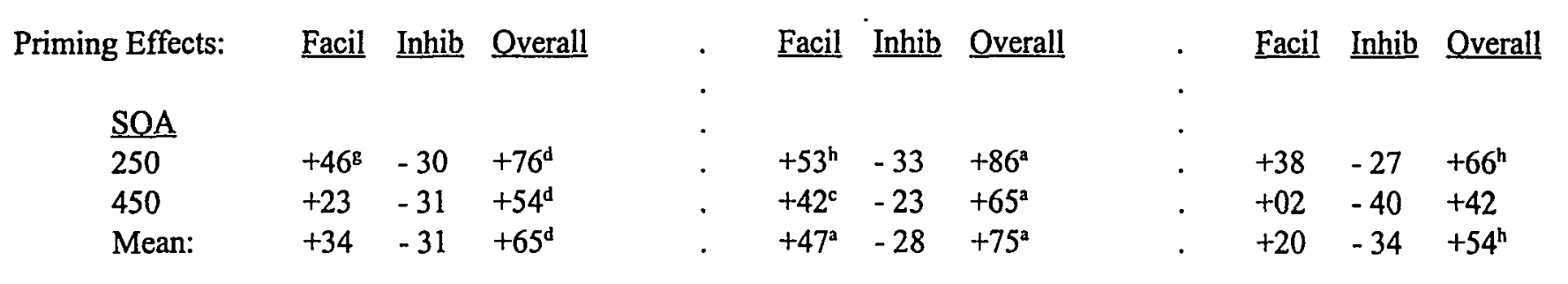

Note: Priming effects without superscript are not significant by either analysis. ${ }^{2}$ Significant at the .01 level by both the subject and item analysis. "Significant at the .05 level by both the subject and item analysis. 'Significant at the .01 level by the subject analysis and at the .05 level by the item analysis. 'Significant at the .05 level by the subject analysis and at the .01 level by the item analysis. ' Significant at the .05 level by the subject analysis but not significant by the item analysis. 'Significant at the .01 level by the subject analysis but not significant by the item analysis. ${ }^{8}$ Significant at the .01 level by the item analysis but not significant by the subject analysis. 'Significant at the .05 level by the item analysis but not significant by the subject analysis. - Main effect for priming was significant, but individual differences between means did not reach significant level with Tukey post hoc analysis. 
The main effect for prime was reliable on both analyses $\left[F_{1}(2,20)=9.09, p<\right.$ $\left..002 ; F_{2}(2,116)=20.56, p<.001\right]$. The difference between the mean RTs for each of the three priming conditions (averaged across SOA and dominance conditions) was examined using a Tukey post hoc analysis. These mean values are shown in the top half of Table 17. The difference values are shown on the last line of the first panel of the table. The $+65 \mathrm{~ms}$ overall priming effect was significant with both the subject mean $[$ Tukey HSD cv $=54.56, \mathrm{p}<.05]$ and item mean [Tukey HSD cv $=42.50, \mathrm{p}<.01]$ analysis. The $+34 \mathrm{~ms}$ facilitation effect approached statistical significance only with the item mean analysis [Tukey HSD cv $=34.00, \mathrm{p}<.05$ ]. The $-31 \mathrm{~ms}$ inhibition effect was not statistically significant by either analysis.

The main effect for dominance was also reliable on both analyses $\left[F_{1}(1,10)=\right.$ $\left.39.09, \mathrm{p}<.001 ; \mathrm{F}_{2}(1,58)=75.11, \mathrm{p}<.001\right]$. This analysis indicates that, collapsed across SOA and prime conditions, the $102 \mathrm{~ms}$ difference in mean RT to HCD targets (563 ms) and the mean RT to LCD targets ( $665 \mathrm{~ms}$ ) was statistically reliable. No other main effects or interactions were significant.

In order to determine whether the facilitation, inhibition, and overall priming effects were significant in each of the individual SOA/dominance conditions, a separate one-way ANOVA for subject and item mean RTs obtained in the three priming conditions was performed separately for each of the individual SOA/dominance conditions. These means are reported in the upper half of the last two panels of Table 17 , and the differences between the means are reported in the lower half of the table. For those conditions in which a main effect of priming was observed, Tukey post hoc 
analyses were performed to determine the significance levels of the differences between the means. Relevant statistics from these analyses are reported in Table 18. As shown in Table 18, priming was significant for $\mathrm{HCD}$ targets in the 250 and the $450 \mathrm{SOA}$ conditions, by both the subject and item mean analysis. For LCD targets, the only analysis that yielded a significant effect was the item analysis of means obtained in the 250 condition. Significance levels for the individual priming effects, as determined by Tukey post hoc analyses, are indicated by superscript in Table 17. In the 250 SOA condition for $\mathrm{HCD}$ targets, the $+86 \mathrm{~ms}$ overall priming effect was significant by both subject and item mean analyses, and the $+53 \mathrm{~ms}$ facilitation effect was significant by the item analysis. In the $450 \mathrm{SOA}$ condition for $\mathrm{HCD}$ targets, the $+65 \mathrm{~ms}$ overall priming effect and the $+42 \mathrm{~ms}$ facilitation effect were significant by both analysis. The $-23 \mathrm{~ms}$ inhibition effect was not significant. In the 250 SOA condition for LCD targets, the +66 ms overall priming effect was reliable by the item, but not the subject analysis. Neither the $+38 \mathrm{~ms}$ facilitation effect, nor the $-27 \mathrm{~ms}$ inhibition effect, were significant.

\section{Error Analysis}

More errors occurred in response to LCD word targets (16) than in response to HCD word targets (2). Also, the greatest number of errors occurred in the unrelated condition (11), and more errors were made in the neutral condition (5) than in the related condition (2). Since errors only account for $2.5 \%$ of the word target data, and, in general, support the reaction time data, they were not analyzed further. 
Table 18. Results of one-way ANOVAs for mean RTs obtained in the the three priming conditions for each SOA/dominance condition in Pilot 1.

\begin{tabular}{|c|c|c|c|c|c|c|c|c|}
\hline \multicolumn{9}{|c|}{ HCD and LCD Targets } \\
\hline \multirow{3}{*}{$\begin{array}{l}\text { SQA } \\
250\end{array}$} & \multirow{2}{*}{\multicolumn{2}{|c|}{$E_{1}(2,10), p$ value }} & \multicolumn{2}{|c|}{$\begin{array}{l}\text { Tukey CV } \\
\mathrm{q}(3,10)\end{array}$} & & & \multicolumn{2}{|c|}{$\begin{array}{r}\text { Tukey CV } \\
\text { q }(3,118)\end{array}$} \\
\hline & & & .05 & .01 & \multicolumn{2}{|c|}{$\mathrm{E}_{2}(2,118), \mathrm{p}$ value } & .05 & .01 \\
\hline & .18 & $<.84$ & $\mathrm{n} / \mathrm{a}$ & $\mathrm{n} / \mathrm{a}$ & .33 & $<.72$ & $\mathrm{n} / \mathrm{a}$ & $\mathrm{n} / \mathrm{a}$ \\
\hline 350 & .48 & $<.63$ & $\mathrm{n} / \mathrm{a}$ & $\mathrm{n} / \mathrm{a}$ & .23 & $<.80$ & $\mathrm{n} / \mathrm{a}$ & $\mathrm{n} / \mathrm{a}$ \\
\hline 450 & .70 & $<.52$ & $n / a$ & $\mathrm{n} / \mathrm{a}$ & .51 & $<.61$ & $\mathrm{n} / \mathrm{a}$ & $\mathrm{n} / \mathrm{a}$ \\
\hline 550 & .50 & $<.62$ & $\mathbf{n} / \mathbf{a}$ & $\mathbf{n} / \mathbf{a}$ & .83 & $<.44$ & $\mathrm{n} / \mathrm{a}$ & $\mathrm{n} / \mathrm{a}$ \\
\hline 650 & 4.09 & $<.05$ & 47.30 & 64.24 & 5.96 & $<.01$ & 34.30 & 42.88 \\
\hline 750 & 2.31 & $<.15$ & $\mathrm{n} / \mathrm{a}$ & $\mathrm{n} / \mathrm{a}$ & 9.28 & $<.60$ & $\mathrm{n} / \mathrm{a}$ & $\mathrm{n} / \mathrm{a}$ \\
\hline \multicolumn{9}{|c|}{ HCD Targets } \\
\hline \multicolumn{7}{|c|}{$q(3,10)$} & \multicolumn{2}{|c|}{$\mathrm{q}(3,58)$} \\
\hline 250 & 2.49 & $<.14$ & $\mathrm{n} / \mathrm{a}$ & $\mathrm{n} / \mathrm{a}$ & 2.13 & $<.13$ & $\mathrm{n} / \mathrm{a}$ & $\mathrm{n} / \mathrm{a}$ \\
\hline 350 & .01 & $<.99$ & $\mathrm{n} / \mathrm{a}$ & $\mathrm{n} / \mathrm{a}$ & .00 & $<.99$ & $\mathrm{n} / \mathrm{a}$ & $\mathrm{n} / \mathrm{a}$ \\
\hline 450 & .38 & $<.69$ & $\mathrm{n} / \mathrm{a}$ & $\mathrm{n} / \mathrm{a}$ & .08 & $<.92$ & $\mathrm{n} / \mathrm{a}$ & $\mathrm{n} / \mathrm{a}$ \\
\hline 550 & 1.38 & $<.30$ & $\mathrm{n} / \mathrm{a}$ & $\mathrm{n} / \mathrm{a}$ & 1.06 & $<.35$ & $\mathbf{n} / \mathbf{a}$ & $\mathrm{n} / \mathrm{a}$ \\
\hline 650 & 3.79 & $<.06$ & $\mathrm{n} / \mathrm{a}$ & $n / a$ & 4.12 & $<.02$ & 48.01 & $\mathrm{n} / \mathrm{a}$ \\
\hline 750 & 3.44 & $<.07$ & $\mathrm{n} / \mathrm{a}$ & $\mathrm{n} / \mathrm{a}$ & .36 & $<.71$ & $\mathrm{n} / \mathrm{a}$ & $\mathrm{n} / \mathrm{a}$ \\
\hline \multicolumn{9}{|c|}{ LCD Targets } \\
\hline \multicolumn{7}{|c|}{$q(3,10)$} & \multicolumn{2}{|c|}{$q(3,58)$} \\
\hline 250 & .39 & $<.69$ & $\mathrm{n} / \mathrm{a}$ & $\mathrm{n} / \mathrm{a}$ & .82 & $<.45$ & $\mathrm{n} / \mathrm{a}$ & $\mathrm{n} / \mathrm{a}$ \\
\hline 350 & .79 & $<.48$ & $\mathrm{n} / \mathrm{a}$ & $\mathrm{n} / \mathrm{a}$ & .44 & $<.64$ & $\mathrm{n} / \mathrm{a}$ & $\mathrm{n} / \mathrm{a}$ \\
\hline 450 & 1.12 & $<.36$ & $\mathrm{n} / \mathrm{a}$ & $\mathrm{n} / \mathrm{a}$ & 1.12 & $<.33$ & $\mathrm{n} / \mathrm{a}$ & $\mathrm{n} / \mathrm{a}$ \\
\hline 550 & .29 & $<.81$ & $\mathrm{n} / \mathrm{a}$ & $\mathrm{n} / \mathrm{a}$ & .38 & $<.69$ & $\mathrm{n} / \mathrm{a}$ & $\mathrm{n} / \mathrm{a}$ \\
\hline 650 & 2.57 & $<.13$ & $\mathrm{n} / \mathrm{a}$ & $n / a$ & 2.17 & $<12$ & $\mathrm{n} / \mathrm{a}$ & $\mathrm{n} / \mathrm{a}$ \\
\hline 750 & .63 & $<.55$ & $\mathrm{n} / \mathrm{a}$ & $\mathrm{n} / \mathrm{a}$ & .20 & $<.82$ & $\mathrm{n} / \mathrm{a}$ & $\mathrm{n} / \mathrm{a}$ \\
\hline
\end{tabular}

Reproduced with permission of the copyright owner. Further reproduction prohibited without permission. 


\section{Discussion}

The priming effects observed in the $250 \mathrm{~ms}$ and $450 \mathrm{~ms} \mathrm{SOA}$ conditions in Experiment 1 and Pilot 2.A are presented together in Table 19 for comparison purposes. Given the much smaller number of subjects used in Pilot 2.A it is no surprise that the statistical reliability of the obtained results was much less robust in the Pilot 2.A data. Nonetheless, the pattern of results are largely consistent with those observed in Experiment 1. In both Experiment 1 and Pilot 2.A, a pattern of facilitation dominance (i.e., facilitation > inhibition) was greater was observed in the 250 SOA condition, for both HCD and LCD word targets, although the facilitation effect for LCD targets in Pilot 2.A was not statistically reliable. Inhibitory effects were not significant in the 250 condition in either experiment, although there is a suggestion of greater inhibition in the Pilot 2.A data. In Experiment 1, a pattern of the inhibition dominance (i.e. inhibition > facilitation) was observed in the 450 SOA condition for both HCD and LCD word targets, and in both cases the inhibition effect was statistically reliable. In the Pilot 2.A, a pattern of inhibition dominance was observed for LCD word targets, however the inhibition effect was not statistically reliable.

Three primary differences may be noted between the results obtained in Experiment 1 and those obtained in Pilot 2.A. First, a greater magnitude of inhibition was observed in the $250 \mathrm{SOA}$ condition in the pilot study, though the effect was not statistically reliable. Second, the robust inhibitory effect observed for both HCD and LCD targets in the 450 SOA condition in Experiment 1 was not observed in the pilot 
Table 19. Comparison of Experiment 1 and Pilot 2.A. word-target data. Facil (neutral minus related), Inhib (neutral minus unrelated) and Overall (unrelated minus related).

\begin{tabular}{|c|c|c|c|c|c|c|c|c|c|c|c|c|}
\hline \multirow{2}{*}{\multicolumn{2}{|c|}{ Priming Effects: }} & \multicolumn{3}{|c|}{$\begin{array}{l}\text { High and Low } \\
\text { Dominance }\end{array}$} & . & \multicolumn{3}{|c|}{$\begin{array}{c}\text { High } \\
\text { Dominance }\end{array}$} & . & \multicolumn{3}{|c|}{$\begin{array}{l}\text { Low } \\
\text { Dominance }\end{array}$} \\
\hline & & Facil & $\underline{\text { Inhib }}$ & Overall & . & $\underline{\text { Facil }}$ & $\underline{\text { Inhib }}$ & Qverall & . & Facil & Inhib & Overall \\
\hline & $\underline{\text { SOA }}$ & & & & . & & & & . & & & \\
\hline Exp 1: & 250 & $+57^{\mathrm{a}}$ & +01 & $+56^{\mathrm{a}}$ & . & $+44^{a}$ & -17 & $+62^{\mathrm{a}}$ & . & $+69^{\mathrm{a}}$ & +18 & $+51^{\mathrm{d}}$ \\
\hline Pilot 2. A.: & 250 & $+46^{8}$ & -30 & $+76^{d}$ & . & $+53^{\mathrm{h}}$ & -33 & $+86^{\mathrm{a}}$ & • & +38 & -27 & $+66^{\mathrm{h}}$ \\
\hline Exp 1: & 450 & +15 & $-67^{a}$ & $+83^{a}$ & . & +25 & $-58^{a}$ & $+83^{a}$ & & +06 & $-77^{\mathrm{a}}$ & $+83^{a}$ \\
\hline Pilot 2. A.: & 450 & +23 & -31 & $+54^{d}$ & . & $+42^{c}$ & -23 & $+65^{\mathrm{a}}$ & . & +02 & -40 & +42 \\
\hline
\end{tabular}

Note: Priming effects without superscript are not significant by either analysis. ${ }^{a}$ Significant at the .01 level by both the subject and item analysis. 'Significant at the .05 level by both the subject and item analysis. 'Significant at the .01 level by the subject analysis and at the .05 level by the item analysis. 'Significant at the .05 level by the subject analysis and at the .01 level by the item analysis. ${ }^{\mathrm{e}}$ Significant at the .05 level by the subject analysis but not significant by the item analysis. ${ }^{\mathrm{f}}$ Significant at the .01 level by the subject analysis but not significant by the item analysis. ${ }^{8}$ Significant at the .01 level by the item analysis but not significant by the subject analysis. 'Significant at the .05 level by the item analysis but not significant by the subject analysis. -Main effect for priming was significant, but individual differences between means did not reach significant level with Tukey post hoc analysis. 
data. Third, a significant facilitation effect was observed for HCD targets in the 450 SOA condition that was not observed in the Experiment 1 data.

\section{Pilot Study 2.B}

\section{Methods}

The purpose of this pilot study was to determine if the results obtained in the $250 \mathrm{~ms}$ and $450 \mathrm{~ms}$ SOA conditions in Experiment 1 could be replicated using a within subjects design. Although only six subjects were required for Pilot Study 2.B. all 12 subjects who participated in Pilot 2.A. were asked to return for a second session. Experimental sessions were spaced 1 week apart. Stimulus list versions and SOA conditions were counterbalanced across sessions such that each subject performed the task once with each SOA condition, but with different list versions. This counterbalancing is illustrated in Table 20. As noted in the table, subjects were assigned to one of two blocks. Although data from only six subjects were required, scheduling all 12 subjects provided a safeguard in case some subjects failed to return for the second session, The data from those subjects scheduled to Block 2 were arbitrarily selected for analysis. Otherwise, the stimulus materials and procedures were identical to those used in Experiment 1.

\section{$\underline{\text { Results }}$}

Reaction Time Analysis.

The mean reaction times (RTs) for correct responses to word targets in each of the three priming conditions (related, unrelated, and neutral), the two dominance conditions (HCD and LCD), and each SOA condition, are presented in the upper half of 
Table 20. Counterbalancing of SOA conditions and list versions for Pilot 2.B.

\begin{tabular}{lll}
\hline & Session 1 & Session II \\
\hline & & \\
& $250 \mathrm{~A}$ & $450 \mathrm{~F}$ \\
Block 1 & $250 \mathrm{C}$ & $450 \mathrm{~B}$ \\
& $250 \mathrm{E}$ & $450 \mathrm{D}$ \\
& $450 \mathrm{~B}$ & $250 \mathrm{E}$ \\
& $450 \mathrm{D}$ & $250 \mathrm{~A}$ \\
& $450 \mathrm{~F}$ & $250 \mathrm{C}$ \\
& & \\
& $250 \mathrm{~B}$ & $450 \mathrm{E}$ \\
& $250 \mathrm{D}$ & $450 \mathrm{~A}$ \\
Block 2 & $250 \mathrm{~F}$ & $450 \mathrm{C}$ \\
& $450 \mathrm{~A}$ & $250 \mathrm{~F}$ \\
& $450 \mathrm{C}$ & $250 \mathrm{~B}$ \\
& $450 \mathrm{E}$ & $250 \mathrm{D}$ \\
& \\
\hline
\end{tabular}


Tabie 21. Differences between these means are reported in the lower half of the table. No individual reaction times exceeded the $2000 \mathrm{~ms}$ cutoff used in the prior studies. To reduce variability in the data, each subject's data was cleaned individually. Missing values (errors) and extreme values (RTs $+/-2 \mathrm{SD}$ from the subject's mean per condition) were replaced with the subject's mean RT for that condition. Missing value replacements accounted for $2.8 \%$ of the data and extreme value replacements accounted for $5.8 \%$ of the data.

A separate $2(\mathrm{SOA}) \times 3$ (prime) $\times 2$ (dominance) ANOVA was performed with subject means (averaged across items) and item means (averaged across subjects). For the subject mean analysis, all three factors were treated as within subject factors. For the item mean analysis, dominance served as a between items factor and SOA and prime served as within items factors.

The main effect for prime was reliable on both analyses $\left[F_{1}(2,10)=4.01, p<\right.$ $\left..05 ; \mathrm{F}_{2}(2,116)=5.97, \mathrm{p}<.004\right]$. As noted in the lower half of Table 21 , the +34 overall priming effect was significant with the item mean analysis [Tukey HSD $\mathrm{cv}=$ $32.93, \mathrm{p}<.05$ ], but not the subject mean [Tukey HSD cv $=46.37, \mathrm{p}<.05$ ]. The $+18 \mathrm{~ms}$ facilitation effect, and the $-16 \mathrm{~ms}$ inhibition effect were not significant. The main effect for dominance was also reliable on both analyses $\left[F_{1}(1,5)=15.91, p<.02 ; F_{2}(1,58)=\right.$ $60.45, \mathrm{p}<.001]$. This analysis indicates that, collapsed across SOA and prime conditions, the $71 \mathrm{~ms}$ difference in mean RT to HCD targets (524 ms) and the mean RT to LCD targets (595 ms) was statistically reliable. The main effect for SOA was not significant by either analysis $\left[F_{1}(1,5)=.026, p<.88 ; F_{2}(1,58)=1.11, p<.30\right]$. 
Table 21. Pilot 2.B. reaction time data summary. Mean reaction times in milliseconds to word-target pairs in all dominance, priming ( $\mathrm{R}=$-related, $\mathrm{U}=$ =unrelated, $\mathrm{N}=$ neutral, and SOA conditions; and priming effects for each SOA and dominance condition representing the differences between mean reaction times for each of the three priming conditions. Facil (neutral minus related), Inhib (neutral minus unrelated) and Overall (unrelated minus related).

\begin{tabular}{|c|c|c|c|c|c|c|c|c|c|c|c|c|c|c|}
\hline \multirow[b]{2}{*}{ Priming Condition: } & \multicolumn{2}{|c|}{ High and Low } & \multicolumn{2}{|c|}{ Dominance } & • & \multirow{2}{*}{$\begin{array}{l}\text { High } \\
\underline{\mathrm{R}}\end{array}$} & \multicolumn{2}{|c|}{ Dominance } & \multirow[b]{2}{*}{$\underline{\text { Mean }}$} & \multirow[b]{2}{*}{. } & \multirow{2}{*}{$\begin{array}{l}\text { Low } \\
\underline{\mathrm{R}}\end{array}$} & \multicolumn{2}{|c|}{ Dominance } & \multirow[b]{2}{*}{$\underline{\text { Mean }}$} \\
\hline & $\underline{\mathrm{R}}$ & $\underline{\mathrm{U}}$ & $\underline{\mathrm{N}}$ & $\underline{\text { Mean }}$ & . & & $\underline{\mathrm{U}}$ & $\underline{N}$ & & & & $\underline{U}$ & $\underline{N}$ & \\
\hline SOA & & & & & & & & & & & & & & \\
\hline 250 & 543 & 562 & 559 & 555 & & 494 & 546 & 529 & 523 & . & 593 & 578 & 590 & 587 \\
\hline 450 & 541 & 589 & 561 & 563 & . & 486 & 561 & 525 & 524 & . & 595 & 617 & 596 & 603 \\
\hline Mean: & 542 & 576 & 560 & $\underline{560}$ & & 490 & 553 & 527 & $\underline{524}$ & . & 594 & 598 & 593 & $\underline{595}$ \\
\hline Priming Effects: & $\underline{\text { Facil }}$ & Inhib & \multicolumn{2}{|c|}{ Overall } & & $\underline{\text { Facil }}$ & Inhib & \multicolumn{2}{|c|}{ Qverall } & & $\underline{\text { Facil }}$ & $\underline{\text { Inhib }}$ & \multicolumn{2}{|c|}{ Overall } \\
\hline \multicolumn{15}{|l|}{ SOA } \\
\hline 250 & +16 & -03 & \multicolumn{2}{|l|}{+19} & & +35 & -17 & \multicolumn{2}{|l|}{+52} & . & -02 & -11 & \multicolumn{2}{|l|}{-13} \\
\hline 450 & +20 & -28 & \multicolumn{2}{|l|}{$+49^{\mathrm{a}}$} & & $+39^{\mathrm{a}}$ & -36 & \multicolumn{2}{|l|}{$+75^{\mathrm{a}}$} & . & +01 & -22 & \multicolumn{2}{|l|}{+22} \\
\hline Mean: & +18 & -16 & \multicolumn{2}{|l|}{$+34^{\mathrm{h}}$} & & $+37^{\mathrm{a}}$ & $-26^{c}$ & \multicolumn{2}{|l|}{$+63^{\mathrm{a}}$} & . & -01 & -05 & \multicolumn{2}{|l|}{+05} \\
\hline
\end{tabular}

Note: Priming effects without superscript are not significant by either analysis. ${ }^{2}$ Significant at the .01 level by both the subject and item analysis. 'Significant at the .05 level by both the subject and item analysis. 'Significant at the .01 level by the subject analysis and at the .05 level by the item analysis. 'Significant at the .05 level by the subject analysis and at the .01 level by the item analysis. ' Significant at the .05 level by the subject analysis but not significant by the item analysis. 'Significant at the .01 level by the subject analysis but not significant by the item analysis. ${ }^{8}$ Significant at the .01 level by the item analysis but not significant by the subject analysis. "Significant at the .05 level by the item analysis but not significant by the subject analysis. - Main effect for priming was significant, but individual differences between means did not reach significant level with Tukey post hoc analysis. 
The prime by dominance interaction was significant by both the subject and the item analysis $\left[F_{1}(1,10)=4.52, p<.04 ; F_{2}(2,116)=4.69, p<.01\right]$. To explore this interaction, separate one-way ANOVAs for the mean RTs (averaged across SOAs) obtained in the three priming conditions were performed for each of the two dominance conditions. These means are reported on the last line of the last two panels of Table 21 . The priming effects are reported in the lower half of the table. A main effect for priming was observed for the HCD condition $\left[F_{1}(2,10)=25.08, p<.001 ; F_{2}(2,58)=\right.$ $11.50, p<.001]$, but not the LCD condition $\left[F_{1}(2,10)=25.08, p<.001 ; F_{2}(2,58)=\right.$ $11.50, \mathrm{p}<.001]$, with both the subject and item mean analysis. The results of Tukey post hoc analyses of the HCD priming effects are indicated in Table 21 . The $+63 \mathrm{~ms}$ overall priming effect, the $+37 \mathrm{~ms}$ facilitation effect, and the $-26 \mathrm{~ms}$ inhibition effect were statistically significant by both the subject [Tukey HSD cv $=17.46, \mathrm{p}<.05 ; \mathrm{cv}=$ 23.72, $\mathrm{p}<.01$ ] and item mean analysis [Tukey HSD $\mathrm{cv}=22.61, \mathrm{p}<.05 ; \mathrm{cv}=28.46, \mathrm{p}<$ $.01]$.

In order to determine whether the facilitation, inhibition, and overall priming effects were significant in each of the individual SOA/dominance conditions, a separate one-way ANOVA for subject and item mean RTs obtained in the three priming conditions was performed separately for each of the individual SOA/dominance conditions. These means are reported in the upper half of the last two panels of Table 21 , and the differences between the means are reported in the lower half of the table. For those conditions in which a main effect of priming was observed, post hoc analyses were performed to determine the significance levels of the differences between the 
means. Relevant statistics from these analyses are reported in Table 22. As expected based on the prime by dominance interaction observed with the 3-way ANOVA, priming was not significant for LCD targets in either the 250 or the 450 SOA conditions. As shown in Table 22, priming was significant for HCD targets in the 450 SOA condition, by both the subject and item mean analysis. In the 250 SOA condition, priming for HCD targets approached significance with the item analysis, but not the subject analysis. Significance levels for the individual priming effects are indicated by superscript in Table 21 . The only individual priming effects that were statistically reliable were the $+39 \mathrm{~ms}$ facilitation, and the $+75 \mathrm{~ms}$ overall priming effect for HCD targets in the 450 SOA condition.

\section{Error Analysis}

More errors occurred in response to LCD word targets (13) than in response to HCD word targets (2). Also, the greatest number of errors occurred in the unrelated condition (10), and more errors were made in the neutral condition (3) than in the related condition (2). Since errors only account for $2.8 \%$ of the word target data, and, in general, support the reaction time data, they were not analyzed further.

\section{Discussion}

As in Experiment 1 and the previous pilot studies, responses to HCD targets were faster and more accurate than response to LCD target. Thus this dominance/frequency effect appears to be quite robust, and may be replicated with either between subjects or within subjects designs. With few exceptions however, the 
Table 22. Results of one-way ANOVAs for mean RTs obtained in the the three priming conditions for each SOA/dominance condition in Pilot 2.B.

\begin{tabular}{|c|c|c|c|c|c|c|c|c|}
\hline \multicolumn{9}{|c|}{$\underline{\text { HCD Targets }}$} \\
\hline \multicolumn{7}{|c|}{$\begin{array}{c}\text { Tukey CV } \\
q(3,10)\end{array}$} & \multicolumn{2}{|c|}{$\begin{array}{c}\text { Tukey CV } \\
q(3,58)\end{array}$} \\
\hline$\underline{\text { SOA }}$ & \multicolumn{2}{|c|}{$E_{1}(2.10 p$ value } & .05 & .01 & \multicolumn{2}{|c|}{$\mathrm{E}_{2}(2,58)$, p value } & .05 & .01 \\
\hline 250 & 2.90 & <. 110 & $\mathrm{n} / \mathrm{a}$ & $\mathrm{n} / \mathrm{a}$ & 3.00 & $<.06$ & $\mathrm{n} / \mathrm{a}$ & $\mathrm{n} / \mathrm{a}$ \\
\hline 450 & 11.19 & $<.004$ & 43.46 & 47.04 & 11.34 & $<.001$ & 37.84 & 47.64 \\
\hline \multicolumn{9}{|c|}{$\underline{\text { LCD Targets }}$} \\
\hline \multicolumn{7}{|c|}{$q(3,10)$} & \multicolumn{2}{|c|}{$\mathrm{q}(3,58)$} \\
\hline 250 & .09 & $<.91$ & $\mathrm{n} / \mathrm{a}$ & $\mathrm{n} / \mathrm{a}$ & .24 & $<.79$ & $\mathrm{n} / \mathrm{a}$ & $\mathrm{n} / \mathrm{a}$ \\
\hline 450 & 1.15 & $<.36$ & $\mathrm{n} / \mathrm{a}$ & $\mathrm{n} / \mathrm{a}$ & .45 & $<.64$ & $\mathrm{n} / \mathrm{a}$ & $\mathbf{n} / \mathbf{a}$ \\
\hline
\end{tabular}


results of Pilot 2.B fail to replicate the semantic priming effects observed in Experiment 1. These effects are presented together in Table 23 for comparison purposes. Unlike in Experiment 1, none of the individual priming effects observed in the $250 \mathrm{SOA}$ condition in the pilot data were statistically reliable. The $+35 \mathrm{~ms}$ facilitation effect and the +52 ms overall priming effect observed for HCD targets approximated the results obtained in Experiment 1. However, for LCD targets, there was absolutely no hint that responses were faster in the related condition in the 250 SOA condition, since the -02 $\mathrm{ms}$ facilitation effect and the $-13 \mathrm{~ms}$ overall priming effect indicate that responses were slower in the related priming condition than in the neutral and unrelated priming conditions. These results contrast with the significant facilitation and overall priming effects observed in the 250 SOA condition in Experiment 1 for both HCD and LCD targets.

In the 450 SOA condition, a significant overall priming effect was observed for HCD targets in both Experiment 1 and Pilot 2.B. However, unlike in Experiment 1, the pilot data yielded a significant facilitation effect for HCD targets, and no significant effects for LCD targets. Once again, the robust inhibitory effect observed in Experiment 1 was not replicated.

In summary, the manipulation of SOA within subjects, even across only two sessions, failed to yield a clear replication of the results obtained in Experiment 1. The most damaging aspect of the results obtained in Pilot 2.B is that few of the observed priming effects were statistically reliable. Furthermore, results obtained for LCD targets in the 250 SOA condition actually fell in the wrong direction. 
Table 23. Comparison of Experiment 1 and Pilot 2.B reaction time priming effects for word-target data. Facil (neutral minus related), Inhib (neutral minus unrelated) and Overall (unrelated minus related).

\begin{tabular}{|c|c|c|c|c|c|c|c|c|c|c|c|c|}
\hline \multirow{2}{*}{\multicolumn{2}{|c|}{ Priming Effects: }} & \multicolumn{3}{|c|}{$\begin{array}{l}\text { High and Low } \\
\text { Dominance }\end{array}$} & . & \multicolumn{3}{|c|}{$\begin{array}{c}\text { High } \\
\text { Dominance }\end{array}$} & • & \multicolumn{3}{|c|}{$\begin{array}{c}\text { Low } \\
\text { Dominance }\end{array}$} \\
\hline & & $\underline{\text { Facil }}$ & Inhib & Overall & . & $\underline{\text { Facil }}$ & $\underline{\text { Inhib }}$ & Overall & . & $\underline{\text { Facil }}$ & Inhib & Qverall \\
\hline & $\underline{\mathrm{SOA}}$ & & & & & & & & . & & & \\
\hline Exp 1: & 250 & $+57^{\mathrm{a}}$ & +01 & $+56^{\mathrm{a}}$ & . & $+44^{\mathrm{a}}$ & -17 & $+62^{\mathrm{a}}$ & . & $+69^{a}$ & +18 & $+51^{d}$ \\
\hline Pilot 2. B.: & 250 & +16 & -03 & +19 & . & +35 & -17 & +52 & . & -02 & -11 & -13 \\
\hline Exp 1: & 450 & +15 & $-67^{a}$ & $+83^{a}$ & . & +25 & $-58^{a}$ & $+83^{a}$ & . & +06 & $-77^{\mathrm{a}}$ & $+83^{a}$ \\
\hline Pilot 2. B.: & 450 & +20 & -28 & $+49 \mathrm{a}$ & . & $+39_{a}$ & -36 & $+75_{a}$ & . & +01 & -22 & +22 \\
\hline
\end{tabular}

Note: Priming effects without superscript are not significant by either analysis. ${ }^{\text {a }}$ Significant at the .01 level by both the subject and item analysis. 'Significant at the .05 level by both the subject and item analysis. 'Significant at the .01 level by the subject analysis and at the .05 level by the item analysis. 'Significant at the .05 level by the subject analysis and at the .01 level by the item analysis. 'Significant at the .05 level by the subject analysis but not significant by the item analysis. 'Significant at the .01 level by the subject analysis but not significant by the item analysis. ${ }^{\circ}$ Significant at the .01 level by the item analysis but not significant by the subject analysis. "Significant at the .05 level by the item analysis but not significant by the subject analysis. - Main effect for priming was significant, but individual differences between means did not reach significant level with Tukey post hoc analysis. 
In contrast, the results of Pilot 2.A. suggest that standard priming effects can be replicated using a small number of subjects and a between subjects design, although not all the results conformed to those of Experiment 1. In the 250 SOA condition, the same pattern of facilitation without significant inhibition for HCD and LCD targets that was replicated. Furthermore, an overall priming effect was observed in each SOA condition for both HCD and LCD targets, although the $+42 \mathrm{~ms}$ overall priming effect for LCD targets in the 450 SOA condition was not statistically reliable (see Table 19). However, in the 450 SOA condition a significant facilitation effect was observed for HCD targets that was not observed in the Experiment 1 data. Also, some inhibition was observed for both HCD and LCD targets in the 450 SOA condition, but in neither case was this effect statistically reliable. Thus, the results of Pilot 2.A. provided support for automatic processing in the 250 SOA condition, but less convincing evidence of strategic processing in the 450 SOA condition.

The limited availability of subjects with Broca's aphasia, and the questionable ability to replicate priming effects using a within-subjects design pose serious challenges to examining the impact of SOA on automatic versus strategic priming effects in this population. The question remains therefore, how to best address current hypotheses regarding automatic versus strategic processing in Broca's aphasia by examining priming in a single SOA condition. The 450 SOA condition seems a poor choice for two reasons. First, the inhibitory effects observed in the 450 SOA condition in Pilot 2.A. were not significant, and thus only weakly support the possiblity that subjects were using controlled processing. Second, and perhaps more important, 
convincing data has already been reported that supports the view that strategic processing in Broca's aphasia is abnormal (Bushell, 1996; Milberg et al., 1995). Therefore, an examination of priming in Broca's aphasics in the 450 SOA condition would not be particularly informative.

On the other hand, an examination of priming in the 250 SOA condition, using the materials described for Experiment 1, may address several of the hypotheses that have been made regarding automatic processing in Broca's aphasia. Recall that three claims have been made in this regard. First, several investigators have argued that automatic processing is intact in Broca's aphasia (Hagoort, 1997; Ostrin \& Tyler, 1993; Tyler et al., 1995). This claim is based on the observation that Broca's aphasics have consistently produced intact overall priming effects with the visual and auditory pairwise paradigms when short SOA/ISIs were used. Further support for this hypothesis would be garnered if in an experiment using a neutral control condition and a 250 SOA, Broca's aphasics demonstrate facilitation without inhibition.

A second opinion regarding the nature of automatic processing in Broca's aphasia is that automatic activation is intact, but operates "under a slower than normal rise time" (Prather, et al., 1997; Prather et al., 1992; Swinney et al., 1989). This view is based on data obtained with the cross-modal lexical decision paradigm demonstrating priming only for the most frequent interpretation of ambiguous words, and the listpriming paradigm demonstrating priming at longer ISIs than observed with normal subjects. Additional support for this hypothesis would be gained if Broca's aphasics were to demonstrate priming for $\mathrm{HCD}$ but not $\mathrm{LCD}$ targets in a 250 SOA condition. 
Such a finding would suggest that their automatic access to lexical items was intact, but that insufficient time was available to access the lower frequency/dominance targets.

Finally, Milberg et al. (1995) have argued that the level of automatic activation is reduced in Broca's aphasics, such that they are likely to display no facilitation in tasks not conducive to the use of strategies. Furthermore, they suggested that this impairment has previously been masked because under many conditions Broca's aphasics may overrely on the use of strategies. Recall that they observed facilitation without inhibition in a 150 ISI condition, but attributed the effect to controlled rather than automatic processing, claiming that their subjects were able to use strategies even in this short ISI condition. If indeed Broca's aphasics are overreliant on stategic processing, and are able to use strategies even at short SOAs, it is likely that given a stimulus set designed to induce the use of strategic processing both a facilitatory and an inhibitory effect would be observed. The materials used for the present series of experiments were designed specifically to encourage the use of strategic processing, by including both a high relatedness proportion and a high nonword ratio. Thus, the observation of significant facilitation and inhibition would be congruent with Milberg et al.'s hypothesis that priming by Broca's aphasics at short SOAs can be mediated by strategic rather than automatic processing. 


\section{Experiment 2: Broca's Aphasics and Age Matched Controls}

\section{$\underline{\text { Methods }}$}

\section{Procedure}

The purpose of this experiment was to determine whether individuals with Broca's aphasia would display the same pattern of facilitatory and inhibitory effects as normal subjects in a pairwise visual lexical decision task with a $250 \mathrm{~ms}$ prime-target SOA. Three subject groups were used, a young normal control group, an older agematched control group, and a group of individuals with Broca's aphasia. The stimulus materials and procedures were identical to those used in Experiment 1 with the exception that all three groups performed the task with the same $250 \mathrm{~ms}$ SOA. Once again, nonword data were not considered for analysis.

\section{Subjects}

A total of 6 subjects were included in each subject group. Data for the "young normal" control group were those obtained in the 250 SOA condition in Pilot 2.A. The "older normal" subjects were roughly matched by age $(+/-5$ years $)$ and education $(+/-2$ year) with the aphasic subjects. The mean age of the young normal subjects was 20 years, and the mean age of the old normal subjects was 57 years. All subjects were native, monolingual speakers of English, with at least a high school grade education. Subjects all demonstrated sufficient visual acuity (aided or unaided) to read stimulus words presented white on black, such as those used in the experiments. This was determined by having subjects match ten words printed on paper (black on white) to words presented on the computer screen (white on black). No subject had a history of 
psychiatric disorder, or drug or alcohol abuse. Persons with a history of neurological disorder other than that precipitating the diagnosis of aphasia in the aphasic subject group, were excluded.

All aphasic subjects had suffered a single stroke to the left hemisphere resulting in aphasia with markedly reduced verbal expression and right hemiparesis. They all demonstrated relatively spared reading ability for single words as determined by $80 \%$ or greater accuracy on the Word-Picture Matching Subtests of the Western Aphasia Battery. The diagnosis of aphasia subtype was determined by performance on the Western Aphasia Battery (WAB) (Kertesz, 1982). All aphasic subjects were a minimum of six months post onset at the time language testing was conducted. Table 24 shows a summary of the patients' aphasia diagnosis, WAB scores, age, gender, education, time postonset, and lesion localization. The critical features that distinguish Broca's aphasia from other subtypes of aphasia are 1) nonfluent, effortful and poorly articulated verbal expression consisting primarily of short phrases, 2) a poor ability to repeat, and 3) relatively well preserved auditory comprehension. Two subjects were included whose verbal expression skills had evolved such that they no longer met the clinical diagnosis of Broca's aphasia. However, medical records and history information obtained from the subjects clearly indicated that their behavioral profile was consistent with a diagnosis of Broca's aphasia earlier in their course of recovery. Nonetheless, any claims made regarding the performance of Broca's aphasics, based on the data obtained in this study, must be made with some degree of caution. With the exception of the aphasic subject, JB, all subjects were right-handed. The mean age of 
Table 24. Aphasia subject summary.

\begin{tabular}{|c|c|c|c|c|c|c|c|c|c|c|c|}
\hline \multirow{3}{*}{$\begin{array}{l}\text { Subj } \\
\mathrm{KN}\end{array}$} & \multirow{3}{*}{$\begin{array}{l}\text { Aphasia } \\
\text { Subtype }\end{array}$} & \multirow{2}{*}{\multicolumn{2}{|c|}{ Gender/Age }} & \multirow{3}{*}{$\begin{array}{l}\begin{array}{l}\text { Time } \\
\text { Postonset }\end{array} \\
12 \mathrm{mo}\end{array}$} & \multicolumn{6}{|c|}{ Western Aphasia Battey Scores } & \multirow{3}{*}{$\begin{array}{l}\text { Lesion Description } \\
\text { Ischemic infarction in left } \\
\text { hemisphere and basal ganglia } \\
\text { w/o hemorrhage }\end{array}$} \\
\hline & & & & & $\begin{array}{r}F L \\
(0-4)\end{array}$ & $\begin{array}{c}A C \\
(4-10)\end{array}$ & $\begin{array}{c}\text { Rep } \\
(0-7.9)\end{array}$ & $\begin{array}{l}\mathrm{Nmg} \\
(0-8)^{*}\end{array}$ & $\mathrm{AQ}$ & Education & \\
\hline & & $\mathrm{F}$ & 46 & & 1 & 7.95 & 2.6 & 3.0 & 39.10 & highschool & \\
\hline $\mathrm{PP}$ & Broca's & $\mathrm{F}$ & 52 & $11 \mathrm{mo}$ & 1 & 6.30 & .20 & .20 & 21.40 & highschool & $\begin{array}{l}\text { Left frontoparietal and basal } \\
\text { ganglia }\end{array}$ \\
\hline $\mathrm{KB}$ & Broca's & $\mathrm{F}$ & 48 & $6 \mathrm{yr}, 5 \mathrm{mo}$ & 2 & 7.85 & 1.6 & 3.50 & 35.90 & highschool & $\begin{array}{l}\text { Left infarction involving the } \\
\text { frontal and parietal lobes and } \\
\text { possibly segments of the } \\
\text { temporal lobe. }\end{array}$ \\
\hline JB & Broca's & $\mathbf{M}$ & 59 & $13 \mathrm{yr}, 2 \mathrm{mo}$ & 2 & 8.05 & 1.20 & 6.40 & 45.30 & 4 yr college & $\begin{array}{l}\text { L frontal hemorrhage } \\
\text { requiring surgical evacuation }\end{array}$ \\
\hline FT & Anomic & $\mathrm{M}$ & 74 & $3 \mathrm{yr}, 8 \mathrm{mo}$ & 6 & 8.85 & 9.60 & 8.30 & 81.50 & highschool & $\begin{array}{l}\text { Infarct involving the } \\
\text { subcortical white matter of } \\
\text { the left frontoparietal region }\end{array}$ \\
\hline DR & Conduction & M & 61 & $5 \mathrm{yr}, 1 \mathrm{mo}$ & 5 & 8.05 & 5.60 & 6.30 & 59.90 & 2 yr college & $\begin{array}{l}\text { Left internal carotid artery } \\
\text { infarct }\end{array}$ \\
\hline
\end{tabular}


the aphasic subjects was 56 years. Aphasic subjects and age-matched controls were paid $\$ 25$ for their participation in the experiment. Aphasic subjects were also paid $\$ 25$ for their participation in the language evaluation. ${ }^{3}$

\section{Results}

\section{Reaction Time Analysis}

The mean reaction times (RTs) for correct responses to word targets in each of the three priming conditions (related, unrelated, and neutral) and each of the two dominance conditions (HCD and LCD) are presented for each subject group in the upper half of Table 25. Differences between these means are reported as priming effects in the upper half of Table 26. Before these mean values were calculated, all RTs $\geq 2000 \mathrm{~ms}$ were converted to errors. For the aphasic subjects, these long latencies occurred on $3.6 \%$ of the correct word target responses. For the young and older control groups, no correct RTs exceeded this cutoff. To further reduce variability in the data, each subject's data was cleaned individually. Missing values (errors) and extreme values (RTs +/- 2 SD from the subject's mean per condition) were replaced with the subject's mean RT for that condition. For the aphasic subjects, missing value replacements accounted for $19.72 \%$ of the data and extreme value replacements accounted for $1.67 \%$ of the data. For the older controls, missing value replacements accounted for $1.94 \%$ of the data and extreme value replacements accounted for $4.72 \%$ of the data. For the younger controls, missing value replacements accounted for $3.06 \%$ of the data and extreme value replacements accounted for $3.61 \%$ of the data. 


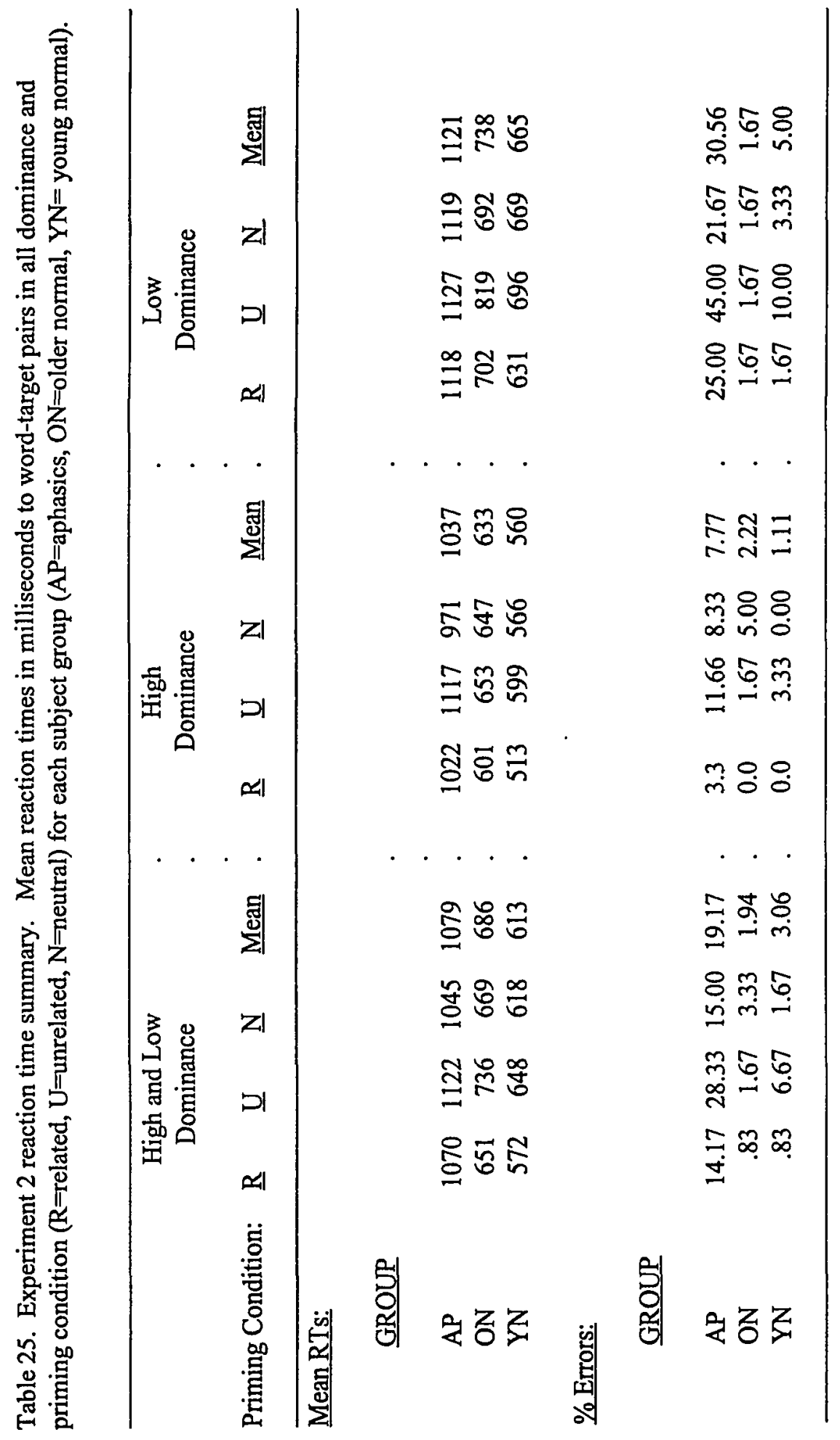

123

Reproduced with permission of the copyright owner. Further reproduction prohibited without permission. 
Table 26. Experiment 2 priming effects summary. Reaction time priming effeccts are presented in the top half of the table. Error rate priming effects are presented in the bottom half of the table. $(\mathrm{AP}=$ aphasics, $\mathrm{ON}=$ older normal, $\mathrm{YN}=$ young normal, Facil $=$ neutral minus related, Inhib $=$ neutral minus unrelated, and Overall $=$ unrelated minus related)

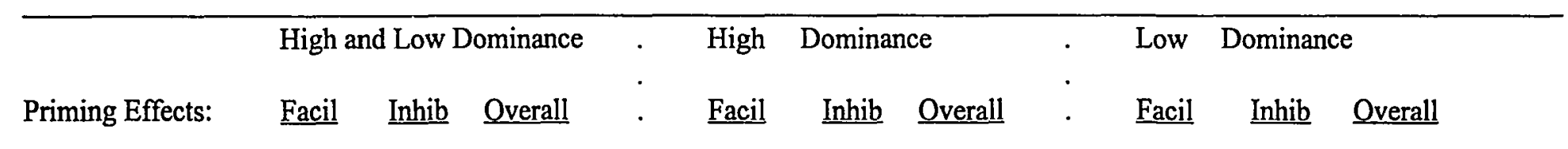

RTs:

GROUP

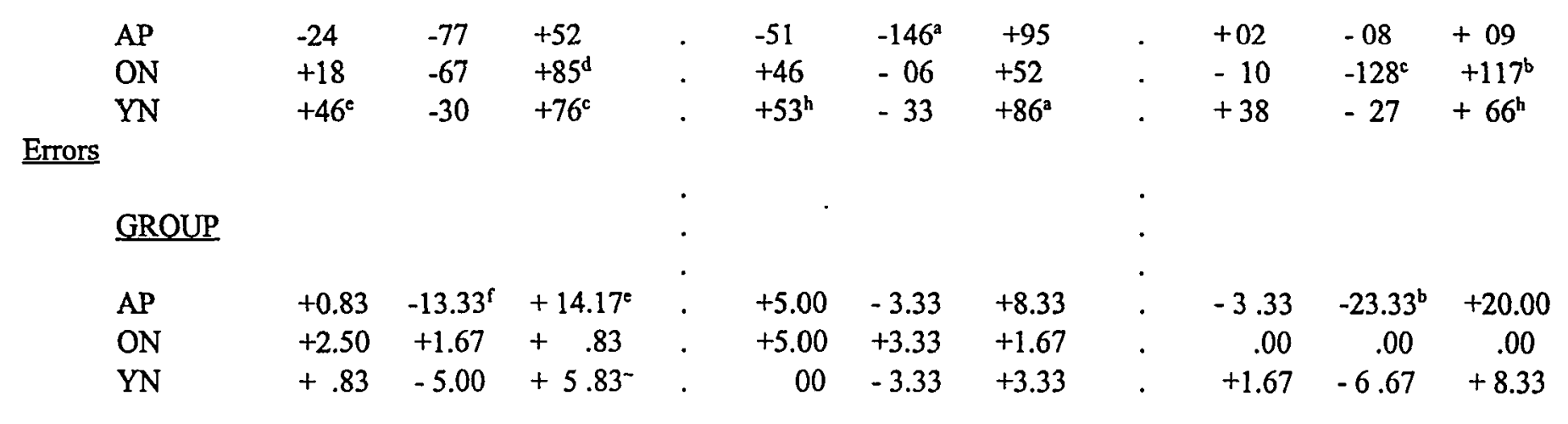

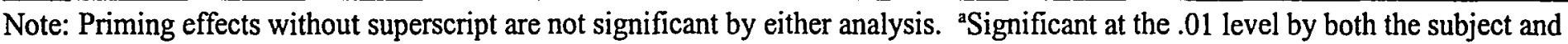
item analysis. 'Significant at the .05 level by both the subject and item analysis. ' ${ }^{c}$ Significant at the .05 level by the subject analysis and at the .01 level by the item analysis. ${ }^{d}$ Significant at the .05 level by the subject analysis but not significant by the item analysis. 'Significant at the .01 level by the item analysis but not significant by the subject analysis. 'Significant at the .05 level by the item analysis but not significant by the subject analysis. -Main effect for priming was significant, but individual differences between means did not reach significant level with Tukey post hoc analysis. 
A separate 3(group) x 3(prime) x 2(dominance) ANOVA was performed with subject means (averaged across items) and item means (averaged across subjects). For the subject mean analysis, group was treated as a between subjects factor, and prime and dominance served as within subjects factors. For the item mean analysis dominance served as a between items factor and group and prime served as within items factors.

The main effect for group was reliable on both analyses $\left[F_{1}(2,15)=23.41, p<\right.$ $.001 ; \mathrm{F}_{2}(2,116)=10.50, \mathrm{p}<.001 \mathrm{]}$. The aphasic subjects' mean RT $(1079 \mathrm{~ms})$ was 466 $\mathrm{ms}$ slower than that of the young control subjects $(613 \mathrm{~ms})$ and $393 \mathrm{~ms}$ slower than that of the older control subjects $(686 \mathrm{~ms})$. Tukey post hoc analyses indicated that these differences were statistically reliable [Tukey $\mathrm{HSD}_{1} \mathrm{cv}=355, \mathrm{p}<.01$; Tukey $\mathrm{HSD}_{2} \mathrm{cv}=$ $85, \mathrm{p}<.01]$. The $686 \mathrm{~ms}$ mean RT for old normal controls was $73 \mathrm{~ms}$ slower than the $613 \mathrm{~ms}$ mean RT for young normal controls. This difference was significant by the item analysis only [Tukey $\mathrm{HSD}_{1} \mathrm{cv}=269, \mathrm{p}<.05$; Tukey $\mathrm{HSD}_{2} \mathrm{cv}=68, \mathrm{p}<.05$ ]

The main effect for prime was reliable on both analyses $\left[F_{1}(2,30)=8.46, p<\right.$ $\left..002 ; F_{2}(2,116)=485.722, p<.001\right]$, as was the main effect for dominance $\left[F_{1}(1,15)=\right.$ $\left.48.81, p<.001 ; F_{2}(1,58)=41.56, p<.001\right]$. Across the three groups, responses to HCD targets were $98 \mathrm{~ms}$ faster than responses to LCD targets. A Tukey post hoc analysis of the main effect for prime was not conducted since priming effects for the three groups combined was of little interest.

A three way interaction between group, prime, and dominance approached statistical significance with the subject analysis $\left[F_{1}(4,30=2.23, p<.09]\right.$ and was significant by the item analysis $\left[\mathrm{F}_{2}(4,232)=3.15, \mathrm{p}<.02\right]$. To investigate the nature of 
this interaction, a 3 (prime) $\times 2$ (dominance) ANOVA was performed for each subject group.

Aphasic Subjects For the aphasic subjects, the only significant main effect observed was for the dominance factor, which approached statistical significance with the subject analysis $\left[F_{1}(1,5)=5.19, p<.07\right]$ and was statistically reliable with the item analysis $\left.F_{2}(1,58)=5.95, p<.02\right]$. This result indicates that the $84 \mathrm{~ms}$ difference in aphasics subjects' mean RT to HCD targets (1037 ms) was reliably faster than their mean RT to LCD targets $(1121 \mathrm{~ms})$. The main effect for prime $\left[F_{1}(2,10)=1.53, p<\right.$ $\left..26 ; F_{2}(2,116)=2.21, p<.12\right]$ and the prime by dominance interaction $\left[F_{1}(2,10)=\right.$ $\left.1.54, \mathrm{p}<.26 ; \mathrm{F}_{2}(2,116)=1.76, \mathrm{p}<.18\right]$ were not significant by either the subject or item mean analysis. Therefore, the $-24 \mathrm{~ms}$ facilitation effect, the $-77 \mathrm{~ms}$ inhibition effect, and the $+52 \mathrm{~ms}$ overall priming effects reported in the upper half of Table 26 were not statistically reliable. The fact that none of these effects reached statistical significance is likely due to the high variability observed with this group (subject mean $\mathrm{SD}=184$, item mean $\mathrm{SD}=139$ ).

A one-way ANOVA of the mean RTs obtained in each priming condition was performed separately for HCD and LCD targets to determine if any of the individual priming effects in either of these dominance conditions were significant. This analysis yielded a main effect for priming for $\mathrm{HCD}$ targets $\left[\mathrm{F}_{1}(2,10)=6.61, \mathrm{p}<.02 ; \mathrm{F}_{2}(2,58)=\right.$ $3.94, \mathrm{p}<.03]$, but not $L C D$ targets $\left[F_{1}(2,10)=.01, p<.99 ; F_{2}(2,58)=.02, p<.98\right]$, by both the subject and item mean analysis. Tukey post hoc analysis of the differences between the means (for HCD targets only) indicated that the $-146 \mathrm{~ms}$ inhibition effect 
was significant by both the subject and item mean analysis [Tukey $\mathrm{HSD}_{1} \mathrm{cv}=112, \mathrm{p}<$ $\left..05 ; \mathrm{HSD}_{2} \mathrm{cv}=127, \mathrm{p}<.05\right]$. This effect means that responses to targets in the unrelated priming condition were reliably slower than in the neutral priming condition. The negative value of the -51 facilitation effect indicates that responses to targets in the related priming condition were also slower than responses to targets in the neutral priming condition. However, this effect and the $+95 \mathrm{~ms}$ overall priming effect were not statistically reliable.

Old Normal Controls The 3 (prime) $\times 2$ (dominance) ANOVA for this group yielded a main effect for priming by both the subject and item analysis $\left[F_{1}(2,10)=\right.$ $\left.11.67, \mathrm{p}<.003 ; \mathrm{F}_{2}(2,58)=7.02, \mathrm{p}<.002\right]$. As noted in Table 26 , the $+85 \mathrm{~ms}$ overall priming effect was significant by the subject analysis but not the item analysis [Tukey $\left.\mathrm{HSD}_{\mathrm{I}} \mathrm{cv}=71.78, \mathrm{p}<.05 ; \mathrm{HSD}_{2} \mathrm{cv}=150.23, \mathrm{p}<.05\right]$. Neither the +18 ms facilitation effect, nor the -67 inhibition effect were significant. Variability for this group was considerably smaller than for the aphasic groups (subject mean $\mathrm{SD}=69$, item mean $\mathrm{SD}$ $=82$ ).

A main effect for dominance was also observed for the older controls $\left[F_{1}(1,5)=\right.$ $\left.146.68, \mathrm{p}<.001 ; \mathrm{F}_{2}(1,58)=40.36, \mathrm{p}<.001\right]$. This result indicates that the $105 \mathrm{~ms}$ difference in the older control's mean RT to HCD targets ( $633 \mathrm{~ms})$ was reliably faster than their mean RT to LCD targets (665 ms).

The two-way ANOVA also yielded a significant prime $\mathrm{x}$ dominance interaction that was significant by the item analysis, and approached significance with the subject analysis $\left[F_{1}(2,10)=3.05, p<.09 ; F_{2}(2,58)=3.24, p<.05\right]$. To investigate this 
interaction further, and to determine if any of the individual priming effects in either of the two dominance conditions were significant, a one-way ANOVA of the mean RTs obtained in each priming condition was performed separately for HCD and LCD targets. Priming for HCD targets was not quite signficant by either the subject or item mean analysis $\left[F_{1}(2,10)=3.04, p<.09 ; F_{2}(2,58)=2.49, p<.09\right]$. Thus the $+46 \mathrm{~ms}$ facilitation effect, the -06 inhibition effect, and the +52 overall priming effects reported in Table 26 for old normal controls in the HCD condition were not statistically reliable. Priming for LCD targets was significant by both the subject and item mean analysis $\left[\mathrm{F}_{1}\right.$ $\left.(2,10)=7.40, p<.01 ; F_{2}(2,58)=6.19, p<.005\right]$. A Tukey post hoc analysis of the differences between the means obtained for LCD targets indicated that the $-128 \mathrm{~ms}$ inhibition effect, and the -117 overall priming effect were significant by both the subject analysis and the item analysis [Tukey $\mathrm{HSD}_{1} \mathrm{cv}=101.19, \mathrm{p}<.05 ; \mathrm{HSD}_{2} \mathrm{cv}=96.93, \mathrm{p}<$ $.05, \mathrm{cv}=122.02, \mathrm{p}<.01]$. The $-10 \mathrm{~ms}$ facilitation effect was not significant. Notice again that the negative value of this facilitation effect indicates that responses to targets in the related condition were slower than responses to targets in the neutral condition.

Young Normal Subjects The reaction time data for the young normal controls was analyzed and reported in the results section for Pilot 2.A. To review the results of that analysis, averaged across the two dominance conditions, the +76 ms overall priming effect and the $+46 \mathrm{~ms}$ facilitation effect were significant, but the $-30 \mathrm{~ms}$ inhibition effect was not statistically reliable. For HCD targets, the +86 ms overall priming effect, and the $+53 \mathrm{~ms}$ facilitation effect were significant, but the $-33 \mathrm{~ms}$ inhibition effect was not reliable. For LCD targets, the +66 ms overall priming effect 
was significant, but neither the $+38 \mathrm{~ms}$ facilitation effect, nor the $-27 \mathrm{~ms}$ inhibition effect, were reliable. Significance levels for these effects are indicated by superscript in Table 26. For the young normals, variability was slightly higher than than of the older controls (subject mean $\mathrm{SD}=99$, item mean $\mathrm{SD}=84$ ).

Summary of the RT Results Each group of subjects was reliably faster in their response to HCD targets compared to $\mathrm{LCD}$ targets. This again represents the dominance/frequency effect that has been reported throughout the series of studies reported here. For the HCD targets, all three groups were faster in their responses to targets following a related prime than to those following an unrelated prime, although this overall priming effect was reliable only for the young control group. For both the control groups, responses were faster to targets following a related prime than to those following a neutral prime, although this facilitation effect was not reliable for the old normal controls. In contrast, the aphasic subjects' mean RT to HCD targets following related primes was $51 \mathrm{~ms}$ slower than their mean RT to targets following a neutral prime. Also, whereas the control groups each displayed an insignificant amount of inhibition, the aphasic subjects displayed a robust and statistically reliable inhibition effect $(-146 \mathrm{~ms})$

For LCD targets both the old normal controls and the young normal controls displayed a significant overall priming effect. However, the contribution of facilitation and inhibition to this effect was quite different for the two groups. Unlike the young normal controls, the old normal controls displayed a large and significant inhibitory effect. Also, unlike the young normal controls, the old normal controls displayed no 
hint of a facilitation effect, since their mean RT to targets following a related prime was $10 \mathrm{~ms}$ slower than their mean RT to targets following a neutral prime. For the young normal controls, the facilitation effect was larger than the inhibitory effect, reflecting a pattern of facilitation dominance, although neither of these individual effects were statistically reliable. For the aphasics subjects all of the observed reaction time priming effects for LCD targets were negligible. Since this likely reflects the large proportion of errors they made in response to LCD targets (31\%), a full analysis of the error data will be reported below.

Error Analysis

The mean percentage of errors for incorrect responses to word targets in each of the three priming conditions (related, unrelated, and neutral) and each of the two dominance conditions (HCD and LCD) are presented for each subject group in the lower half of Table 25. Differences between these mean percentages are reported as priming effects in the lower half of Table 26. The mean percentages of errors per condition were submitted to the same statistical analyses as were the reaction time data. The 3(group) x 3(prime) $\times 2$ (dominance) ANOVA yielded a main effect for group that was reliable on both analyses $\left[F_{1}(2,15)=10.63, p<.002 ; F_{2}(2,116)=7.92, p<.002\right]$. The aphasic subjects' made $17.23 \%$ more errors than the old control subjects and 16.11 $\%$ more errors than the young control subjects. Tukey post hoc analyses indicated that these differences were statistically reliable [Tukey $\mathrm{HSD}_{1} \mathrm{cv}=20.23, \mathrm{p}<.01$; Tukey $\left.\mathrm{HSD}_{2} \mathrm{cv}=8.27, \mathrm{p}<.01\right]$. The difference in mean percentage of errors produced by the two control groups was not statistically significant. 
The main effect for prime was reliable on both analyses $\left[F_{1}(2,30)=6.51, p<\right.$ $\left..005 ; F_{2}(2,116)=45.14, p<.001\right]$, as was the main effect for dominance $\left[F_{1}(1,15)=\right.$ $\left.17.62, \mathrm{p}<.002 ; \mathrm{F}_{2}(1,58)=32.34, \mathrm{p}<.001\right]$. A significantly greater number of errors were made in response to LCD targets $(12.41 \%)$ than in response to HCD targets $(3.7 \%)$

Both the subject analysis and the item analysis yielded a prime by dominance interaction $\left[F_{1}(2,30)=3.32, p<.05 ; F_{2}(2,116)=23.93, p<.001\right]$, and a group by dominance interaction $\left[F_{1}(2,15)=11.90, p<.002 ; F_{2}(2,116)=5.50, p<.006\right]$. In addition, the subject analysis yielded a prime $\mathrm{x}$ group analysis that approached statistical significance with the item analysis $\left[F_{1}(4,30)=2.75, p<.05 ; F_{2}(4,232)=\right.$ $2.11, \mathrm{p}<.08$ ]. To investigate the nature of these interactions, a 3 (prime) $\times 2$ (dominance) ANOVA was performed for each subject group.

Aphasic Subjects Both the subject mean and the item mean analysis yielded a main effect for prime for the aphasic subjects $\left[F_{1}(2,10)=4.42, p<.04 ; F_{2}(2,116)=\right.$ $3.84, \mathrm{p}<.02]$. A Tukey post hoc analysis of the differences between the mean error rates (averaged across dominance conditions) indicated that the -13.33 inhibition effect and the +14.17 overall priming effect reported in Table 26 were significant by the item analysis but not the subject analysis [Tukey $\mathrm{HSD}_{1} \mathrm{cv}=20.76, \mathrm{p}<.05$; Tukey $\mathrm{HSD}_{2} \mathrm{cv}=$ 11.16, $\mathrm{p}<.05, \mathrm{cv}=13.94, \mathrm{p}<.01]$. The +.83 facilitation effect was not significant. These results indicate that the aphasic subjects made significantly more errors in response to targets preceded by unrelated primes in comparison to those targets 
preceded by either a neutral prime (inhibition effect) or a related prime (overall priming effect).

The main effect for dominance was also significant by both analyses $\left[F_{1}(1,5)=\right.$ 18.23, $\left.\mathrm{p}<.009 ; \mathrm{F}_{2}(1,58)=33.94, \mathrm{p}<.001\right]$. Aphasic subjects made $30.56 \%$ errors in response to LCD targets, which was significantly greater than the $7.77 \%$ errors they made in response to $\mathrm{HCD}$ targets.

Although the prime by dominance interaction was not significant, a one-way ANOVA of the mean error rates obtained in each priming condition was performed separately for $\mathrm{HCD}$ and LCD targets to determine if the significance levels of the individual priming effects in each of the two dominance conditions. This analysis yielded a main effect for priming for $L C D$ targets $\left[F_{1}(2,10)=5.66, p<.02 ; F_{2}(2,58)=\right.$ $4.46, \mathrm{p}<.02]$, but not $\mathrm{HCD}$ targets $\left[\mathrm{F}_{1}(2,10)=.94, \mathrm{p}<.42 ; \mathrm{F}_{2}(2,58)=.77, \mathrm{p}<.47\right]$, by both the subject and item mean analysis. Tukey post hoc analysis of the differences between the mean error rates for LCD targets indicated that the $+20.00 \%$ overall priming effect approached significance, and the $-23.33 \%$ inhibition effect was significant, by both the subject and item mean analysis [Tukey $\mathrm{HSD}_{1} \mathrm{cv}=20.56, \mathrm{p}<$ $\left..05 ; \mathrm{HSD}_{2} \mathrm{cv}=20.30, \mathrm{p}<.05\right]$. This effect means that the aphasic subjects made significantly more errors in response to targets in the unrelated priming condition than in the unrelated or the neutral priming condition. Notice that the negative value of the $-3.33 \%$ facilitation effect indicates that more errors were made in response to targets in the related priming condition than in the neutral priming condition. However, this effect was not statistically reliable. 
Old Normal Controls The 3 (prime) $\times 2$ (dominance) ANOVA of mean error rates for this group yielded no significant main effects or interactions. As noted previously, the old normal controls made fewer than $2 \%$ errors across the two dominance conditions combined. Given so few errors, separate oneway ANOVAS to determine significance levels for the HCD and LCD targets were not performed.

Young Normal Controls The 3 (prime) $\times 2$ (dominance) ANOVA of mean error rates for the young normal control group yielded a main effect for prime by the subject analysis but not the item analysis $\left[F_{1}(2,10)=4.06, p<.05 ; F_{2}(2,116)=1.00, p<.37\right]$. The main effect for dominance, and the prime by dominance interaction were not significant by either analysis. A Tukey post hoc analysis of the differences between (subject) mean error rates in the three priming conditions (averaged across dominance) indicated that none of the individual priming effects were significant. These results are likely due to the relatively few errors ( $3.06 \%)$ produced across the two dominance conditions. Given so few errors, separate oneway ANOVAS to determine significance levels for the HCD and LCD targets were not performed.

Summary of the Error Results The aphasic subjects, but neither of the control group subjects, made significantly greater errors in their response to LCD targets compared to HCD targets. None of the individual priming effects were significant in the HCD condition, for any of the subject groups. In the LCD condition, the aphasic subjects, but not the control subjects, displayed a signficant inhibitory effect. This effect indicates that they made significantly more errors in response to LCD targets preceded by an unrelated prime. 


\section{Discussion}

All three experimental groups were faster and more accurate in their responses to HCD targets than to LCD targets. This once again demonstrates the robustness of the dominance/frequency effect that has been observed throughout the series of studies reported here, and it indicates that sensitivity to word dominance/ frequency is maintained in older adults and in individuals with Broca's aphasia.

The two normal control groups performed similarly in their responses to HCD targets, although none of the individual priming effects were significant for the older control group. Nonetheless, the pattern of priming was similar for both groups, in that each displayed more facilitation for responses in the related condition than inhibition in the unrelated conditions. In contrast, the two control groups' responses to LCD targets were quite different. Unlike the young normal controls, the old normal controls displayed a large and significant $-128 \mathrm{~ms}$ inhibition effect, in addition to a significant +117 overall priming effect. Furthermore, their $-10 \mathrm{~ms}$ facilitation effect indicates that their mean RT was slightly slower in response to targets in the related condition than in response to targets in the neutral condition, although this effect was not statistically reliable. The young normal controls displayed a $+38 \mathrm{~ms}$ facilitation effect, and a $-27 \mathrm{~ms}$ inhibition effect with LCD targets, although, only their $66 \mathrm{~ms}$ overall priming effect was statistically reliable.

These diverging patterns of results for the LCD targets suggest an age effect. This result is surprising, since several investigators have reported no significant age differences for automatic or strategic priming effects (Madden, Pierce, \& Allen, 1993; 
Balota, Black, \& Cheney, 1992; Balota \& Duchek, 1988; Burke, White, \& Diaz, 1987). Howard, Shaw, \& Heisey, (1986) did report an age effect when they looked at the interaction of between age, priming, and SOA, however the results they obtained were quite different from those observed in Experiment 2. The young subjects displayed facilitation across the three SOA conditions examined $(150 \mathrm{~ms}, 450 \mathrm{~ms}$, and $1000 \mathrm{~ms})$, whereas the older adults only displayed facilitation in the longest SOA condition. None of the subjects displayed inhibition in any of the SOA conditions. Thus, unlike in the present study they did not see a large inhibitory effect for their older subjects, although they did suggest that the absence of facilitation in the shorter SOA conditions for the older normals may represent an age-related slowing in the spread of activation. Bowles and Poon (1988) also reported an age effect, that was due to a larger overall priming effect $(+135 \mathrm{~ms})$ in their older subjects compared to that observed in their younger group (+72 ms). But again, the individual patterns of facilitation and inhibition observed across their two groups did not conform to those observed in the present study. None of these studies examined semantic priming for high- versus low-dominance/ frequency targets across age groups. ${ }^{4}$ At a minimum, the results obtained for LCD targets across the two control groups in the present study suggest we need to know more about the interaction of priming, age, and word frequency/dominance. Apparently, Becker (1979) has published the only lexical priming study that specifically examined the interaction of priming and word frequency. He reported a larger overall priming effect for low-frequency words than for high-frequency words in a lexical decision task using a $1050 \mathrm{~ms}$ SOA. 
For the Broca's subjects, significant priming was observed for HCD targets in the reaction time data, but not the error data. In contrast, only the error data yielded a significant priming effect for $L C D$ targets. In response to $H C D$ targets, the Broca's aphasics performed quite differently from either of the two control groups. Their overall priming effect for HCD targets did not reach statistical significance, but suggests some sensitivity to the prime target relation, since RTs were $+95 \mathrm{~ms}$ faster in the related compared to the unrelated condition. In this respect, their data were similar to that of the two control groups. However, they displayed a large, and statistically reliable inhibition effect $(-146 \mathrm{~ms})$ indicating that responses were significantly slower to $\mathrm{HCD}$ targets preceded by an unrelated prime compared to those preceded by a neutral prime. Furthermore, their responses to HCD targets preceded by a related prime were also slower, by a mean of $51 \mathrm{~ms}$, than to those preceded by a neutral prime. Thus, responses to targets preceded by both related and unrelated primes were slower than responses to targets preceded by a neutral prime.

The Broca's subjects produced a high proportion of errors in response to LCD targets ( $31 \%)$, and the reaction time priming effects were negligible for this condition. However, their mean error rates to LCD targets, yielded a pattern of results similar to what was observed with the reaction time data for HCD targets. Again, the overall priming effect was not statistically reliable, but there was some indication that the Broca's subjects were sensitive to the prime target relation, since they made $20 \%$ fewer errors in response to LCD targets in the related priming condition compared to the unrelated priming condition. In addition, a large and statistically reliable inhibition 
effect was observed (-23.33) indicating that significantly more errors were made in response to targets preceded by an unrelated prime compared to those preceded by a neutral prime. Furthermore, responses were less accurate to targets in the related condition than in the neutral condition, although this - 3.33 facilitation effect was not significant.

It is of interest to note that the pattern of results observed for LCD targets in the older control groups' reaction time data was similar to that observed for both HCD and LCD targets in the aphasic group's data. This observation is even more intriguing since the same pattern was not observed in the young control subject's data. This introduces the possibility that the abnormal performance displayed by the Broca's aphasics is an exacerbation of a normal aging phenomenon. This possibility, as well as other interpretations of the Experiment 2 data will be addressed in the general discussion. However, before turning to that discussion, a final and important issue must be addressed. As noted previously, a small $\mathrm{n}$ always poses a threat to whether a measured behavior represents the norm observed in the larger population. This is an even more significant issue when exploring behavior in brain damaged individuals, wherein the hope of obtaining a homogeneous group of subjects poses an even greater challenge. To illustrate the magnitude of this problem, Table 27 presents a summary of individual subjects' priming effects (refer to Table 26 for group means). As may be seen, there is considerable variability in performance between subjects in each group. These data are presented, not with the intention of carrying out an intricate single case analysis of each subject. Nor are they intended to undermine the value of the data that has been 
Table 27. Individual subject RT and error priming effects, Experiment 2.

\begin{tabular}{cccc}
\multicolumn{2}{c}{ RT Priming Effects } & \multicolumn{2}{c}{ Error Priming Effects } \\
High & Low & High & Low \\
Dominance & Dominance & Dominance & Dominance
\end{tabular}

\begin{tabular}{|c|c|c|c|c|c|c|c|c|c|c|c|c|c|}
\hline & Facil & Inhib & Overall & Facil & Inhib & Overall & Facil & Inhib & Overall & Facil & Inhib & \multicolumn{2}{|l|}{ Qverall } \\
\hline \multicolumn{14}{|c|}{$\underline{\mathrm{AP}}$} \\
\hline 1 & -81 & -183 & +102 & -43 & -13 & -30 & .00 & -.10 & +.10 & .00 & -.30 & +.30 & \\
\hline 2 & -198 & -165 & -33 & +222 & +228 & -06 & .00 & -.20 & +.20 & .00 & -.30 & +.30 & \\
\hline 3 & +74 & +40 & +33 & +259 & +187 & +72 & .00 & .00 & .00 & .00 & .00 & .30 & \\
\hline 4 & +49 & -111 & +161 & +13 & -73 & +85 & -.00 & -.00 & .00 & -.30 & -.10 & -.20 & \\
\hline 5 & -11 & -232 & +221 & -282 & -66 & -215 & .00 & +.10 & -.10 & .00 & -.30 & +.40 & \\
\hline 6 & -141 & -226 & +84 & -161 & -308 & +147 & +.40 & +.10 & +.30 & .00 & -.40 & +.40 & \\
\hline \multicolumn{14}{|c|}{$\underline{O N}$} \\
\hline 1 & +66 & -83 & +149 & +22 & -188 & +210 & +.20 & +.20 & .00 & .00 & .00 & .00 & \\
\hline 2 & +18 & -19 & +37 & +87 & -35 & +122 & .00 & .00 & .00 & .00 & .00 & .00 & \\
\hline 3 & +124 & +62 & +61 & -196 & -130 & -66 & .00 & -.10 & +.10 & .00 & .00 & .00 & \\
\hline 4 & -38 & -02 & -36 & +27 & -188 & +215 & .00 & .00 & .00 & .00 & -.10 & +.10 & \\
\hline 5 & +12 & -13 & +25 & +50 & -80 & +130 & +.10 & +.10 & .00 & .00 & .00 & .00 & \\
\hline 6 & +95 & +17 & +77 & -50 & -145 & +94 & .00 & .00 & .00 & .00 & -.10 & +.10 & \\
\hline \multicolumn{14}{|c|}{$\underline{Y N}$} \\
\hline 1 & +159 & +02 & +157 & +129 & +73 & +55 & .00 & .00 & .00 & .00 & .00 & .00 & \\
\hline 2 & +04 & -83 & +87 & +95 & +132 & -36 & .00 & .00 & .00 & +.10 & +.10 & .00 & \\
\hline 3 & +50 & +19 & +32 & -68 & -289 & +221 & .00 & .00 & .00 & .00 & .00 & .00 & \\
\hline 4 & +43 & -77 & +120 & -26 & -117 & +90 & .00 & .00 & .00 & +.10 & -.20 & +.30 & \\
\hline 5 & +08 & -80 & +88 & +18 & -70 & +88 & .00 & -.10 & +.10 & .00 & .00 & .00 & $\vec{\omega}$ \\
\hline 6 & +52 & +20 & +33 & +82 & +107 & -25 & .00 & -.10 & +.10 & +.10 & -10 & +.30 & \\
\hline
\end{tabular}


gathered. Rather, they emphasize the need to exercise control when drawing conclusions based on measures as seemingly delicate as reaction time data, especially when small numbers of subjects are used. Nonetheless, it is encouraging to note that the RT data for the HCD targets and the error data for the LCD targets reflect a similar pattern of priming for all but one of the aphasic subjects (subject 3). Furthermore, the inhibition effect observed for LCD targets in the older control group data is seen in each of the individual older subject's data, although there is more variance in the degree to which they produced a facilitatory effect in the LCD condition. The younger control data illustrates the degree of variability that may be observed in supposedly "normal" data. Although the statistical analysis of these data yielded a significant facilitation effect without inhibition, as is normally reported in the literature and as was observed in Experiment 1, clearly each of the young normal controls did not produce this same pattern of effects. With these observations in mind, it is emphasized that the data obtained in Experiment 2, and indeed from any experiments using a particularly small n, should be interpreted with caution. Conversely, it is equally important to remember that trends observed in larger studies do not necessarily account for individual differences.

\section{End Notes}

1. Table 14 only includes examples of prime-target pairs with $H C D$, but of course, an equal number of LCD pairs will also be included.

2. Four of these replacements involved the same data file. The data for the first subject to complete the task in the 250 SOA condition with stimulus list version $\mathrm{C}$ were thrown out because of an overall error rate of $14 \%$. The data of the next three consecutive subjects who performed the task with the $250 \mathrm{C}$ stimulus file were discarded because their SD exceeded $200 \mathrm{~ms}$. Thus, although 8 subjects were replaced, only 4 data files were replaced. 
3. I wish to acknowledge the Louisiana State University Chapter of Sigma Xi, the Scientific Research Society, for a Graduate Student Research Grant-in Aid that assisted with subject payment.

4. Bowles and Poon (1988) did use a combination of high-, mid- and low-category dominance prime-target pairs, but unfortunately, they did not report the priming effects for the individual dominance conditions separately. 


\section{GENERAL DISCUSSION}

\section{The Neely Keefe Three Process Model of Priming}

The purpose of Experiment 1 was to determine if the three priming mechanisms described within the Neely and Keefe hybrid three process theory could be dissociated by examining the contribution of facilitatory and inhibitory effects to overall priming for HCD and LCD targets across multiple SOAs of increasing duration. Both a high relatedness proportion and a high nonword ratio were used to encourage strategic processing. Three predictions were formulated based on the Neely and Keefe model. First, a pattern of significant facilitation without significant inhibition, indicative of priming mediated by automatic spreading activation, was expected in the shortest SOA conditions. Second, significant inhibitory effects were expected in all but the shortest SOA conditions, as a sign that subjects were using strategies to complete the task. Third, significant inhibitory effects for high-dominance exemplars were expected to be observed before (i.e. with shorter SOAs) significant inhibition effects for lowdominance exemplars and before the appearance of significant nonword facilitation effects. The latter of these three predictions critically distinguishes the Neely-Keefe model from a two-process theory of priming. Facilitation and inhibition for high-, but not low-category dominance exemplars, and no nonword facilitation would be indicative of expectancy based priming. Facilitation and inhibition for both high- and low-category dominance exemplars, and a significant nonword facilitation effect would be indicative of semantic matching. 
The first of these predictions was supported by the data. Facilitation without a significant degree of inhibition was observed for both HCD and LCD targets in the two shortest SOA conditions. This result is consistent with the claim that priming in short SOA conditions is mediated by automatic spreading activation.

The second of these predictions was only partially met, as significant inhibitory effects were observed only in the 450 SOA condition. The observation of inhibitory effects in the 450 SOA condition, and the absence of inhibition in the shorter SOA conditions, are consistent with the claim that inhibitory effects signal the operation of a time-consuming strategic process. Therefore, despite the absence of inhibitory effects in the longer SOA conditions, the data do support a two-process (i.e., automatic vs. strategic) theory of priming. Possible explanations for the absence of inhibitory effects in the longer SOA conditions will be discussed below.

The third of these three predictions was not supported by the data. The inhibitory effects and overall priming effects observed for HCD and LCD targets in the 450 SOA condition were of similar magnitude. Furthermore, there was no SOA condition in which inhibitory effects were observed for HCD targets but not LCD targets. In addition, a nonword facilitation effect was not observed. The first implication of these results is that the present data do not allow one to clearly dissociate between the operation of an expectancy based priming mechanism and semantic matching as described within the Neely and Keefe model. The word target data suggest that subjects were using a semantic matching strategy in the 450 SOA condition, since Neely and Keefe's (1989) analysis of this strategy predicts equivalent priming for HCD 
and LCD targets. However, semantic matching is also argued to mediate nonword facilitation, which was not observed. As noted earlier, the unexpected pseudodominance effect for nonword targets complicates the interpretation of the nonword data, therefore the absence of a nonword facilitation effect is not necessarily problematic. A more significant problem is that the prediction consistent with expectancy based priming (i.e., inhibition for HCD targets but not LCD targets) was not observed. Despite this fact, there is some evidence that expectancy based priming may have been operating in the 350 SOA condition.

Inhibitory effects provide the clearest indication of strategic priming effects because inhibition of unrelated word targets cannot be explained by automatic spreading activation. Therefore, as originally formulated, the predictions regarding the expectancy and semantic matching mechanisms emphasized the presence or absence of inhibition. However, recall that Becker's (1980) analysis of expectancy based priming predicts that facilitation will be greater, and inhibition effects will be smaller, the smaller the expectancy set. It is reasonable to argue that in the 350 SOA condition subjects were beginning to consciously appreciate the prime, but only had time to develop a small expectancy set before the target appeared. If so, responses to HCD targets would have been facilitated since they would be quickly matched with members of the expectancy set. Responses to LCD targets would not be inhibited because a search through a small expectancy set would consume little time. Nor would they be facilitated since LCD targets would not likely be included in a small expectancy set. This possibility provides 
an explanation for the facilitation of HCD targets, but not LCD targets in the 350 SOA condition, that is otherwise problematic to interpret.

It is unlikely that the facilitation observed for HCD word-targets, but not LCD word-targets, in the 350 SOA condition was mediated by automatic spreading activation (ASA) for the following reasons. ASA can account for greater activation of strong associates than weak associates, if insufficient time is available for the spread of activation to reach the word nodes of weak associates. The fact that facilitation was observed for both HCD and LCD word targets in the two shortest SOA conditions indicates that the $150 \mathrm{~ms}$ and $250 \mathrm{~ms}$ SOAs provided sufficient time for activation to spread to the less strongly related LCD targets. Therefore, it seems improbable, that in the next longest SOA condition, ASA would have less of an impact on the processing of LCD targets. A more likely explanation is that spreading activation had already decayed, and that the facilitation observed for HCD was mediated by a different mechanism.

In summary, these results provide much weaker evidence for a dissociation between the two strategic priming mechanisms described by Neely and Keefe than was expected based on the predictions outlined above. This dissociation would have been more convincing had the inhibition effects associated with expectancy based priming been observed prior to (i.e., in a shorter SOA condition) the inhibition effects associated with semantic matching, and had nonword facilitation been observed in the longer SOA conditions. 
Despite these problems, facilitation without inhibition was observed in the two shortest SOA conditions, and a robust inhibitory effect was observed in the 450 SOA condition. Therefore the results do provide some empirical support for a two process theory of priming that involves both a fast-acting automatic component, and a timeconsuming strategic component. However, it is perplexing that significant inhibitory effects were observed in only the 450 SOA condition, and not in the longer SOA conditions.

One factor that needs to be considered to explain these results has to do with the appropriateness of using a neutral prime condition to determine the contribution of facilitation and inhibition to overall priming. A number of investigators have discussed the problem of finding a suitable baseline for measuring facilitatory and inhibitory effects, and it is possible that comparisons against a neutral prime either underestimate or overestimate these effects under certain experimental conditions (Antos, 1979; DeGroot et al., 1982; Jonides \& Mack, 1984; Neely, 1991). For example, it has been suggested that neutral primes may be processed more easily than word primes, simply by virtue of the fact that they are seen more frequently throughout the course of an experiment. If so, it is likely that they take less time to process than word primes. Thus, in short SOA conditions, responses to targets following word primes may be delayed relative to responses to targets following neutral primes, because subjects are still encoding the word prime when the target appears. This would result in an overestimation of inhibition, and an underestimation of facilitation. However, in the present experiment, an insignificant amount of inhibition was observed in the three 
shortest SOA conditions. Therefore, this potential problem with the neutral baseline cannot explain the obtained results. Furthermore, it cannot explain the significant degree of inhibition observed in the 450 SOA condition, since subjects had even more time to process word primes in this condition.

Another potential problem is that to serve as an effective baseline, neutral primes should have the same alerting qualities as do word primes. Yet, during the course of an experimental session, the same neutral prime is presented more frequently than any of the individual word primes, and it is quite possible that it does not maintain the same alerting value as do word primes. Neely (1991) suggests that this is more likely to be true in long SOA conditions, because subjects may be using the word primes to generate expectancies, and thereby maintaining a higher arousal to word primes. If the habituated neutral prime fails to provide the same level of arousal as do word primes, responses to targets following the neutral prime would be expected to be slower, resulting in an overestimation of facilitation and an underestimation of inhibition. Therefore, it is possible that habituation to the neutral prime contributed to the failure to observe significant levels of inhibition in the longest SOA conditions. Furthermore, there is reasonable evidence to suggest that subjects may have been less habituated to the neutral prime in the 450 SOA condition. Posner and Boise (1971) reported data suggesting that the alerting and encoding functions of a prime are optimal at about $500 \mathrm{~ms}$, as evidenced by faster reaction times to the subsequent target. Reaction times are slower with both shorter and longer time intervals. An examination of the mean reaction times reported in Table 5 reveals that responses to targets 
following a neutral prime were fastest in the 450 SOA condition. Therefore, it may be that in comparison to the other SOA conditions, both the alerting function and the encoding of the neutral prime were most complete in the 450 SOA condition. Based on experiments conducted in her laboratory, De Groot (1982) has also suggested that subjects may be optimally ready to respond to the target with an "SOA of $460 \mathrm{~ms}$ or thereabouts" (p. 360).

What remains puzzling, however, is why other investigators, who also used a neutral control condition, have reported significant inhibitory effects in longer SOA conditions. For the most part, the present data reflect a pattern of priming that Becker (1980) described as "facilitation dominant." That is, with few exceptions the facilitation effects were greater than the inhibitory effects. This result was unexpected, since investigations of priming conducted with category-exemplar stimuli and long SOA conditions ( $\geq 700 \mathrm{~ms}$ ) typically result in a pattern of inhibition dominance (Becker, 1980; DenHeyer et al., 1985; Favreau \& Segalowitz, 1983; Lorch et al., 1986; Neely, 1977, but see den Heyer et al., 1983; Neely et al., 1989). Careful review of the procedures used in previous studies revealed one critical difference with the procedures used in the current experiment that may account for this difference. In all but one case (DenHeyer et al., 1983), subjects were explicitly informed of the possible semantic relation between the prime words and target words. In the current experiment, subjects were not told about the prime-target relation. Interestingly, Den Heyer et al. (1983), who also did not inform their subjects of the prime target relation, observed a pattern of facilitation dominance in a high RP condition with a $1000 \mathrm{~ms}$ SOA. Den Heyer et al. 
(1985, pp 232) addressed the potential importance of this procedural differences in the following footnote:

It should be noted that the instructions to the subjects may be of critical importance. The experiment reported in this article represents a replication of an earlier effort with 34 subjects per group. In that procedure, subjects were given 10-20 practice trials and were not told of the nature of the stimuli and were not instructed about the different prime-target relationships. The results of that study failed to replicate the results of Experiment 1 and 2 reported by Becker (1980). In particular, the category data of the 1,000 ms SOA condition did not conform to an inhibition-dominant priming pattern, although it should also be noted that the error data did produce an inhibition-dominant pattern, suggesting that there may have been a speed-accuracy trade-off at work. It is possible that the change in instructions and the changing patterns of error rates are not independent.

These observations suggest that inhibitory effects signalling the use of strategic processing may not be observed unless subjects are explicitly encouraged to attend to the prime-target relation. Apparently, de Groot (1984) and De Groot et al. (1986) are the only investigators to report a significant degree of inhibition in a long SOA condition, when subjects were not explicitly informed of the prime target relation. Possibly they observed significant inhibitory effects because of the detailed feedback they provided subjects after every trial. In any event, the critical issue is that in addition to such factors as the composition of the stimulus list (e.g., a high or low relatedness proportion) and the prime-target stimulus onset asynchrony, the manner in which subjects are instructed seems to influence the degree to which they use conscious strategies during a semantic priming task. The fact that subjects were not explicitly alerted to the prime-target relation in the current experiment provides another possible explanation for the absence of significant inhibition in the longest SOA conditions. 
In general the results obtained in Experiment 1emphasize some of the problems inherent in analyzing the cost-benefit of reaction times in terms of facilitatory and inhibitory effects. The likelihood that subjects will display these effects in any given experiment appears to be vulnerable to many influences. As has already been noted, these include factors such as the composition of the stimulus list, the prime-target stimulus onset asynchrony, the selection of an appropriate neutral control condition, the instructions provided to subjects, and possibly the type of feedback subjects receive.

Another factor that may have influenced the obtained results is that the practice trials contained a different relatedness proportion (.50) and nonword ratio (.60) than the experimental trials $(\mathrm{RP}=.80, \mathrm{NWR}=.83)$. Following examples from the literature (e.g. Neely, Keefe, \& Ross, 1989), no attempt was made to equate these variables. However, Milberg et al., (1995) successfully demonstrated that the proportion cf related word-prime/word-target pairs presented immediately prior to experimental trials can serve as an "induction set", and influence subject responses during experimental trials. Immediately prior to the experimental trials, their subjects were presented with either a "high induction set", in which all real word pairs were semantically related, or a "low induction set", in which none of the word target pairs were semantically related. The presentation of these induction sets influenced the patterns of facilitation and inhibition that were observed in the first half of the experimental trials (44/88 total experimental pairs). Young normal subjects displayed facilitation and inhibition following the high induction set, but facilitation without significant inhibition following the low induction set. Broca's aphasics displayed facilitation and inhibition following the high induction 
set and inhibition without significant facilitation following the low induction set. These results confirm that the composition of a stimulus set presented to subjects prior to actual experimental trials can influence subjects' responses to experimental trials. Thus, one cannot entirely discount the possibility that the lower RP and NWR reflected in the composition of the practice materials that were presented to subjects prior to Experiment 1 reduced the impact of the high RP and high NWR contained in the experimental trials.

In summary, multiple methodological variations must be carefully considered when planning, and interpreting, priming studies, especially those using a neutral condition to examine facilitatory and inhibitory influences. Any results that are subject to contextual manipulations, whether facilitatory or inhibitory, must be considered strategic in nature, since one of the basic attributes of automatic activation is that it occurs independent of subject strategies or contextual factors other than the prime-target relationship. Unfortunately, the attempt to dissociate the effects of a prelexical expectancy mechanism and a postlexical semantic matching mechanism in Experiment 1 was not entirely successful, although there was some hint of a dissociation between the 350 and 450 SOA conditions.

Semantic Priming in Broca's Aphasia

The purpose of Experiment 2 was to determine if Broca's aphasics would display the same pattern of facilitation and inhibition effects as normal subjects in a pairwise lexical decision task presented with a $250 \mathrm{~ms} \mathrm{SOA}$ and a stimulus set designed to induce strategic processing. Their responses to $H C D$ targets clearly displayed a 
different pattern of results than that produced by both the young and old normal control subjects. Their responses to HCD targets in the related condition were $+95 \mathrm{~ms}$ faster than to those in the unrelated condition. However, unlike the control subjects, they produced a large, and statistically reliable inhibition effect $(-128 \mathrm{~ms})$, indicating that their responses were slower in response to $\mathrm{HCD}$ targets preceded by an unrelated prime compared to those preceded by a neutral prime. In addition, and unlike the control subjects, they displayed a $51 \mathrm{~ms}$ trend toward slower responses to HCD targets in the related condition compared to the neutral condition. The fact that responses to HCD targets were slower when preceded by either a related or unrelated prime than when preceded by a neutral prime suggests the possibility that the aphasic subjects processed word primes (i.e., category names) more slowly than the neutral prime, "blank".

A similar pattern of results was observed with the error data for LCD targets. Again, the overall priming effect was not statistically reliable, but there was some indication that the Broca's subjects were sensitive to the prime target relation, since they made $20 \%$ fewer errors in response to LCD targets in the related priming condition compared to the unrelated priming condition. In addition, unlike the young normal controls, they displayed a large and statistically reliable $-23.33 \%$ inhibition effect, indicating they made significantly more errors in response to targets preceded by an unrelated prime than to those preceded by a neutral prime. Furthermore, their responses were less accurate to targets in the related condition than in the neutral condition, although this - 3.33 facilitation effect was not significant. Once again, these data suggest that the aphasic subjects may have been able to process the neutral primes more 
easily than the word primes, since responses to targets preceded by word primes, either related or unrelated, were less accurate than those to targets preceded by the neutral prime.

The fact that the Broca's aphasics responses were slower and less accurate when preceded by word primes (whether related or unrelated) compared to the neutral prime suggests that their lexical processing of the prime words was slower than normal. However, the fact that the -51 facilitation effect for $\mathrm{HCD}$ targets and the $-3.33 \%$ facilitation effect for LCD targets were not statistically reliable, weakens the ability to make this claim with any confidence. On the other hand, the disparate findings for HCD versus $L C D$ targets provides more compelling evidence for slow lexical processing in the Broca's aphasics' data. It is likely that the large proportion of errors the aphasics made in response to LCD targets (31\%) was due to their frequent inability to respond to these targets within the time frame allowed. Unfortunately, the manner in which the DMASTR program records errors does not distinguish between errors resulting from incorrect responses and those resulting from responses exceeding the $4000 \mathrm{~ms}$ target duration cutoff. However, it seems reasonable to assume that the reason priming for LCD targets was observed in the error data, but not the RT data, was due to excessively slow responses to the LCD targets.

The old normal controls performed similarly to the young normal controls in their response to $H C D$ targets, although their +52 ms overall priming effect and $+46 \mathrm{~ms}$ facilitation effect did not reach statistical reliability. Their responses to LCD targets, however, were quite different from those of the young normals. In fact, the $-128 \mathrm{~ms}$ 
inhibition effect and the $-10 \mathrm{~ms}$ facilitation effect observed for LCD targets in the old normal data resembles the pattern of priming observed for $\mathrm{HCD}$ targets in the aphasic subjects' data. That is, their responses to targets following word primes, both related and unrelated, were slower than their responses to targets following neutral primes.

The large inhibitory effects produced by the older controls in response to LCD targets, and by the aphasic subjects in response to both HCD and LCD targets, suggests that these two subject groups were engaged in strategic processing, despite the fact that a short SOA condition was used to diminish this possibility. However, since both of these groups showed a tendency to respond more slowly to targets preceded by word primes, related or unrelated, than to targets preceded by neutral primes, raises a question about the adequacy of the neutral baseline, and hence the validity of the large inhibitory effects they displayed. Recall that in short SOA conditions, responses to targets following word primes may be delayed relative to responses to targets following neutral primes, if the neutral primes are processed more easily than word primes. If so, the result is an overestimation of inhibition and an underestimation of facilitation. Indeed, one cannot entirely discount the possibility that the facilitatory effects and inhibitory effects in these two groups were misestimated, at least to some degree. However, there is independent evidence in the older controls' data to suggest that the inhibitory effects displayed by these two groups were not entirely overestimated, if at all.

Automatic spreading activation predicts equal or greater facilitation of strong associates compared to weak associates, since it is assumed that ASA dissipates the farther it spreads from the priming word node. Since ASA cannot account for inhibitory 
effects, this means that not only facilitatory, but also overall priming effects, should be equal or greater for HCD targets compared to LCD targets. Therefore, if the priming effects observed in the older controls' data were mediated solely by automatic spreading activation, the overall priming effect for $\mathrm{HCD}$ targets should have been equal to or greater than the overall priming effect for LCD targets. In fact, their overall priming effect for LCD targets $(+117 \mathrm{~ms})$ was more than double that of the overall priming effect for HCD targets ( $+52 \mathrm{~ms})$. Since ASA cannot account for a larger overall priming effect for $\mathrm{LCD}$ targets than for $\mathrm{HCD}$ targets, these data suggest that at least part of the overall priming effect for LCD targets was mediated by a process other than ASA. It is worthy to note that in both Experiment 1 and Experiment 2 in the 250 SOA condition, the young normal subjects' overall priming effects were similar across the two dominance conditions, though slightly greater for the HCD targets.

This analysis suggests that the large inhibitory effect in the older controls' data was valid, which in turn makes it more reasonable to assume that the inhibitory effects for both HCD and LCD targets displayed by the aphasic subjects were also valid. Since ASA cannot account for inhibition, these inhibitory effects suggest that the subjects were engaged in some sort of strategic processing. If we accept Neely and Keefe's argument than an expectancy strategy would result in inhibition for HCD targets only, these data suggest that the older adults and the aphasic subjects were using a semantic matching strategy to aid their lexical decisions. This may seem counterintuitive however, since Neely and Keefe have argued that the semantic matching strategy does not operate at short SOA's. However, not all investigators limit the operation of post- 
lexical strategies to long SOA conditions. De Groot (1984, see also De Groot et al., 1986) has described a similar post-lexical matching strategy and argues that such a strategy would be "as effective at $240 \mathrm{~ms}$ SOAs as it is at the longer SOAs" (p. 275). In her view, any sort of post-lexical checking would operate relatively independent of the SOA, since the process is only effective after recognition of both the prime and target, but before the yes-no lexical decision is executed.

Despite the fact that a short SOA was used, there are a number of reasons to consider why the older control and aphasic subjects were able to engage in strategic processing even within this brief time interval. First, some investigators have argued that the pairwise lexical decision paradigm may induce subjects to use controlled processes if subjects notice that the prime-target pairs are presented together and use this information to aid their word/nonword decisions (Prather \& Swinney, 1988; Shelton \& Martin, 1992). Second, the particular stimulus set used in the present experiment was designed specifically to encourage the use of strategic processing by incorporating both a high relatedness proportion and a high nonword ratio. Third, investigations of sentence context effects on the recognition of single words indicate that any variable that delays or increases the difficulty of target word recognition causes an increase in inhibition and implicates the use of conscious, strategic processing -even in experimental conditions that otherwise yield a pattern of facilitation without significant inhibition. For example, 1) younger readers and poor readers display larger inhibition effects than do adults and more skilled readers (e.g., Schvaneveldt, Ackerman, \& Semlear, 1977; Stanovich and West, 1978) , 2) greater inhibitory effects 
have been reported with "difficult" words compared to "easy" words, as distinguished by word frequency and length (Stanovich \& West, 1981), and 3) greater inhibitory effects are observed when the visual quality of the target stimuli is degraded (e.g., Forster, 1976). Stimulus degradation has also been observed to increase contextual effects in single-word priming studies as well (e.g. Becker \& Killian, 1977).

The results of Experiment 2 suggest that although under normal circumstances subjects will demonstrate evidence of automatic priming effects in a short SOA condition in a pairwise lexical decision, strategic processing can be elicited in these same conditions. The data suggest that under conditions wherein the stimuli cannot be processed automatically, either because of the processing limitations of the subjects, or the difficulty of the stimuli, that subjects will appeal to the use of strategic processing to complete the task, even in a short SOA condition. This was evidenced in the data of the old normal controls who displayed inhibition for the LCD targets, but not the HCD targets, and in the data of the aphasic subjects who displayed significant inhibition in both dominance conditions. A further test of this hypothesis would be to examine the impact of stimulus degradation with young normal subjects using the same stimulus materials and $250 \mathrm{~ms} \mathrm{SOA}$ condition used in Experiment 2. Degrading the visual quality of the stimuli would increase the likelihood that even young normal subjects would be unable to process the targets automatically. If so, the expected result would be that they would display evidence of strategic processing similar to what was observed in the data of the aphasics and older controls in Experiment 2. Although previous studies have demonstrated increased contextual processing with stimulus 
degradation, this apparently has not been examined with SOAs shorter than $750 \mathrm{~ms}$. The critical question is, will young normal subjects display strategic effects in a short SOA condition, if sufficiently challenged?

In summary, it seems reasonable to conclude that automatic lexical activation was reduced or slowed in both the older controls, as well as in the Broca's subjects, though not to the same degree. When challenged by their inability to process the stimuli automatically, both groups appealed to the use of conscious strategies to complete the task as normally as possible. In this sense, their strategic processing may be considered compensatory. In the case of the old normals, the resort to compensatory strategic processing was only necessary when challenged by the more difficult LCD targets. We can assume that the LCD targets were more difficult to process than the HCD targets given the persistent dominance effect that has been observed throughout the series of studies reported here.

Perhaps the most important contribution these data offer is that strategic priming effects can be elicited in a pairwise lexical decision task presented with a $250 \mathrm{~ms}$ SOA. Under normal circumstances, this task yields a priming pattern of facilitation without inhibition in short SOA conditions, indicative of automatic processing, such as was observed in the young normals' data in Experiment 1 and Experiment 2 and is typically reported in the literature. Given circumstances where subjects cannot adequately process the stimuli automatically however, it appears that they can appeal to the use of strategic processing, even at this short SOA. In the present experiment, this was 
demonstrated by including two groups of subjects in whom it is reasonable to assume that automatic lexical processing may be slowed.

Another important contribution of the present results is the observation that an overall priming effect can indeed mask abnormal priming, even in a short SOA condition. These results provide support for the argument made by Shelton and Martin (1992) and Prather et al. (1992, 1997), that the pairwise lexical decision task is inadequate for making claims about intact automatic activation, unless some sort of internal check such as a neutral priming condition is used. The inclusion of a neutral control condition in the present experiment illustrated how some individuals may resort to the use of strategic processing, even in conditions wherein subjects normally do not, if they are sufficiently challenged. The fact that the older control subjects and the Broca's aphasics displayed evidence of strategic processing, whereas the younger control subjects did not, suggests that their automatic processing capacities were inadequate to meet the demands of the task.

The apparent age effect that was observed in the present experiment suggests that spreading activation may be slowed in the normal elderly, as suggested by Howard et al. (1986). Such a hypothesis is congruent with the observation that information processing rates decrease with normal aging (Cerella \& Hale, 1994). It is likely that the failure to observe an age by priming interaction in many previous experiments was due to the fact that the task demands were not great enough to reveal the older subjects' deficit. In the present experiment, the age effect was revealed due to the inclusion of the more difficult to process LCD targets. 
The fact that the Broca's aphasics performed very similarly to the old normal controls, although they had difficulty processing both the HCD and LCD targets, suggests that their reduced lexical activation may be an exacerbation of a normal aging process. However, such a suggestion must be considered somewhat speculative based on the limited amount of data presented here, since similar behavior patterns do not necessarily imply similar underlying impairments. For example, Swinney, Zurif, and Nicol (1989) have suggested that slowed lexical slowing processing in Broca's aphasia may be viewed as a problem internal to the language module, and such an account is not congruent with the generalized cognitive slowing associated with aging (Cerella and Hale, 1994). Nonetheless, whether their underlying problems are the same or not, it appears that both groups had difficulty processing some of the stimuli automatically and attempted to compensate for their deficits in similar ways.

Prather and her colleagues $(1992,1997)$ have asserted that automatic activation is intact, but delayed in Broca's aphasics. Recall that with the list priming paradigm, a task that is not conducive to the use of strategies, Broca's aphasics displayed priming with a much longer ISI $(1500 \mathrm{~ms})$ than normals. Thus, they suggested that Broca's aphasics may activate lexical information automatically, but in a slower than normal fashion. The data obtained in the present experiment cannot address this issue since the priming task was presented with a single, short SOA. The present data offer no evidence that automatic activation in Broca's aphasics is intact. One way to further examine Prather et al.'s claim would be to attempt to replicate the results they obtained 
with the list priming paradigm, using the same aphasic subjects that participated in Experiment 2.

Milberg et al. (1995) have argued that the lexical processing deficit in Broca's aphasia reflects a reduction in the level of automatic activation of lexical information. Furthermore, they have suggested that the consequence of reduced levels of activation within a semantic network would be less consistent, smaller, or delayed priming effects, except in conditions that are conducive to the use of strategies. In turn, they have also suggested that an overreliance on the use of strategies by Broca's aphasics may have masked their impairment in automatic processing. The implication of this suggestion is that prior reports of intact priming, even in short SOA/ISI conditions, could have actually reflected Broca's aphasics ability to use strategic processes, not intact automatic processing. The data reported in the present experiment support this hypothesis. Despite the fact that the Broca's aphasics displayed evidence (though not statistically significant) of an overall priming effect, they displayed no hint of facilitation as would be expected with intact automatic processing. In contrast, they displayed a large and statistically significant inhibitory effect for both the HCD and LCD targets, implicating their use of compensatory strategies. The fact that these inhibitory effects were obtained with a $250 \mathrm{~ms}$ SOA condition, in which young normal controls displayed the typical pattern of facilitation without inhibition associated with automatic processing, directly challenges the claim made by Hagoort (1997) and Tyler et al. that automatic processing is intact in Broca's aphasia. 


\section{Summary and Conclusions}

The results of both Experiment 1 and Experiment 2 support the claim that under normal circumstances, the presentation of prime target pairs with a short SOA in a pairwise lexical decision task serves primarily as a measure of automatic lexical processing. Facilitation without inhibition was observed for both HCD and LCD targets in 150 and $250 \mathrm{~ms}$ SOA conditions, despite the use of stimulus set designed to induce the use of conscious strategies. The attempt to dissociate the effects of a prelexical expectancy mechanism and a postlexical semantic matching mechanism in Experiment 1 was not successful, although there was a hint of a dissociation in the 350 and 450 SOA conditions. In the 350 SOA condition, facilitation without inhibition for the HCD targets alone was interpreted as a possible sign that subjects were beginning to use an expectancy strategy to perform the task. However, a significant inhibitory effect for HCD targets, which would have provided a clearer indication that subjects were using an expectancy strategy, was not observed. In the 450 SOA condition, a robust inhibitory effect was observed for both HCD and LCD targets, suggesting that subjects may have been using a semantic matching strategy in this condition. However, the inhibitory effects expected in the longer SOA conditions were not observed, nor was the expected nonword facilitation effect. The fact that a large inhibitory effect was observed in the 450 condition, but not the later SOA conditions, was possibly due to subjects' devoting maximal attention to the neutral prime when prime-targets were presented with this time interval. Interpretation of the nonword data was complicated by the intrusion of an unexpected "pseudodominance effect" that is difficult to explain. 
Possibly subjects were attending to sublexical cues such as familiar letter sequences that biased their decisions to the nonwords. In any event, the most puzzling aspect of the Experiment 1 data was the failure to observe inhibition to unrelated word targets in the longer SOA conditions. A number of factors that may have contributed to this finding were enumerated, but it seems the most likely explanation is that subjects were not informed a priori of the potential relationship between primes and targets.

The series of pilot studies served to illustrate the difficulty in replicating standard priming effects with a within subjects design and a small number of subjects. Despite careful attempts to counterbalance stimuli and space experimental sessions to minimize the potential effects of repetition and practice in Pilot 1 and Pilot 2.B., significant priming effects were either reduced or extinguished with these within subjects designs. An additional factor that likely limited the ability to replicate the results of Experiment 1 in these studies was the use of a smaller number of subjects. The results of Pilot 2.A, which also used a small $\mathrm{n}$, but incorporated a between-subjects design, only yielded a partial replication of the results of Experiment 1.

One interesting observation is that despite their weaknesses, each of the pilot studies replicated the dominance/frequency effect that was observed in Experiment 1. Furthermore this effect was observed in all three subject groups in Experiment 2. Thus sensitivity to this lexical feature appears quite strong, since it is not easily diminished. As noted in earlier discussion it cannot be determined whether this effect resulted from sensitivity to category dominance or word frequency since these two factors were confounded in the stimuli used for these experiments. Though the existence of a 
frequency effect is one of the most well established findings in the lexical priming literature, it is unclear whether a pure "dominance effect" exists. One way to examine this issue would be to examine priming for high- and low-category dominance exemplars that are carefully equated in terms of word frequency. Thus far the results of a few studies that have attempted this are ambiguous.

With regard to semantic priming in Broca's aphasia, the results presented here best support the hypothesis presented by Milberg et al. (1995), who have argued that Broca's aphasics are unable to processing lexical information normally due to reduced levels of automatic activation. Furthermore, they support Milberg et al.'s claim that Broca's aphasics display an overreliance on the use of "heuristic strategies" that has previously masked their automatic processing deficit. In the present study, there was a suggestion of an overall priming effect in the aphasic's data that was similar to that observed in the young normals. However, the pattern of facilitation and inhibition they displayed was quite different from that displayed by the young normal subjects. It is interesting to note that despite the potential problems surrounding the use of a neutral baseline, it was the inclusion of the neutral priming condition in Experiment 2 that revealed the differences between the three groups of subjects.

Whether the automatic processing deficit observed in Broca's aphasics is best characterized in terms of speed of processing as suggested by Prather et al. (1992, 1997), or strength of lexical activation as suggested by Milberg et al. (1995) cannot be determined from the present data. However, in many cases it seems the consequences would be the same since according to Milberg et al., one effect of an impairment at the 
level of lexical activation would be a delay in the time course of lexical activation. In either case, the fact that the Broca's aphasics' impairment seems to involve automatic processing routines implies a reduction in their ability to normally decode lexical information in real time. Several investigators have suggested that such an impairment underlies Broca's aphasics inability to comprehend certain complex syntactic structures that require re-activation of lexical information at critical points in time during sentence processing (e.g., Swinney, \& Zurif, 1994, 1995).

The similarity between the results obtained for the Broca's aphasics and those obtained for the older controls suggests that subjects will resort to the use of strategic processing whenever their ability to process stimuli automatically is limited. Additional support for this view comes from those studies reporting greater contextual effects in slow and younger readers, and with degraded and difficult stimuli. These observations support the view of strategic processing as a compensatory measure that subjects adopt whenever they are unable to meet the normal demands of the priming task. What is less clear however, is to what degree subjects are likely to appeal to the use of conscious strategies under normal circumstances. As noted previously, it seems likely that the reason few strategic (i.e. inhibitory) effects were observed in Experiment 1, was because subjects were not explicitly informed of the prime-target relation. Thus, it appears that the primary reasons that subjects are likely to engage in strategic processing are 1) when their automatic processing capacity is inadequate to meet the specific demands of the task - due to subjects' own limitations, or, due to the quality or difficulty of the stimuli used, and 2) when explicitly encouraged to do so. In other words, many subjects may 
not resort to the use of strategies unless they are told to do so, or when they cannot complete the task otherwise. In turn, this suggests that whenever possible, access to the lexicon may proceed automatically, and independent of contextual influences other than those internal to the lexicon. 


\section{REFERENCES}

Antos, S. J. (1979). Processing facilitation in a lexical decision task. Journal of Experimental Psychology: Human Perception and Performance 5 (3) 527-545.

Balota, D. A. (1983). Automatic semantic activation and episodic memory encoding. Journal of verbal learning and verbal behavior, 22, 88-104.

Balota, D. A., Black, S. R., \& Cheney, M. (1992). Automatic and attentional priming in young and older adults: Reevaluation of the two-process model. Journal of Experimental Psychology: Human Perception, and Performance, 18 (2), 485-502.

Balota, D. A., \& Duchek, J. M. (1988). Age-related differences in lexical access, spreading activtion, and simple pronunciation. Psychology and Aging. 33 84-93.

Balota, D. A., \& Lorch, R. (1986). Depth of automatic spreading activation: Mediated priming effects in pronunciation but not in lexical decision. Journal of Experimental Psychology: Learning, Memory, \& Cognition, 12, 336-345.

Battig, W. F., \& Montague, W. E. (1969). Category norms for verbal items in 56 categories. Journal of Experimental Psychology, Monograph, 80 (3, Pt. 2), 1-45.

Becker, C. A. (1980). Semantic context in visual word recognition: An analysis of semantic strategies. Memory \& Cognition, 8, 493-512.

Becker, C. A. (1985). What do we really know about semantic context effects during reading? In D. Besner, T. G. Waller, \& E. M. MacKinnon (Eds.), Reading research: Advances in theory and practice, Vol. 5 (pp. 125-166). Toronto: Academic Press.

Becker, C. A., \& Killion, T. H. (1977). Interaction of visual and cognitive effects in word recognition. Journal of Experimental Psychology: Human Perception and Performance, 3 389-401.

Burke, D. M., White, H., \& Diaz, D. L. (1984). Semantic priming during sentence processing by young and older adults. Developmental Psychology, 20, 903-910.

Bushell, C. M. (1996). Dissociated identity and semantic priming in Broca's aphasia: How controlled processing produces inhibitory semantic priming. Brain and Language, 55, 264-288.

Blumstein, S. E., Milberg, W., \& Shrier, R. (1982) Semantic processing in aphasia: Evidence from an auditory lexical decision task. Brain and Language, 17, 301-315. 
Bowles, N. L., \& Poon, L. W. (1988). Age and context effects in lexical decision: An age by context interaction. Experimental Aging Research, 14 (4) 201-205

Cerella, J., \& Hale, S. (1994) The rise and fall in information-processing rates over the life span. Acta Psychologica, 86, 109-197.

Chenery, H. J., Ingram, J. C. L., \& Murdoch, B. E. (1990) Automatic and volitional semantic processing in aphasia. Brain and Language, 38, 215-232.

De Groot, A. M. B., Thomassen, A. J. W. M., \& Hudson, P. T. W. (1982). Associative facilitation of word recognition as measured from a neutral prime. Memory \& Cognition, 10 (4) 358-370.

de Groot, A. M. B. (1984). Primed lexical decision: Combined effects of the proportion of related prime-target pairs and the stimulus-onset asynchrony of prime and target. Quarterly Journal of Experimental Psychology, 36A, 253-280.

de Groot, A. M. B. (1985). Word-context effects in word naming and lexical decision. Quarterly Journal of Experimental Psychology, 37A. 281-297.

de Groot, A. M. B., Thomassen, A. J. W. M., \& Hudson, P. T. W. (1986). Primedlexical decision: The effect of varying the stimulus-onset asynchrony of prime and target. Acta Psychologica, 61, 17-36.

den Heyer, K. (1985). On the nature of the proportion effect in semantic priming. Acta Psychologica, 60, 25-38.

den Heyer, K., Briand, K., \& Dannenbring, G. L. (1983). Strategic factors in a lexicaldecision task: Evidence for automatic and attention-driven processes. Memory \& Cognition, 11 (4) 374-381.

den Heyer, K., Briand, K., \& Smith, L. (1985). Automatic and strategic effects in semantic priming: An examination of Becker's verification model. Memory \& Cognition, 13 (3) 228-232.

den Heyer, K., Goring, A., \& Dannenbring, G. L. (1985) Semantic priming and word repetition: The two effects are additive. Journal of Memory and Language, 24, 699-716.

Durgunoglu, A. Y. (1986). A comparison of the two versions of the interactivecompensatory model of reading. Unpublished doctoral dissertation. Purdue University. 
Durgunoglu, A. Y. (1988). Repetition, semantic priming, and stimulus quality: Implications for the interactive-compensatory reading model. Journal of Experimental Pschology 14 (4), 590-603.

Favreau, M., \& Segalowitz, N. S. (1983). Automatic and controlled processes in the first- and second-language reading of fluent bilinguals. Memory \& Cognition, $11,565-$ 574.

Fischler, I. (1977). Semantic facilitation without association in a lexical decision task. Memory \& Cognition, 5 (3), 335-339.

Forster, K. I. (1976). Accessing the mental lexicon. In R. J. Wales \& E. Walker (Eds.), New Approaches to Language Mechanisms. pp. 259-287. Amserdam: North-Holland.

Forster, K. I. (1979). Levels of processing and the structure of the language processor. In E. C. T. Walker \& W. E. Cooper (Eds.), Sentence processing: Psycholinguistic studies presented to Merrill Garrett. (27-84). Hillsdale, NJ: Lawrence Erlbaum Publishers

Forster, K. I. (1981). Priming and the effects of sentence and lexical contexts on naming time: Evidence for autonomous lexical processing. Quarterly Journal of Experimental Psychology, 33A, 465-495.

Goodglass, H., \& Baker, E. (1976). Semantic field, naming, and auditory comprehension in aphasia. Brain and Language, 3, 359-374.

Grober, E., Perecman, E., Kelar, L., \& Brown, J. (1980) Lexical knowledge in anterior and posterior aphasics. Brain and Language, $10,318-330$.

Hagoort, P. (1993) Impairments of lexical-semantic processing in aphasia: Evidence from the processing of lexical ambiguities. Brain and Language, 38, 215-232.

Hagoort, P. (1997) Semantic priming in Broca's aphasics at a short SOA: No support for an automatic deficit. Brain and Language, 56, 287-300.

Howard, D. V., Shaw, R. J., \& Heisey, J. G. (1986). Aging and the time couse of semantic activation. Journal of Gerontology, 41 195-203.

Huttenlocher, J., \& Kubicek, L. (1983). The source of relatedness effects on naming latency. Journal of Experimental Psychology: Learning, Memory, \& Cognition,2, 486496. 
Katz, W. F. (1988). An investigation of lexical ambiguity in Broca's aphasics using an auditory lexical priming technique. Neuropsychologia, 26 (5), 747-752.

Keefe, D. E., \& Neely, J. H. (1990) Semantic priming in the pronunciation task: The role of prospective prime-generated expectancies. Memory \& Cognition, 18, 289-298.

Kertesz, A. (1982). Western Aphasia Battery. NY: Grune \& Stratton.

Koriat, A. (1981). Semantic facilitation in lexical decision as a function of prime-target association. Memory \& Cognition, 9 , 587-598.

Kucera, H., \& Francis, W. N. (1967). Computational analysis of present day American English. Providence, RI: Brown University Press.

Lorch, R. F., Balota, D. A., \& Stamm, E. G. (1986) Locus of inhibition effects in the priming of lexical decisions: pre-or postlexical access? Memory \& Cognition. 14 (2), 95-103.

Maxwell, S. E. (1990). Designing experiment and analyzing data: A model comparison perspective. Wadworth Publishing Company: Belmont, CA.

Meyer, D. E., \& Schvaneveldt, R. W. (1971). Facilitation in recognizing pairs of words: Evidence of a dependence between retrieval operations. Joumal of Experimental Psychology, 90 (2), 227-234.

Milberg, W., \& Blumstein, S. E. (1981) Lexical decision and aphasia: Evidence for semantic processing. Brain and Language, 14, 371-385.

Milberg, W., Blumstein, S. E., \& Dworetzky, B. (1987) Processing of lexical ambiguities in aphasia. Brain and Language, 31, 138-150.

Milberg, W., Blumstein, S., \& Dworetzky, B. (1988) Phonological processing and lexical access in aphasia. Brain and Language, 34, 279-293.

Milberg, W., Blumstein, S. E., Katz, D., Gershberg, F., \& Brown, T. (1995). Semantic facilitation effects of time and expectancy Journal of Cognitive Neuroscience, 7 (1), 3350 .

Neely, J. H. (1976). Semantic priming and retrieval from lexical memory: Evidence for facilitatory and inhibitory processes. Memory \& Cognition, 4 (5), 648-654. 
Neely, J. H. (1977). Semantic priming and retrieval from lexical memory: Roles of inhibitionless spreading activation and limited-capacity attention. Journal of Experimental Psychology: General, 106 (3), 226-254.

Neely, J. H. (1991). Semantic priming effects in visual word recognition: a selective review of current findings and theories. In D. Besner \& G. W. Humphreys (Eds.), Basic processes in reading: Visual word recognition. (pp. 264-336). Hillsdale, NJ: Lawrence Erlbaum Associates, Publishers.

Neely, J. H., \& Keefe, D. E. (1989). Semantic context effects on visual word processing: A hybrid prospective-retrospective processing theory. In G. H. Bower (Ed.), The psychology of learning and motivation. (pp. 207-248). NY: Academic Press.

Neely, J. H., Keefe, D. E., \& Ross, K. L. (1989). Semantic priming in the lexical decision task: Roles of prospective prime-generated expectancies and retrospective semantic matching. Joumal of Experimental Psychology: Learning, Memory, and Cognition, 15 (6) 1003-1019.

Ostrin, R. K., \& Tyler, L. K. (1993) Automatic access to lexical semantics in aphasia: Evidence from semantic and associative priming. Brain and Language, 45, 147-159.

Posner, M. I., \& Snyder, C. R. R. (1975a). Attention and cognitive control. In R. L. Solso (Ed.) Information processing and cognition: The Loyola Symposium (55-85). NY: John Wiley \& Sons.

Posner, M. I., \& Snyder, C. R. R. (1975a). Facilitation and inhibition in the processing of signals. In P. M. A. Rabbit \& Dornic, S. (Eds.) Attention and Performance, V. NY: Academic Press.

Prather, P. A., \& Swinney, D. A. (1988) Lexical processing and ambiguity resolution: An autonomous process in an interactive box. In S. L. Small, G. W.Cottrell, \& M. K. Tanenhaus (Eds.), Lexical ambiguity resolution: Perspectives from psycholinguisitcs, neuropsychology, and artifical intelligence. (pp. 289-310). San Mateo, CA: Morgan Kaufmann Publishers, Inc.

Prather, P., Zurif, E., Stern, C., \& Rosen, T. J. (1992) Slowed lexical access in nonfluent aphasia: A case study. Brain and Language, 43, 336-348.

Prather, P. A., Zurif, E., Love, T., \& Brownell, H. (1997). Speed of Lexical Activation in nonfluent Broca's aphasia and fluent Wernicke's aphasia. Brain and Language, 59. $391-411$. 
Pring, L., \& Snowling, M. (1986). Developmental changes in word recognition: An information processing account. Quarterly Joumal of Experimental Psychology 38A, 395-418.

Rubenstein, H., Garfield, L., \& Millikan, J. A. (1970). Homographic entries in the internal lexicon. Joumal of Verbal Learning and Verbal Behavior, 9, 487-494.

Scarborough, D. L., Cortese, C., \& Hollis, S. S. (1977). Frequency and repetition effects in lexical memory. Joumal of Experimental Psychology: Human, Perception, and Performance, 3 (1), 1-17.

Schvaneveldt, R., Acker man, B. P., \& Semlear, T. (1977). The effect of semantic context on children's word recognition. Child Development, 48 612-616.

Seidenberg, M. S., Waters, G. S., Sanders, M., \& Langer, P. (1984). Pre- and postlexical loci of contextual effects on word recognition. Memory \& Cognition, 12, 315-328.

Shelton, J. R., \& Martin, R. C. (1992) How semantic is automatic semantic priming? Journal of Experimental Psychology: Learning, Memory, and Cognition, 18 (6), 1191 1210.

Stanovich, K. E., \& West, R. F. (1981). The effect of sentence context on ongoing word recognition: tests of a two-process theory. Journal of Experimental Psychology: Human Perception and Performance, 7 (3) 658-672.

Stanovich, K. E., \& West, R. F. (1983). On priming in a sentence context. Journal of Experimental Psychology: General, 112, 1-36.

Swinney, D., \& Zurif, E. (1995). Syntactic processing in aphasia. Brain and Language, 50, 225-239.

Swinney, D., Zurif, E., \& Nicol, J. (1989) The effects of focal brain damage on sentence processing: an examination of the neurological organization of a mental module. Journal of Cognitive Neuroscience, 1 (1), 25-37.

Tweedy, J. R., Lapinski, R. H., \& Schvaneveldt, R. W. (1977) Semantic-context effects on word recognition: Influence of varying the proportion of items presented in an appropriate context. Memory \& Cognition, 5 (1), 84-89.

Tweedy, J. R., \& Lapinski, R. H. (1981). Facilitating word recognition: evidence for strategic and automatic factors. Quarterly Journal of Experimental Psychology, 33A. 51-59. 
Tyler, L. K., Ostrin, R. K, Cooke, M., \& Moss, H. E. (1995). Automatic access of lexical information in Broca's aphasics: Against the automaticity hypothesis. Brain and language, $48,131-162$.

West, R. F., \& Stanovich, K. E. (1978). Source of inhibition in experiments on the effect of sentence context on word recognition. Journal of Experimental Psychology, Learning, Memory, and Cognition, 8 385-399.

Whaley, C. P. (1978). Word-nonword classification time. Journal of Verbal Leaming and Verbal Behavior, 17, 143-154.

Wilding, J. (1986). Joint effects of semantic priming and repetition in a lexical decision task: Implications for a model of lexical access. Quarterly Journal of Experimental Psychology, 38A, 213-228.

Zurif, E. B., Caramazza, A., Myerson, R., \& Galvin, J. (1974) Semantic feature representations for normal and aphasic language. Brain and Language, 1, 167-187. 
Stimuli selected for each category. List Freq refers to the frequency with which category exemplars were named for each category (Battig \& Montague, 1967). Wd Freq refers to frequency of occurrence (Kucera \& Francis, 1967). L refers to word length. NW refers to nonword. For each category, the first five items listed are high category exemplars, and the last five are low category exemplars.

\begin{tabular}{llrrrll}
\hline \multirow{5}{*}{ Category } & & List & Wd & & & \\
\hline \multirow{4}{*}{ Cloth } & Exemplar & Freq & Freq & L & NW Source & NW \\
& cotton & 404 & 36 & 6 & donkey & lonkel \\
& wool & 347 & 10 & 4 & lamp & bant \\
& silk & 292 & 13 & 4 & bolt & lolt \\
& rayon & 225 & 00 & 5 & brass & drass \\
& nylen & 210 & 01 & 5 & cider & ciler \\
& madras & 32 & 00 & 6 & square & squapa \\
& felt & 11 & 01 & 4 & golf & molf \\
& lace & 10 & 08 & 4 & warm & carm \\
& tweed & 14 & 05 & 5 & mouse & touse \\
& crepe & 11 & 01 & 5 & spear & speap \\
& dog & 426 & 147 & 3 & rug & rus \\
& cat & 412 & 42 & 3 & gas & lar \\
& horse & 348 & 203 & 5 & nurse & nurne \\
& cow & 284 & 46 & 3 & fir & fid \\
& lion & 225 & 26 & 4 & ship & stip \\
& fox & 44 & 11 & 3 & dry & bry \\
& elk & 11 & 02 & 3 & cap & pac \\
& moose & 33 & 00 & 5 & lance & cance \\
& ape & 4 & 03 & 3 & rib & bir \\
& bull & 40 & 16 & 4 & jeep & meep \\
& blue & 438 & 126 & 4 & mule & wule \\
& red & 435 & 169 & 3 & owl & wol \\
& green & 431 & 85 & 5 & couch & bouch \\
yellow & 387 & 52 & 6 & burlap & furlap \\
& black & 314 & 165 & 5 & frost & trosh \\
& & & & & &
\end{tabular}




\begin{tabular}{|c|c|c|c|c|c|c|}
\hline & aqua & 31 & 00 & 4 & harp & barp \\
\hline & $\tan$ & 42 & 00 & 3 & bow & fow \\
\hline & mauve & 13 & 00 & 5 & shift & shist \\
\hline & indigo & 47 & 01 & 6 & celery & cenery \\
\hline & navy & 09 & 48 & 4 & pony & dony \\
\hline \multirow[t]{10}{*}{ Body } & legs & 402 & 126 & 4 & lamb & pamb \\
\hline & arms & 398 & 217 & 4 & pink & pind \\
\hline & head & 308 & 449 & 4 & duck & gock \\
\hline & eye & 303 & 524 & 3 & fog & pol \\
\hline & foot & 295 & 361 & 4 & pipe & bipe \\
\hline & back & 37 & 190 & 4 & robe & dobe \\
\hline & skin & 10 & 53 & 4 & tuba & fube \\
\hline & chin & 10 & 25 & 4 & wolf & nolf \\
\hline & hip & 26 & 17 & 3 & fan & lan \\
\hline & bone & 20 & 53 & 4 & nuts & luts \\
\hline \multirow[t]{10}{*}{ Fruit } & apple & 429 & 15 & 5 & llama & barma \\
\hline & pear & 326 & 08 & 4 & dove & bove \\
\hline & banana & 283 & 05 & 6 & canary & panary \\
\hline & grape & 247 & 10 & 5 & brown & trown \\
\hline & cherry & 183 & 06 & 6 & kidney & gidnek \\
\hline & prune & 44 & 02 & 5 & scarf & scarg \\
\hline & lime & 67 & 13 & 4 & frog & frow \\
\hline & raisin & 16 & 01 & 6 & willow & rillow \\
\hline & berry & 11 & 05 & 5 & banjo & fanjo \\
\hline & papaya & 08 & 00 & 6 & calico & baliso \\
\hline \multirow[t]{10}{*}{ Tool } & hammer & 431 & 06 & 6 & rabbit & tabbit \\
\hline & saw & 394 & 08 & 3 & hot & hol \\
\hline & nail & 248 & 20 & 4 & gold & gorf \\
\hline & level & 168 & 265 & 5 & raven & ragen \\
\hline & plane & 147 & 138 & 5 & stove & stope \\
\hline & sander & 17 & 01 & 6 & pickle & bickle \\
\hline & awl & 18 & 00 & 3 & cub & cug \\
\hline & file & 26 & 59 & 4 & lung & lunt \\
\hline & plumb & 16 & 01 & 5 & skirt & skirb \\
\hline & wedge & 15 & 04 & 5 & brain & braig \\
\hline \multirow[t]{3}{*}{ Vehicle } & car & 407 & 393 & 3 & pig & mib \\
\hline & bus & 300 & 42 & 3 & emu & mue \\
\hline & airplane & 280 & 21 & 8 & lacrosse & tacrosse \\
\hline
\end{tabular}

Reproduced with permission of the copyright owner. Further reproduction prohibited without permission. 


\begin{tabular}{|c|c|c|c|c|c|c|}
\hline & train & 257 & 86 & 5 & clerk & clers \\
\hline & truck & 223 & 80 & 5 & pearl & tearl \\
\hline & jet & 24 & 33 & 3 & bit & cit \\
\hline & cab & 19 & 15 & 3 & tie & tir \\
\hline & tricycle & 43 & 00 & 8 & lavender & pavenden \\
\hline & canoe & 6 & 08 & 5 & incest & inest \\
\hline & buggy & 5 & 07 & 5 & arson & arsog \\
\hline Bird & robin & 377 & 01 & 5 & cedar & wedar \\
\hline & sparrow & 237 & 01 & 7 & hassock & harrock \\
\hline & cardinal & 208 & 16 & 8 & cucumber & ducumbee \\
\hline & eagle & 161 & 12 & 5 & lying & flind \\
\hline & crow & 149 & 02 & 4 & $\operatorname{tank}$ & mant \\
\hline & finch & 20 & 00 & 5 & aster & raste \\
\hline & ostrich & 17 & 00 & 7 & magenta & mageant \\
\hline & starling & 49 & 01 & 8 & squirrel & squissel \\
\hline & swan & 14 & 04 & 4 & wood & swod \\
\hline & lark & 15 & 04 & 4 & rope & tope \\
\hline Flower & rose & 421 & 18 & 4 & knit & knil \\
\hline & tulip & 209 & 06 & 5 & lemon & lemox \\
\hline & carnation & 183 & 00 & 9 & extortion & exteation \\
\hline & daisy & 176 & 03 & 5 & baker & tader \\
\hline & orchid & 135 & 03 & 6 & endive & encibe \\
\hline & posy & 8 & 00 & 4 & $\operatorname{taxi}$ & tadi \\
\hline & lilac & 45 & 04 & 5 & linen & finen \\
\hline & buttercup & 17 & 00 & 9 & orangeade & orasteade \\
\hline & poppy & 22 & 03 & 5 & zebra & mebra \\
\hline & zinnia & 21 & 00 & 6 & poplar & loplar \\
\hline Weapon & knife & 405 & 86 & 5 & storm & storg \\
\hline & gun & 394 & 142 & 3 & fig & lig \\
\hline & rifle & 163 & 87 & 5 & ruler & rulen \\
\hline & bomb & 122 & 68 & 4 & sled & sler \\
\hline & club & 112 & 178 & 4 & boat & loat \\
\hline & stick & 39 & 42 & 5 & judge & dudge \\
\hline & axe & 34 & 19 & 3 & dew & wep \\
\hline & chain & 27 & 60 & 5 & month & sunth \\
\hline & rock & 23 & 91 & 4 & iris & idis \\
\hline & whip & 27 & 16 & 4 & gown & pown \\
\hline
\end{tabular}




\begin{tabular}{|c|c|c|c|c|c|c|}
\hline \multirow[t]{10}{*}{ Beverage } & milk & 366 & 49 & 4 & lime & lixe \\
\hline & coke & 327 & 03 & 4 & cart & jart \\
\hline & water & 295 & 486 & 5 & satin & salin \\
\hline & coffee & 225 & 78 & 6 & poster & boster \\
\hline & tea & 217 & 29 & 3 & axe & aze \\
\hline & malt & 10 & 01 & 4 & judo & duje \\
\hline & shake & 28 & 03 & 5 & sleet & speet \\
\hline & cocoa & 16 & 03 & 5 & attack & aptak \\
\hline & eggnog & 7 & 00 & 6 & sailor & saitor \\
\hline & pop & 21 & 03 & 3 & hat & hab \\
\hline \multirow[t]{10}{*}{ Tree } & oak & 394 & 16 & 3 & van & nal \\
\hline & maple & 314 & 10 & 5 & orlon & orvon \\
\hline & pine & 214 & 16 & 4 & jade & pade \\
\hline & elm & 210 & 04 & 3 & asp & ars \\
\hline & birch & 134 & 02 & 5 & drill & prill \\
\hline & ash & 45 & 17 & 3 & cop & dop \\
\hline & holly & 11 & 00 & 5 & cycle & kycle \\
\hline & palm & 30 & 30 & 4 & cook & brok \\
\hline & yew & 4 & 00 & 3 & bra & cra \\
\hline & beech & 29 & 06 & 5 & glove & blove \\
\hline \multirow[t]{10}{*}{ Crime } & murder & 387 & 83 & 6 & velvet & pelvet \\
\hline & rape & 271 & 03 & 4 & heat & feap \\
\hline & robbery & 189 & 13 & 7 & apricot & apricom \\
\hline & theft & 171 & 10 & 5 & lathe & sathe \\
\hline & assault & 132 & 18 & 7 & senator & searnot \\
\hline & bigamy & 13 & 00 & 6 & surfing & purfin \\
\hline & libel & 05 & 02 & 5 & camel & lamel \\
\hline & forgery & 22 & 02 & 7 & petunia & setunia \\
\hline & fraud & 21 & 13 & 5 & shack & chack \\
\hline & treason & 22 & 06 & 7 & sequoia & mequoia \\
\hline \multirow[t]{5}{*}{ Weather } & hurricane & 318 & 08 & 9 & cantalope & tantarope \\
\hline & tornado & 303 & 02 & 7 & century & cempury \\
\hline & rain & 297 & 73 & 4 & vise & pise \\
\hline & snow & 266 & 56 & 4 & raft & vaft \\
\hline & hail & 206 & 04 & 4 & pool & rool \\
\hline
\end{tabular}

$\begin{array}{lllll}\text { windstorm } & 14 & 01 & 9 & \text { steamboat steathoat }\end{array}$ $\begin{array}{llllll}\text { drought } & 16 & 08 & 7 & \text { seltzer } & \text { sleartz }\end{array}$ 


\begin{tabular}{|c|c|c|c|c|c|c|}
\hline & mist & 11 & 16 & 4 & hose & sose \\
\hline & smog & 7 & 01 & 4 & zinc & zint \\
\hline & gale & 12 & 02 & 4 & chef & chen \\
\hline \multirow[t]{10}{*}{ Clothing } & shirt & 352 & 29 & 5 & plate & plame \\
\hline & shoes & 274 & 58 & 5 & voice & boite \\
\hline & socks & 330 & 10 & 5 & glass & plast \\
\hline & pants & 318 & 09 & 5 & track & grack \\
\hline & blouse & 261 & 02 & 6 & wrench & brench \\
\hline & boots & 8 & 30 & 5 & stove & shobe \\
\hline & parka & 2 & 00 & 5 & arrow & arrop \\
\hline & apron & 2 & 08 & 5 & egret & egren \\
\hline & smock & 1 & 00 & 5 & jeans & povel \\
\hline & jumper & 13 & 01 & 6 & grocer & trocer \\
\hline \multirow{10}{*}{ Furniture } & chair & 440 & 89 & 5 & tiger & trige \\
\hline & table & 408 & 242 & 5 & torso & porso \\
\hline & bed & 328 & 139 & 3 & jay & jav \\
\hline & sofa & 232 & 09 & 4 & oboe & tobo \\
\hline & desk & 230 & 69 & 4 & cold & lold \\
\hline & chest & 41 & 57 & 5 & flute & drute \\
\hline & bench & 12 & 42 & 5 & dress & dreff \\
\hline & $\cot$ & 2 & 00 & 3 & bat & lat \\
\hline & crib & 3 & 08 & 4 & plum & plub \\
\hline & seat & 2 & 68 & 4 & cart & rart \\
\hline \multirow[t]{10}{*}{ Sport } & football & 396 & 38 & 8 & mushroom & mundroom \\
\hline & baseball & 376 & 62 & 8 & merchant & mercharn \\
\hline & tennis & 329 & 15 & 6 & buffet ruffet & \\
\hline & swimming & 277 & 37 & 8 & daughter & saughtel \\
\hline & soccer & 160 & 03 & 6 & saucer & daucer \\
\hline & softball & 31 & 00 & 8 & sapphire & tapshire \\
\hline & archery & 49 & 01 & 8 & adultery & amultera \\
\hline & boxing & 23 & 00 & 6 & nectar & dectar \\
\hline & handball & 27 & 00 & 8 & strainer & straidet \\
\hline & skiing & 45 & 05 & 6 & cloudy & clouny \\
\hline \multirow[t]{3}{*}{ Vegetable } & carrot & 316 & 05 & 6 & turtle & surtle \\
\hline & peas & 308 & 24 & 4 & swan & twan \\
\hline & corn & 247 & 38 & 4 & fork & sork \\
\hline
\end{tabular}




\begin{tabular}{|c|c|c|c|c|c|}
\hline $\begin{array}{l}\text { beans } \\
\text { potato }\end{array}$ & $\begin{array}{l}237 \\
224\end{array}$ & $\begin{array}{l}13 \\
30\end{array}$ & $\begin{array}{l}5 \\
6\end{array}$ & $\begin{array}{l}\text { steam } \\
\text { maroon }\end{array}$ & $\begin{array}{l}\text { steap } \\
\text { marono }\end{array}$ \\
\hline turnip & 31 & 01 & 6 & squash & squark \\
\hline kale & 20 & 01 & 4 & horn & hort \\
\hline leak & 1 & 05 & 4 & coat & coar \\
\hline onion & 47 & 19 & 5 & clear & cleal \\
\hline radish & 46 & 08 & 6 & locust & pocust \\
\hline
\end{tabular}

Reproduced with permission of the copyright owner. Further reproduction prohibited without permission. 


\section{VITA}

Janice Feagin Del Toro was born in Jacksonville, Florida. She received her Bachelor of Arts Degree in Spanish, and her Master of Arts Degree in Speech from the University of Florida. She began her doctoral studies at the University of Arizona as a predoctoral fellow in the Department of Speech and Hearing Sciences and the National Center for Neurogenic Communication Disorders before transferring to Louisiana State University (LSU). She is currently a faculty member of the Department of Communication Sciences and Disorders at LSU where she teaches graduate courses related to adult communication disorders. She has approximately ten years clinical experience as a speech-language pathologist working with adult neurogenic patients in acute and rehabilitative settings. Her research interests include cognitive communication disorders in adults, aphasia, and psycholinguistics. 


\section{DOCTORAL EXAMINATION AND DISSERTATION REPORT}

Candidate: Janice Feagin Del Toro

Major Field: Communication Disorders

Title of DisBertation: An Examination of Automatic Versus Strategic Semantic Priming Effects in Broca's Aphasia

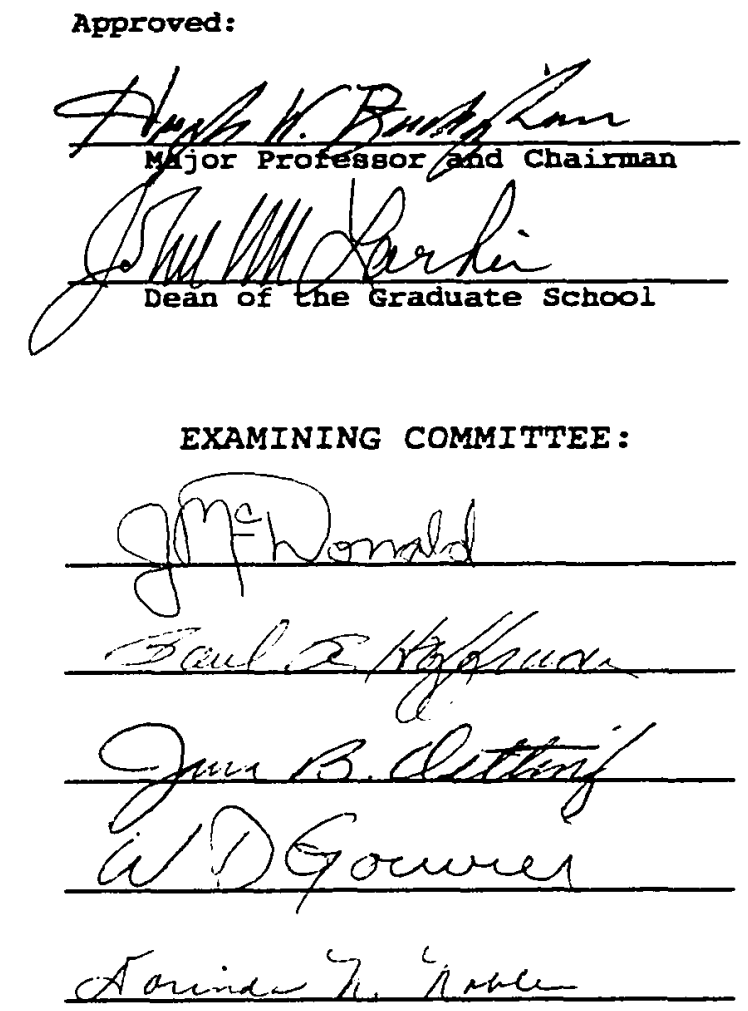

Date of Bxamination:

October 14,1998

Reproduced with permission of the copyright owner. Further reproduction prohibited without permission. 
